\title{
Conformal Nets II: Conformal Blocks
}

\author{
Arthur Bartels $^{1}$, Christopher L. Douglas ${ }^{2} \mathbb{D}$, André Henriques ${ }^{3}$ \\ 1 Mathematisches Institut, Westfälische Wilhelms-Universität Münster, Einsteinstr. 62, 48149 Münster, \\ Germany. E-mail: bartelsa@wwu.de. URL: http://www.math.uni-muenster.de/u/bartelsa \\ 2 Mathematical Institute, Radcliffe Observatory Quarter, Woodstock Road, Oxford, OX2 6GG, UK \\ E-mail: cdouglas@maths.ox.ac.uk. URL: http://people.maths.ox.ac.uk/cdouglas \\ 3 Mathematisch Instituut, Universiteit Utrecht, Postbus 80.010, 3508 TA Utrecht, The Netherlands \\ E-mail: a.g.henriques@uu.nl. URL: http://www.staff.science.uu.nl/henri105
}

Received: 23 October 2016 / Accepted: 31 October 2016

Published online: 9 March 2017 - (C) The Author(s) 2017. This article is an open access publication

\begin{abstract}
Conformal nets provide a mathematical formalism for conformal field theory. Associated to a conformal net with finite index, we give a construction of the 'bundle of conformal blocks', a representation of the mapping class groupoid of closed topological surfaces into the category of finite-dimensional projective Hilbert spaces. We also construct infinite-dimensional spaces of conformal blocks for topological surfaces with smooth boundary. We prove that the conformal blocks satisfy a factorization formula for gluing surfaces along circles, and an analogous formula for gluing surfaces along intervals. We use this interval factorization property to give a new proof of the modularity of the category of representations of a conformal net.
\end{abstract}

\section{Contents}

Introduction . . . . . . . . . . . . . . . . . . . . . . . . . 394

1. Conformal Nets ...................... . . 401

1.A Extending conformal nets to arbitrary 1-manifolds . . . . . . . . . . 402

1.B The algebra associated to a circle . . . . . . . . . . . . . . . 406

2. The Hilbert Space Associated to a Surface . . . . . . . . . . . . . . . . . 408

2.A Canonical up to phase . . . . . . . . . . . . . . . . . . 408

2.B The construction . . . . . . . . . . . . . . . . . . . . . 410

2.C The Hilbert space associated to a disk . . . . . . . . . . . . . . . . . 410

2.D Independence of the cell decomposition . . . . . . . . . . . . . . . . 415

2.E Factorization along circles and along intervals . . . . . . . . . . . . . 424

2.F Conformal blocks . . . . . . . . . . . . . . . . . . . . . . 428

We thank Stefan Stolz and Peter Teichner for their continuous support throughout this project. We would also like to thank Yi-Zhi Huang, Jørgen E. Andersen, and Michael Müger for their help with references. The last author thanks Roberto Longo and Sebastiano Carpi for a pleasant stay in Rome, during which he was able to present this work. 
3. Modularity . . . . . . . . . . . . . . . . . . 431

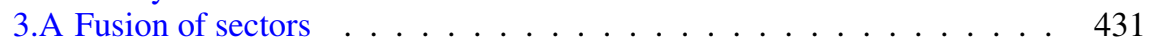

3.B The braiding . . . . . . . . . . . . . . . . . . . 436

3.C Modularity of the category of sectors . . . . . . . . . . . . . . 438

Appendix A. Cyclic Fusion and Fusion Along Graphs . . . . . . . . . . . . . 442

Appendix B. The Hilbert Space Associated to an Annulus . . . . . . . . . . . 444

References . . . . . . . . . . . . . . . . . . . . . 457

\section{Introduction}

Conformal field theory and conformal blocks. Given a $2 \mathrm{~d}$ conformal field theory (CFT), its partition function $Z$ assigns a number to every Riemann surface $\Sigma .{ }^{1}$ The correlation functions generalize the partition function: they assign numbers to Riemann surfaces with marked points (and local coordinates) labelled by fields of the CFT-the fields form a vector space, also called the state space of the conformal field theory.

Conformal blocks were first introduced in the famous paper of Belavin, Polyakov, and Zamolodchikov [11] for the special case of the Virasoro conformal field theory. They are certain holomorphic functions $\mathfrak{F}_{a}$ that enter in a formula for the correlation and partition functions. In its simplest version, the formula reads

$$
Z=\sum h^{a b} \mathfrak{F}_{a} \overline{\mathfrak{F}}_{b}
$$

where $h^{a b}$ is a certain matrix that depends only on the genus of the surface, and on the conformal field theory. These functions $\mathfrak{F}_{a}$ were called conformal blocks "because any correlation function is built up of these functions" [11]. Unlike the correlation and partition functions which are single-valued functions on the moduli space, the conformal blocks typically depend on more data than just the surface and marked points, for instance on a pair-of-pants decomposition of the surface.

It was quickly realized $[22,31]$ that the formula $(0.1)$ can be rewritten in multiple ways (related by what Moore-Seiberg [31] call "duality", that is, the move and that the only object that is truly invariant is the vector space spanned by all the conformal blocks for a given Riemann surface. This is the so-called space of conformal blocks.

The structure of the spaces of conformal blocks. As it turns out, the spaces of conformal blocks only depend on an object that contains somewhat less information (but is more delicate to define) than a conformal field theory. That object goes by the name chiral conformal field theory. To emphasize the difference between chiral conformal field theories and conformal field theories, the latter are also often called full conformal field theories. One may think very roughly of a chiral conformal field theory as being some kind of algebra. For example (unlike for conformal field theories), there exists a notion of representation, also called sector, of a chiral conformal field theory.

The space of conformal blocks associated to a Riemann surface $\Sigma$ is denoted $V(\Sigma)$; the choice of chiral conformal field theory is suppressed from the notation. There is also

\footnotetext{
1 In fact, a non-trivial CFT always has an anomaly and, despite the terminology, the partition function depends not only on a conformal structure on the surface but also on a metric. However, the partition function transforms in a specified way under changes within a given conformal equivalence class, see [22, Sec. 3].
} 
a generalization where the Riemann surface is equipped with a finite collection of points $p_{i}$ (along with first order coordinates at those points), labelled by representations $\lambda_{i}$ of the chiral conformal field theory. The space of conformal blocks is then denoted

$$
V\left(\Sigma, p_{1}, \ldots, p_{n} ; \lambda_{1}, \ldots, \lambda_{n}\right) .
$$

The expected algebraic and differential geometric structures of the spaces of conformal blocks were described in [22] and [31]. They should form holomorphic bundles over the moduli spaces of Riemann surfaces of genus $g$ with $n$ marked points (with first order coordinates). Conformal blocks should certainly be functorial with respect to isomorphisms between Riemann surfaces, but they should also be functorial with respect to orientation reversing isomorphisms [32]: an antiholomorphic isomorphism $f: \Sigma_{1} \rightarrow \Sigma_{2}$ between Riemann surfaces should induce an antiunitary isomorphism

$$
f_{*}: V\left(\Sigma_{1}, p_{1}, \ldots, p_{n} ; \lambda_{1}, \ldots, \lambda_{n}\right) \rightarrow V\left(\Sigma_{2}, f\left(p_{1}\right), \ldots, f\left(p_{n}\right) ; \bar{\lambda}_{1}, \ldots, \bar{\lambda}_{n}\right),
$$

where $\bar{\lambda}$ stands for the representation dual to $\lambda$. These bundles should also be equipped with a hermitian inner product and a projectively flat connection, compatible with both the holomorphic and the unitary structure.

Most importantly, the behavior of these bundles under degeneration should be governed by the so-called factorization formula. Informally, the factorization formula says that

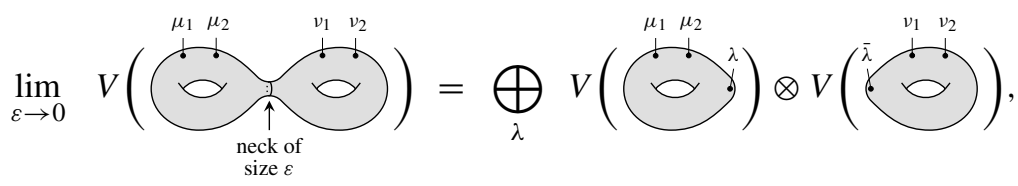

where the direct sum runs over all the irreducible representations $\lambda$ of the chiral conformal field theory.

Constructions of spaces of conformal blocks. If one interprets "chiral conformal field theory" to mean "vertex algebra", then a construction of the spaces of conformal blocks can be found [21, Chapter 10]. However, as far as we know, there is no general proof of, for instance, the factorization property. The literature is somewhat confusing, and we refer the reader to the review paper [25] for a detailed history (restricted to genus zero and one) of what has been proved and when, along with an indication of various false claims in the literature and later retractions.

The first mathematical constructions of the spaces of conformal blocks are due to Tsuchiya-Ueno-Yamada [41] and Hitchin [24], both restricted to the case of WZW models (the WZW models are a certain class of chiral conformal field theories that depend on a choice of compact, simple, connected, simply connected Lie group $G$, and a positive integer $k$ ). An isomorphism between these two constructions was later constructed in [10], [20], and [27], and the projectively flat connections present on both sides were identified in [28]. Factorization for those models was proved in [41].

The unitarity of the spaces of conformal blocks has turned out to be more difficult than the other properties. It is believable that one could combine the unitarity of the modular tensor categories coming from quantum groups ([43], [37, Sec. 4] and references therein), the fact that modular functors are determined by their genus zero data ([3]), and the equivalence of the braided tensor categories associated to quantum groups and to affine Lie algebras ([25, Sec. 3] and references therein) to prove the unitarity of the 
spaces of conformal blocks for most WZW models. However, at the moment, the only statement that is clearly available in the literature is the one for the $S U(n)$ WZW models, due to work of Andersen and Ueno [1,2,4] (see [35] and [30] for an alternative proof for the $S U$ (2) WZW models in genus zero). In an early paper, Axelrod, Della Pietra, and Witten made an attempt at defining the hermitian inner product on the spaces of conformal blocks of the WZW models by writing down a formal expression in terms of functional integrals [5]. They explained how to give a meaning to their formula in genus one, but were unable to deal with the case of higher genus Riemann surfaces.

Formalisms for weak CFT. Positive energy conformal nets [8, Def. 4.5] (which is what people typically mean by the term "conformal net" in the literature, see e.g. [29]) form, along with vertex algebras and Segal's chiral weak CFTs [39, Def. 5.2], one of the three existing mathematical formalizations of the notion of a chiral conformal field theory. (The extent to which these formalisms are equivalent is not completely understood, but note the important advance by Carpi-Kawahigashi-Longo-Weiner constructing a bijection between strongly local unitary vertex algebras and a corresponding class of conformal nets [17].) Given the prominent role that the spaces of conformal blocks play in conformal field theory, it is surprising that nobody has defined them within the formalism of conformal nets. Our paper aims to fill this gap.

The notion of conformal net that we will be using here (introduced in [8], see Definition 1.1 below) is more general than the one commonly used in the literature. Our notion is closely related to that of a conformally covariant net on 2-dimensional Minkowski space [15]. We believe that given such a 2-dimensional net (not necessarily modular invariant), one can obtain a conformal net in our sense by the procedure of time-zero restriction [26, Sec. 4]. It is even plausible that the above procedure provides an equivalence between these two kinds of nets.

Unitary spaces of conformal blocks. As explained above, it is expected that the spaces of conformal blocks form a finite-dimensional vector bundle with hermitian inner product and projectively flat unitary connection over the moduli space of Riemann surfaces. (In the vertex algebra context, this would be under the assumption that the vertex algebra is unitary [19] and subject to appropriate finiteness conditions.) Passing to the associated bundle of projective spaces, the connection becomes genuinely flat and its monodromy yields a projective representation of the fundamental group of the moduli space, a.k.a. the mapping class group.

In the present article, we will be working with conformal nets instead of vertex algebras, and with topological instead of Riemann surfaces. The price to pay for working with topological surfaces instead of Riemann surfaces is that we can only expect the space of conformal blocks $V(\Sigma)$ to form a projective space, not a vector space. (One way to still get a vector space would be to pick a Lagrangian in $H_{1}(\Sigma ; \mathbb{R})$, as explained in [6]. We shall not pursue that approach here.)

We first describe the result of our construction in the case of surfaces without boundary. The object that plays the role of the moduli spaces of Riemann surfaces is the following groupoid. Its objects are connected oriented topological surfaces and its morphisms are isotopy classes of homeomorphisms. We allow orientation reversing maps: the automorphism group of a surface in that groupoid is thus an index two overgroup of the mapping class group. The simplest form of our main construction is:

Theorem 1 (preliminary version: no boundary). Let $\mathcal{A}$ be a conformal net of finite index (Definition 1.15), and let $\Sigma$ be a closed connected oriented topological surface. We can 
associate to $\mathcal{A}$ and $\Sigma$ a finite dimensional projective Hilbert space $\mathbb{P} V(\Sigma)$ called the projective space of conformal blocks of $\Sigma$.

For every isotopy class of homeomorphism $f: \Sigma_{1} \rightarrow \Sigma_{2}$ we have an induced map $f_{*}: \mathbb{P} V\left(\Sigma_{1}\right) \rightarrow \mathbb{P} V\left(\Sigma_{2}\right)$ which is projective unitary if $f$ is orientation preserving and projective antiunitary otherwise. These maps satisfy $(f \circ g)_{*}=f_{*} \circ g_{*}$.

Remark 0.4. Among the conformal nets corresponding to WZW models, only the ones with gauge group $S U(n)$ are known to have finite index (this was proven by $\mathrm{Xu}$ [44], based on the work of Wassermann [42]). For the other WZW conformal nets, the finite index property is also expected to be true but has not yet been proven.

Remark 0.5 . We emphasize that every step of our construction is visibly unitary. This is in stark contrast with the work of Andersen and Ueno [1,2,4], whose proof of unitarity for the $S U(n)$ WZW spaces of conformal blocks relies on a rather non-trivial identification (in [4]) of the $S U(n)$ WZW modular functor with a certain modular functor constructed by Blanchet [14] using skein theory.

Dealing with projective spaces tends to be notationally cumbersome. We therefore prefer to state the above result by saying that for every topological surface $\Sigma$, we have a finite dimensional Hilbert space $V(\Sigma)$ that is "well defined up to a phase" (see Sect. 2.A for a detailed discussion of this notion), and that the maps $f_{*}: V\left(\Sigma_{1}\right) \rightarrow V\left(\Sigma_{2}\right)$ are well defined up to phase.

Conformal nets. In our setup [8], a conformal net is a functor $\mathcal{A}$ from a certain category of intervals (that is, 1 -manifolds diffeomorphic to $[0,1]$ ) to the category of von Neumann algebras. It sends orientation preserving embeddings to homomorphisms and orientation reversing embeddings to antihomomorphisms. We show in Theorem 1.3 that every conformal net admits a canonical extension to the larger category of all compact 1-manifolds. In particular, given a closed 1-manifold $S$ (a disjoint union of circles), there is an associated von Neumann algebra $\mathcal{A}(S)$. If the conformal net has finite index, then $\mathcal{A}(S)$ is a finite direct sum of type $I$ factors. Moreover, if $S^{1}$ is the standard circle then, by Theorem 1.20 , there is a canonical isomorphism

$$
\mathcal{A}\left(S^{1}\right) \cong \bigoplus_{\lambda} \mathbf{B}\left(H_{\lambda}\right)
$$

where $H_{\lambda}$ are the irreducible representations (also called sectors) of the conformal net. (Note that this isomorphism is similar to the decomposition of the reduced universal $C^{*}$-algebra in Carpi-Conti-Heillier-Weiner [16].) For a disjoint union of circles $S=$ $S_{1} \sqcup \cdots \sqcup S_{n}$, we have $\mathcal{A}(S) \cong \mathcal{A}\left(S_{1}\right) \bar{\otimes} \cdots \bar{\otimes} \mathcal{A}\left(S_{n}\right)$. The irreducible summands of $\mathcal{A}(S)$ are indexed by the set of labelings of $\pi_{0}(S)$ by isomorphism classes of irreducible representations of $\mathcal{A}$.

Topological surfaces with smooth boundary. If $\Sigma$ is a compact oriented topological surface with boundary, and $\partial \Sigma$ is equipped with a smooth structure, then our construction produces an infinite dimensional Hilbert-space-well-defined-up-to-phase $V(\Sigma)$, equipped with an action of the von Neumann algebra $\mathcal{A}(\partial \Sigma)$. The relationship between $V(\Sigma)$ and the finite dimensional vector spaces $(0.2)$ is as follows, at least conjecturally. Pick a complex structure on $\Sigma$ and a parametrization of each boundary component by the standard circle. By gluing a copy of the standard disk on each boundary component 
of $\Sigma$, we obtain a closed Riemann surface denoted $\widehat{\Sigma}$. Let $p_{i}$ be the centers of the discs. Then we expect a unitary isomorphism

$$
V(\Sigma) \cong \underset{\begin{array}{c}
\text { labelings } \lambda_{1}, \ldots, \lambda_{m} \\
\text { of } \pi_{0}(\partial \Sigma) \text { by irreps of } \mathcal{A}
\end{array}}{ } V\left(\widehat{\Sigma}, p_{1}, \ldots, p_{n} ; \lambda_{1}, \ldots, \lambda_{n}\right) \otimes H_{\lambda_{1}} \otimes \cdots \otimes H_{\lambda_{n}},
$$

canonical up to phase. Note that the above Hilbert space $V(\Sigma)$ is quite natural from the point of view of conformal field theory. For the chiral WZW models with target $G$, the right hand side of (0.7) is the geometric quantization of the moduli space of holomorphic $G_{\mathbb{C}}$-bundles over $\Sigma$ trivialized over $\partial \Sigma$. That statement was proved by Posthuma [34, eq. just below (3.2)] in the case $G$ is a torus, and its algebro-geometric analog was shown by Teleman [40, Thm. 4] for $G$ semi-simple.

Remark 0.8. If one interprets $V\left(\widehat{\Sigma}, p_{1}, \ldots, p_{n} ; \lambda_{1}, \ldots, \lambda_{n}\right)$ to be the vertex-algebratheoretic spaces of conformal blocks, then the above equation is of course conjectural, as the correspondence between conformal nets and vertex algebras has not yet been worked out to any satisfactory level of detail. On the other hand, if one defines $V\left(\widehat{\Sigma}, p_{1}, \ldots, p_{n} ; \lambda_{1}, \ldots, \lambda_{n}\right)$ via conformal nets, as in $(0.9)$ below, then Eq. $(0.7)$ becomes trivially true.

We can now state our main result for the case of surfaces with boundary. The construction, which is the same as the one for surfaces without boundary, is outlined in Sect. 2.B:

Theorem 1. Let $\mathcal{A}$ be a conformal net with finite index. If $\Sigma$ is a topological surface whose boundary is equipped with a smooth structure, then there is an associated Hilbertspace-well-defined-up-to-phase $V(\Sigma)$ equipped with an action of $\mathcal{A}(\partial \Sigma)$. Each irreducible representation of $\mathcal{A}(\partial \Sigma)$ appears with finite multiplicity in $V(\Sigma)$.

A homeomorphism $f: \Sigma_{1} \rightarrow \Sigma_{2}$ that is smooth on the boundary induces a map, well defined up to phase $f_{*}: V\left(\Sigma_{1}\right) \rightarrow V\left(\Sigma_{2}\right)$ that is unitary if $f$ is orientation preserving and antiunitary otherwise. ${ }^{2}$ These maps are compatible with the algebra actions, and satisfy $(f \circ g)_{*}=f_{*} \circ g_{*}$ up to phase.

Furthermore, if two maps from $\Sigma_{1}$ to $\Sigma_{2}$ are isotopic by an isotopy that is constant on $\partial \Sigma$, then the induced maps from $V\left(\Sigma_{1}\right)$ to $V\left(\Sigma_{2}\right)$ are equal up to phase.

The spaces $V(\Sigma)$ that we assign to a surface $\Sigma$ are constrained by the existence of gluing isomorphisms, discussed below in Theorem 2.

Example. The Hilbert space associated to a pair-of-pants is given by

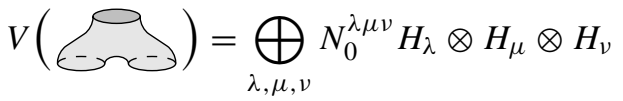

as a representation of $\mathcal{A}(\partial \in \mathcal{C})=\mathcal{A}\left(S^{1}\right) \bar{\otimes} \mathcal{A}\left(S^{1}\right) \bar{\otimes} \mathcal{A}\left(S^{1}\right)$, see Proposition 2.42. Here, the direct sum runs over all triples of irreducible representations, and $N_{0}^{\lambda \mu \nu}$ denotes the multiplicity of the unit object inside the fusion product of $H_{\lambda}, H_{\mu}$, and $H_{\nu}$.

\footnotetext{
2 If $f$ is neither orientation preserving nor orientation reversing (which can happen if the surfaces are disconnected), then $V(f)$ is not defined.
} 
As a consequence of the functoriality of the construction $\Sigma \mapsto V(\Sigma)$, for every surface $\Sigma$ and every conformal net $\mathcal{A}$ with finite index, there is a projective (anti)unitary representations of the following infinite dimensional topological group:

$G(\Sigma):=\left\{f \in\right.$ Homeo $_{+}(\Sigma) \cup$ Homeo- $_{-}(\Sigma):\left.f\right|_{\partial \Sigma}$ is smooth $\} /$ isotopy rel $\partial \Sigma$.

If $\Sigma$ is connected, then the group $G(\Sigma)$ fits into a short exact sequence

$$
1 \rightarrow \Gamma(\Sigma) \rightarrow G(\Sigma) \rightarrow D \rightarrow 1,
$$

where $D=$ Diff $_{+}(\partial \Sigma) \cup$ Diff_- $_{-}(\partial \Sigma)$, and $\Gamma(\Sigma)$ is the mapping class group of $\Sigma$ relative to its boundary. Here, the subscripts $+/-$ refer to orientation preserving/reversing maps, respectively. The above action of $G(\Sigma)$ on $V(\Sigma)$ has already been pointed out by Posthuma in the case of the chiral CFTs associated to lattices [34, Thm. 2.11].

Given the above theorem, we can turn (0.7) around and define the spaces of conformal blocks $V\left(\Sigma ; \lambda_{1}, \ldots, \lambda_{n}\right)$ to be the multiplicity space of $H_{\lambda_{1}} \otimes \cdots \otimes H_{\lambda_{n}}$ in the Hilbert space $V(\Sigma)$, that is

$$
V\left(\Sigma ; \lambda_{1}, \ldots, \lambda_{n}\right):=\operatorname{hom}_{\mathcal{A}(\partial \Sigma)}\left(H_{\lambda_{1}} \otimes \cdots \otimes H_{\lambda_{n}}, V(\Sigma)\right) .
$$

Note that for this definition, $\Sigma$ must have parametrized boundary, since otherwise it is not clear how to let $\mathcal{A}(\partial \Sigma)$ act on $H_{\lambda_{1}} \otimes \cdots \otimes H_{\lambda_{n}}$.

Factorization along circles and along intervals. The most notable property of the spaces of conformal blocks is factorization (0.3):

Theorem 2 (preliminary version: gluing along closed manifolds). Let $\mathcal{A}$ be a conformal net with finite index. Let $\Sigma_{1}$ and $\Sigma_{2}$ be topological surfaces with smooth boundary, and let $S$ be a closed 1-manifold (a disjoint union of circles) equipped with an orientation reversing embedding $S \hookrightarrow \partial \Sigma_{1}$ and an orientation preserving embedding $S \hookrightarrow \partial \Sigma_{2}$. Then there is a unitary isomorphism

$$
V\left(\Sigma_{1} \cup_{S} \Sigma_{2}\right) \cong V\left(\Sigma_{1}\right) \otimes_{\mathcal{A}(S)} V\left(\Sigma_{2}\right)
$$

well defined up to phase and compatible with the actions of $\mathcal{A}\left(\partial \Sigma_{i} \backslash S\right)$.

Moreover, the above isomorphisms satisfy an obvious associativity diagram.

Here is an example illustrating the above result:

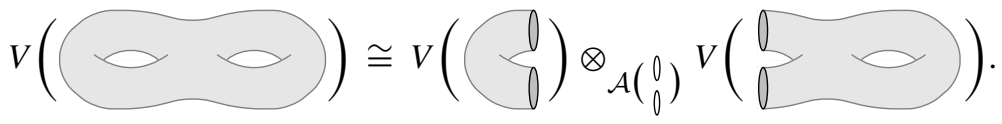

Given the above result, the factorization formula

$$
V\left(\Sigma_{1} \cup_{S} \Sigma_{2} ; \mu_{1}, \ldots \mu_{m}, v_{1}, \ldots v_{n}\right) \cong \bigoplus_{\lambda} V\left(\Sigma_{1} ; \mu_{1}, \ldots \mu_{m}, \lambda\right) \otimes V\left(\Sigma_{2} ; \bar{\lambda}, v_{1}, \ldots v_{n}\right)
$$

follows easily from the definition of the spaces of conformal blocks (0.9) and from the computation (0.6) of the algebra $\mathcal{A}(S)$.

The most novel aspect of our work, impossible to even formulate in the vertex algebraic setup, is a variant of factorization where circles are replaced by intervals. Namely, in the above theorem, we can relax the condition that $S$ be a closed manifold, and allow it to be a manifold with boundary. The algebras associated to 1-manifolds with boundary are no longer type $I$ von Neumann algebras, and so the formulation of our result requires the use of a more elaborate notion of tensor product: the so-called relative tensor product or Connes fusion $([9,13,18,38,42])$, denoted by the symbol $\otimes$. 
Theorem 2. Let $\mathcal{A}$ be a conformal net with finite index. Let $\Sigma_{1}$ and $\Sigma_{2}$ be topological surfaces with smooth boundary. Let $M$ be a compact 1-manifold (possibly disconnected) with boundary, equipped with two embeddings $M \hookrightarrow \partial \Sigma_{1}$ and $M \hookrightarrow \partial \Sigma_{2}$, the first one orientation reversing and the second orientation preserving. Equip the boundary of $\Sigma_{3}:=\Sigma_{1} \cup_{M} \Sigma_{2}$ with a smooth structure such that the smooth structures on $\partial \Sigma_{i}, i \in$ $\{1,2,3\}$ assemble to a "smooth structure" on $\partial \Sigma_{1} \cup_{M} \partial \Sigma_{2}$ in the sense of Definition 1.4.

Then there is a unitary isomorphism

$$
V\left(\Sigma_{1} \cup_{M} \Sigma_{2}\right) \cong V\left(\Sigma_{1}\right) \bigotimes_{\mathcal{A}(M)} V\left(\Sigma_{2}\right),
$$

canonical up to phase and compatible with actions of $\mathcal{A}\left(\partial \Sigma_{1} \backslash \stackrel{\circ}{M}\right)$ and $\mathcal{A}\left(\partial \Sigma_{2} \backslash \stackrel{\circ}{M}\right)$, where $\stackrel{\circ}{M}$ denotes the interior of $M$. Moreover, the above isomorphisms satisfy an obvious notion of associativity.

Finally, the assignment $\Sigma \mapsto V(\Sigma)$ from Theorem 1 is determined by the existence of the gluing law (0.10), and by the requirement that the Hilbert space associated to a disc should be the vacuum sector of $\mathcal{A}$.

For example, the following is an instance of the above isomorphism:

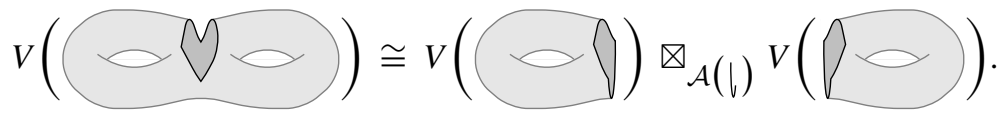

Note that for von Neumann algebras that are direct sums of type $I$ factors, Connes fusion agrees with (the Hilbert space completion of) the usual algebraic tensor product. The previous theorem, about gluing along closed 1-manifolds, is therefore a special case of this last result.

Modularity. In the last section of our paper, we use our technology to revisit some aspects of the representation theory of conformal nets. We reinterpret the monoidal structure on $\operatorname{Rep}(\mathcal{A})$ as the operation of tensoring over $\mathcal{A}(\circlearrowleft \circlearrowleft)$ with the Hilbert space $V\left({ }_{-}\right)$; we also define the braiding of two representations via the action of the homeomorphism $\beta: \Leftrightarrow \rightarrow \in$ that exchanges the two legs of the pair of pants: the braiding of $H$ and $K$ is given by

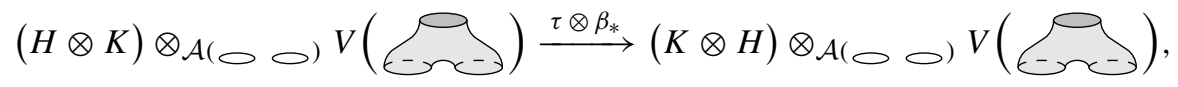

where $\tau$ is the permutation operator.

Finally, we provide an alternative proof of a famous result of Kawahigashi-LongoMüger [26] about the modularity of the representation category of conformal nets:

Theorem. If $\mathcal{A}$ is a conformal net with finite index, then its category of representations is a modular tensor category.

Our proof is based on the property of factorization along intervals. Here, the definition of modularity that we use to verify the above statement is that there are no transparent objects in $\operatorname{Rep}(\mathcal{A})$ aside from the unit object and its multiples (that is, no objects $T$ such that $\nearrow^{T}=Y^{T} /$ for every $X$ in the category). 


\section{Conformal Nets}

In this paper, all 1-manifolds are compact, smooth, and oriented. The standard circle $S^{1}:=\{z \in \mathbb{C}:|z|=1\}$ is the set of complex numbers of modulus one, equipped with the counter-clockwise orientation. By a circle, we shall mean a manifold that is diffeomorphic to the standard circle. Similarly, by an interval, we shall mean a manifold that is diffeomorphic to the standard interval $[0,1]$. For a 1 -manifold $I$, we denote by $\bar{I}$ the same manifold equipped with the opposite orientation. We denote by $\operatorname{Diff}(I)$ the group of diffeomorphisms of $I$, and by $\operatorname{Diff}_{+}(I)$ the subgroup of orientation preserving diffeomorphisms. Given an interval $I$, we also let $\operatorname{Diff}_{0}(I)$ be the group of diffeomorphisms that restrict to the identity near the boundary of $I$. We let INT denote the category whose objects are intervals and whose morphisms are embeddings, not necessarily orientationpreserving.

Let VN be the category whose objects are von Neumann algebras with separable preduals, and whose morphisms are $\mathbb{C}$-linear $*$-homomorphisms, and $\mathbb{C}$-linear $*$ antihomomorphisms. A net is a covariant functor $\mathcal{A}$ : INT $\rightarrow \mathrm{VN}$ taking orientationpreserving embeddings to injective homomorphisms and orientation-reversing embeddings to injective antihomomorphisms. It is said to be continuous if the natural map $\operatorname{Hom}_{\text {INT }}(I, J) \rightarrow \operatorname{Hom}_{\mathrm{VN}}(\mathcal{A}(I), \mathcal{A}(J))$ is continuous for the $\mathcal{C}^{\infty}$ topology on the source and Haagerup's $u$-topology on the target. Given a subinterval $I \subseteq K$, we will often not distinguish between $\mathcal{A}(I)$ and its image in $\mathcal{A}(K)$.

Definition 1.1. A conformal net is a continuous net subject to the following conditions. Here, $I$ and $J$ are subintervals of some interval $K$.

(i) Locality: If $I, J \subset K$ have disjoint interiors, then $\mathcal{A}(I)$ and $\mathcal{A}(J)$ are commuting subalgebras of $\mathcal{A}(K)$.

(ii) Strong additivity: If $K=I \cup J$, then $\mathcal{A}(K)=\mathcal{A}(I) \vee \mathcal{A}(J)$.

(iii) Split property: If $I, J \subset K$ are disjoint, then the map from the algebraic tensor product $\mathcal{A}(I) \otimes_{\text {alg }} \mathcal{A}(J) \rightarrow \mathcal{A}(K)$ extends to the spatial tensor product

$$
\mathcal{A}(I) \bar{\otimes} \mathcal{A}(J) \rightarrow \mathcal{A}(K) .
$$

(iv) Inner covariance: If $\varphi \in \operatorname{Diff}_{0}(I)$, then $\mathcal{A}(\varphi)$ is an inner automorphism.

(v) Vacuum sector: Suppose that $J \subsetneq I$ contains the boundary point $p \in \partial I$. The algebra $\mathcal{A}(J)$ acts on $L^{2}(\mathcal{A}(I))$ via the left action of $\mathcal{A}(I)$, and $\mathcal{A}(\bar{J}) \cong \mathcal{A}(J)^{o p}$ acts on $L^{2}(\mathcal{A}(I))$ via the right action of $\mathcal{A}(I)$. In that case, we require that the action of $\mathcal{A}(J) \otimes_{\text {alg }} \mathcal{A}(\bar{J})$ on $L^{2}(\mathcal{A}(I))$ extends to $\mathcal{A}\left(J \cup_{p} \bar{J}\right)$,

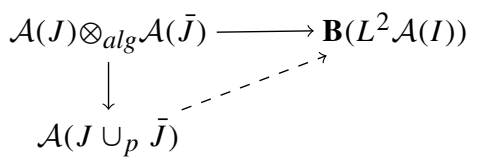

where $J \cup_{p} \bar{J}$ is equipped with any smooth structure that is compatible with the one on $J$, and for which the involution that exchanges $J$ and $\bar{J}$ is smooth. Here, one should picture the interval $J \cup_{p} \bar{J}$ as a submanifold of the double $I \cup_{\partial I} \bar{I}$ of $I$ :

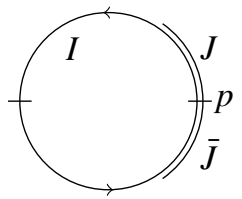


Throughout this paper, we shall assume that our conformal nets are irreducible, i.e., that the algebras $\mathcal{A}(I)$ are factors.

Recall from [8, Section 1.B] that if $S$ is a circle, a Hilbert space is called an $S$-sector of $\mathcal{A}$ if it has compatible actions of $\mathcal{A}(I)$ for every interval $I \subset S$. The category of $S$-sectors

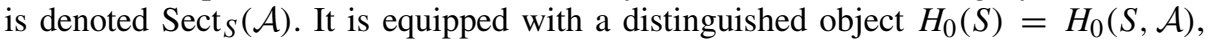
well defined up to non-canonical isomorphism, called the vacuum sector of $\mathcal{A}$ on $S$. The vacuum sector is defined as follows. If $j \in \operatorname{Diff}_{-}(S)$ is an orientation reversing involution that fixes the boundary of some interval $I \subset S$, then we set $H_{0}(S):=L^{2}(\mathcal{A}(I))$. It is equipped with:

- for all $J \subset I$, an action $\mathcal{A}(J) \hookrightarrow \mathcal{A}(I) \stackrel{\text { left action }}{\longrightarrow} \mathbf{B}\left(L^{2}(\mathcal{A}(I))\right)$,

- for all $J \subset j(I)$, an action $\mathcal{A}(J) \hookrightarrow \mathcal{A}(j(I)) \stackrel{\mathcal{A}(j)}{\longrightarrow} \mathcal{A}(I)^{o p} \stackrel{\text { right action }}{\longrightarrow} \mathbf{B}\left(L^{2}(\mathcal{A}(I))\right)$,

and those extend uniquely to the structure of an $S$-sector. When we need to be specific, we will refer to the above construction of $H_{0}(S)$ as the vacuum sector of $\mathcal{A}$ associated to $S, I$, and $j$.

More generally [8, Section 3.C], given a closed 1-manifold $M$, we call a Hilbert space an $M$-sector of $\mathcal{A}$ if it comes with compatible actions of $\mathcal{A}(I)$ for every interval $I \subset M$. The category of $M$-sectors is denoted $\operatorname{Sect}_{M}(\mathcal{A})$.

1.A. Extending conformal nets to arbitrary 1-manifolds. Let $1 \mathrm{MAN}$ be the category whose objects are compact oriented 1-manifolds, possibly disconnected, and whose morphisms are embeddings that are either orientation preserving, or orientation reversing. The goal of this section is to show that every conformal net has a canonical extension to the larger category 1 MAN.

Theorem 1.3. Every conformal net $\mathcal{A}:$ INT $\rightarrow$ VN has a canonical extension $\hat{\mathcal{A}}:$ $1 \mathrm{MAN} \rightarrow \mathrm{VN}$. That extension is symmetric monoidal ${ }^{3}$ in the sense that it takes disjoint unions of 1-manifolds to spatial tensor products of von Neumann algebras.

The proof of this result will occupy this section. We first present some useful technical definitions.

By a $Y$-graph we mean any topological space that is homeomorphic to $\{z \in \mathbb{C}$ : $\left.z^{3} \in[0,1]\right\}$, and by a trivalent graph any compact Hausdorff space that is locally homeomorphic to a $Y$-graph.

Definition 1.4. A smooth structure on a trivalent graph $\Gamma$ is the data of a smooth structure on every interval $I \subset \Gamma$, subject to the conditions:

(i) Whenever $I_{1} \subset I_{2} \subset \Gamma$, the smooth structure on $I_{1}$ is the one inherited from $I_{2}$.

(ii) For every $Y$-graph $Y \subset \Gamma$, there exists a faithful action of the symmetric group $\mathfrak{S}_{3} \rightarrow \operatorname{Homeo}(Y)$, such that for all $g \in \mathfrak{S}_{3}$ and $I \subset Y$, the corresponding map $I \rightarrow g I$ is smooth.

\footnotetext{
3 Note that the symmetric monoidal structure on 1MAN is only partially defined as one cannot take the disjoint union of an orientation preserving map with an orientation reversing one. One way of making precise the sense in which $\hat{\mathcal{A}}$ is symmetric monoidal is to let $B \mathbb{Z} / 2$ be the category with one object and $\mathbb{Z} / 2$ as automorphisms, then note that both $1 \mathrm{MAN}$ and $\mathrm{VN}$ are equipped with a functor to $B \mathbb{Z} / 2$ and that they are symmetric monoidal as categories over $B \mathbb{Z} / 2$. The functor $\hat{\mathcal{A}}$ is then symmetric monoidal over $B \mathbb{Z} / 2$.
} 
Locally around a trivalent point, a smooth structure on $\lambda$ is given by compatible smooth structures on $J, \widehat{ }$, and $L$. The compatibility condition says that there should exist a faithful action of $\mathfrak{S}_{3}$ that induces smooth maps when restricted to each one of the above pieces.

Recall that given a smooth trivalent graph like this: 1 , with circle subgraphs
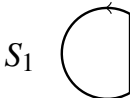

$S_{2}$

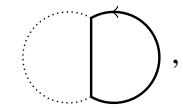

$S_{3}$

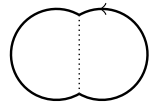

there is a corresponding functor $[8$, Section $1 . \mathrm{C}]$

$$
\bigotimes_{I}: \operatorname{Sect}_{S_{1}}(\mathcal{A}) \times \operatorname{Sect}_{S_{2}}(\mathcal{A}) \rightarrow \operatorname{Sect}_{S_{3}}(\mathcal{A})
$$

given by fusing along $\mathcal{A}(I)$, where $I=S_{1} \cap S_{2}$. Moreover, there is a non-canonical isomorphism of $S_{3}$-sectors

$$
H_{0}\left(S_{1}\right) \bigotimes_{I} H_{0}\left(S_{2}\right) \cong H_{0}\left(S_{3}\right) .
$$

Given a 1-manifold $M$ and a conformal net $\mathcal{A}$, we now describe the algebra $\hat{\mathcal{A}}(M)$. Pick a cover $\mathcal{I}=\left\{I_{1}, \ldots, I_{n}\right\}$ of $M$ consisting of intervals with disjoint interiors. This induces a cover of the 2 -manifold $\Sigma:=M \times[0,1]$ by rectangles $I_{i} \times[0,1]$. Orient the circles $S_{i}:=\partial\left(I_{i} \times[0,1]\right)$ so that their orientation agrees with that of $M \times\{0\}$ on $I_{i} \times\{0\}$. Set $\Gamma:=\bigcup S_{i}$.

$M:$

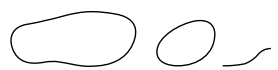

$\Sigma$

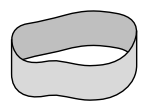

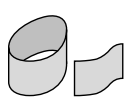
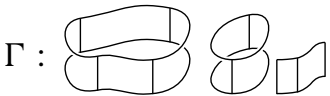

Let us pick local coordinates on $M$ around every point of the finite set $\bigcup_{j} \partial I_{j}$. Using those local coordinates (and the standard coordinates on the copies of $[0,1]$ ), the trivalent graph $\Gamma$ can then be endowed with a smooth structure in the sense of Definition 1.4. In particular, the circles $S_{i}$ have smooth structures and we can talk about their vacuum sectors $H_{0}\left(S_{i}\right)$.

Let $p_{1}, \ldots, p_{m} \in M$ be the points where two of the intervals $I_{i}$ touch each other. If $p_{i} \in I_{j} \cap I_{k}$, then the algebra $A_{i}:=\mathcal{A}\left(\left\{p_{i}\right\} \times[0,1]\right)$ acts on the left on $H_{0}\left(S_{j}\right)$ and on the right on $H_{0}\left(S_{k}\right)$ or the other way around, depending on orientations. We can therefore form the fusion

$$
H_{\Sigma}:=\bigotimes_{\left\{A_{i}\right\}}\left\{H_{0}\left(S_{j}\right)\right\}_{1 \leq i \leq m, 1 \leq j \leq n}
$$

of all the sectors $H_{0}\left(S_{j}\right)$ along all the algebras $A_{i}$, as explained in Definition A.1 in the appendix; this Hilbert space $H_{\Sigma}$ can in fact be well defined up to canonical unitary isomorphism, as established in Theorem B.13, also in the appendix. The result is a $\partial \Sigma$-sector by [8, Cor. 3.31]. We then define

$$
\hat{\mathcal{A}}(M):=\bigvee_{\substack{\text { intervals } \\ I \subset M}} \mathcal{A}(I \times\{0\})
$$

as a subalgebra of $\mathbf{B}\left(H_{\Sigma}\right)$.

In the next two lemmas, we will see that the Hilbert space (1.6) and the algebra (1.7) are independent of the cover $\mathcal{I}$, and of the choice of local coordinates. For the 
moment, we shall write $H_{\Sigma}^{(\mathcal{I})}$ and $\hat{\mathcal{A}}(M)^{(\mathcal{I})}$ to stress the dependence on the cover (the dependence on the local coordinates is also there, but left implicit in the notation). Let us first introduce some terminology to refer to the above structures:

Definition 1.8. A $c$-cover of a compact 1-manifold $M$ consists of a cover $\mathcal{I}=\left\{I_{1}, \ldots, I_{n}\right\}$ by intervals with disjoint interiors, along with germs of local coordinates at every point of the set $\bigcup_{j} \partial I_{j}$.

Given two $c$-covers $\mathcal{I}=\left\{I_{1}, \ldots, I_{n}\right\}$ and $\mathcal{J}=\left\{J_{1}, \ldots, J_{m}\right\}$ of $M$, we shall say that $\mathcal{J}$ refines $\mathcal{I}$, and write it $\mathcal{J} \prec \mathcal{I}$ if the underlying cover $\left\{J_{1}, \ldots, J_{m}\right\}$ refines $\left\{I_{1}, \ldots, I_{n}\right\}$ and the local coordinates of $\mathcal{I}$ agree with those of $\mathcal{J}$ at every point of $\bigcup_{j} \partial I_{j}$.

Lemma 1.9. Let $M$ be a compact 1-manifold equipped with germs of local coordinates at each point of $\partial M$, and let $\mathcal{I}$ and $\mathcal{J}$ be c-covers of $M$ that are compatible with the given local coordinates. Then there is a non-canonical isomorphism of $\partial \Sigma$-sectors

$$
H_{\Sigma}^{(\mathcal{I})} \rightarrow H_{\Sigma}^{(\mathcal{J})}
$$

Proof. We first treat the case when $\mathcal{J}$ refines $\mathcal{I}$. The $c$-cover $\mathcal{J}$ can be obtained from $\mathcal{I}$ by successively subdividing intervals, and so we may as well assume that $\mathcal{I}=$ $\left\{I_{1}, I_{2}, \ldots, I_{n}\right\}$ and $\mathcal{J}=\left\{I_{1}^{\prime}, I_{1}^{\prime \prime}, I_{2}, \ldots, I_{n}\right\}$ with $I_{1}^{\prime} \cup I_{1}^{\prime \prime}=I_{1}$. Let

$$
\begin{gathered}
S_{1}^{\prime}:=\partial\left(I_{1}^{\prime} \times[0,1]\right) \quad S_{1}^{\prime \prime}:=\partial\left(I_{1}^{\prime \prime} \times[0,1]\right) \quad S_{j}:=\partial\left(I_{j} \times[0,1]\right) \\
p_{0}:=I_{1}^{\prime} \cap I_{1}^{\prime \prime} \quad A_{0}:=\mathcal{A}\left(\left\{p_{0}\right\} \times[0,1]\right) .
\end{gathered}
$$

By (1.5), there is an isomorphism of $S_{1}$-sectors

$$
H_{0}\left(S_{1}^{\prime}\right) \bigotimes_{A_{0}} H_{0}\left(S_{1}^{\prime \prime}\right) \cong H_{0}\left(S_{1}\right)
$$

It follows that

$$
\begin{aligned}
H_{\Sigma}^{(\mathcal{J})} & =\bigotimes_{\left\{A_{i}\right\}}\left\{H_{0}\left(S_{1}^{\prime}\right), H_{0}\left(S_{1}^{\prime \prime}\right), H_{0}\left(S_{2}\right), \ldots, H_{0}\left(S_{n}\right)\right\}_{0 \leq i \leq m} \\
& \cong \bigotimes_{\left\{A_{i}\right\}}\left\{H_{0}\left(S_{1}^{\prime}\right) \bigotimes_{A_{0}} H_{0}\left(S_{1}^{\prime \prime}\right), H_{0}\left(S_{2}\right), \ldots, H_{0}\left(S_{n}\right)\right\}_{1 \leq i \leq m} \\
& \cong \bigotimes_{\left\{A_{i}\right\}}\left\{H_{0}\left(S_{1}\right), H_{0}\left(S_{2}\right), \ldots, H_{0}\left(S_{n}\right)\right\}_{1 \leq i \leq m}=H_{\Sigma}^{(\mathcal{I})} .
\end{aligned}
$$

The general case follows from the above special case by the following observation. For any two $c$-covers $\mathcal{I}, \mathcal{I}^{\prime}$ of $M$, there exist $c$-covers $\mathcal{J}, \mathcal{I}^{\prime \prime}, \mathcal{J}^{\prime}$ of $M$ such $\mathcal{I} \succ \mathcal{J} \prec$ $\mathcal{I}^{\prime \prime} \succ \mathcal{J}^{\prime} \prec \mathcal{I}^{\prime}$.

We then have the following result.

Lemma 1.12. Given two c-covers $\mathcal{I}, \mathcal{J}$ of $M$ that induce the same local coordinates at the points of $\partial M$, there is a canonical algebra isomorphism

$$
\hat{\mathcal{A}}(M)^{(\mathcal{I})} \rightarrow \hat{\mathcal{A}}(M)^{(\mathcal{J})}
$$


Proof. Consider the colimit $A:=\operatorname{colim}_{I \subset M} \mathcal{A}(I)$ in the category of $*$-algebras, indexed by the poset of subintervals of $M$. By definition, $\mathcal{A}(M)^{(\mathcal{I})}$ is the von Neumann algebra generated by (the image of) $A$ in $\mathbf{B}\left(H_{\Sigma}^{(\mathcal{I})}\right)$ and, similarly, $\mathcal{A}(M)^{(\mathcal{J})}$ is generated by $A$ in $\mathbf{B}\left(H_{\Sigma}^{(\mathcal{J})}\right)$. By Lemma 1.9 , the Hilbert spaces $H_{\Sigma}^{(\mathcal{I})}$ and $H_{\Sigma}^{(\mathcal{J})}$ are isomorphic as representations of $A$. The two von Neumann algebras are therefore isomorphic, and there is a unique arrow that makes this diagram commute:

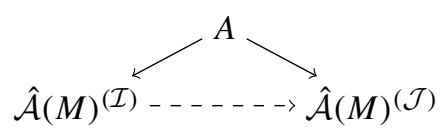

As a corollary, we have:

Corollary 1.13. Given two c-covers $\mathcal{I}$ and $\mathcal{J}$ of $M$ (without any conditions on the local coordinates at the endpoints of $M$ ), there is a canonical algebra isomorphism

$$
\hat{\mathcal{A}}(M)^{(\mathcal{I})} \rightarrow \hat{\mathcal{A}}(M)^{(\mathcal{J})} .
$$

Proof. Write $M$ as a disjoint union of connected component $M=M_{1} \sqcup \cdots \sqcup M_{k}$. We then have canonical isomorphisms $\hat{\mathcal{A}}(M)^{(\mathcal{I})} \cong \hat{\mathcal{A}}\left(M_{1}\right)^{\left(\mathcal{I}_{1}\right)} \bar{\otimes} \ldots \bar{\otimes} \hat{\mathcal{A}}\left(M_{k}\right)^{\left(\mathcal{I}_{k}\right)}$ and $\hat{\mathcal{A}}(M)^{(\mathcal{J})} \cong \hat{\mathcal{A}}\left(M_{1}\right)^{\left(\mathcal{J}_{1}\right)} \bar{\otimes} \cdots \bar{\otimes} \hat{\mathcal{A}}\left(M_{k}\right)^{\left(\mathcal{J}_{k}\right)}$, where $\mathcal{I}_{i}$ and $\mathcal{J}_{i}$ are the restrictions of the $c$-covers $\mathcal{I}$ and $\mathcal{J}$ to the components $M_{i}$ of $M$. It is therefore enough to treat the case when $M$ is connected. If $M$ is a circle, then Lemma 1.12 yields the result. Let us therefore assume that $M$ is an interval.

Let $\mathcal{I}_{0}=\{M\}$ be the $c$-cover consisting of just $M$, along with the local coordinates at $\partial M$ induced by $\mathcal{I}$. Define $\mathcal{J}_{0}$ similarly. Then we have canonical isomorphisms $\hat{\mathcal{A}}(M)^{(\mathcal{I})} \cong \hat{\mathcal{A}}(M)^{\left(\mathcal{I}_{0}\right)} \cong \mathcal{A}(M) \cong \hat{\mathcal{A}}(M)^{\left(\mathcal{J}_{0}\right)} \cong \hat{\mathcal{A}}(M)^{(\mathcal{J})}$.

By the above results, we can see that $\hat{\mathcal{A}}$ defines a functor $1 \mathrm{MAN} \rightarrow \mathrm{VN}$, that its restriction to the subcategory INT recovers $\mathcal{A}$, and that it is symmetric monoidal in the sense that $\hat{\mathcal{A}}(M \sqcup N) \cong \hat{\mathcal{A}}(M) \bar{\otimes} \hat{\mathcal{A}}(N)$. This completes the proof of Theorem 1.3.

Remark 1.14. Given a natural transformation $\tau: \mathcal{A} \rightarrow \mathcal{B}$, it is natural to ask whether it extends to a natural transformation between functors $1 \mathrm{MAN} \rightarrow \mathrm{VN}$. We only know how to prove that it does if the conformal net $\mathcal{A}$ has finite index (Definition 1.15), using Proposition 1.25.

From now on, since there is no risk of confusion, we will drop the hat notation and denote the extension of a conformal net $\mathcal{A}$ to 1 MAN simply by $\mathcal{A}$ :

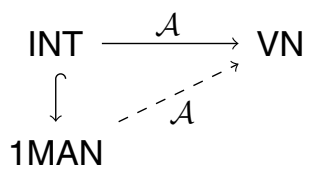


1.B. The algebra associated to a circle. Given a circle $S$, note that an $S$-sector of $\mathcal{A}$ is not the same thing as an $\mathcal{A}(S)$-module. Every $\mathcal{A}(S)$-module admits the structure of an $S$-sector of $\mathcal{A}$, but the converse does not always hold. However, we will see later that for conformal nets with finite index those two notions do agree. The same will also hold for any closed 1-manifold $M$.

Definition 1.15. Let $S$ be a circle, and let $I_{1}, I_{2}, I_{3}, I_{4} \subset S$ be intervals that are arranged so that each $I_{i} \cap I_{i+1}$ (cyclic numbering) is a single point. A conformal net has finite index if the bimodule

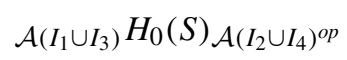

is dualizable.

For the definition of dualizability of bimodules see [7, Sec. 4].

Lemma 1.16. [8, Lem. 3.5] Let $S, I_{1}, I_{2}, \ldots, I_{4}$ be as above, and let $\bar{S}, \bar{I}_{1}, \ldots, \bar{I}_{4}$ be the same manifolds with the reverse orientation. Let $\mathcal{A}$ be a conformal net with finite

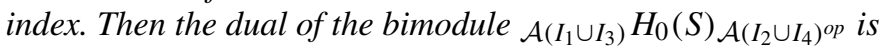

$$
\mathcal{A}\left(\bar{I}_{2} \cup \bar{I}_{4}\right) H_{0}(\bar{S})_{\mathcal{A}\left(\bar{I}_{1} \cup \bar{I}_{3}\right)^{o p}} \text {. }
$$

Here, we have used the canonical identifications $\mathcal{A}\left(\bar{I}_{1} \cup \bar{I}_{3}\right) \cong \mathcal{A}\left(I_{1} \cup I_{3}\right)^{\text {op }}$ and $\mathcal{A}\left(\bar{I}_{2} \cup\right.$ $\left.\bar{I}_{4}\right) \cong \mathcal{A}\left(I_{2} \cup I_{4}\right)^{o p}$.

Let us call a tensor category a fusion category if its underlying linear category is equivalent to $\mathrm{Hilb}^{n}$ (the category whose objects are $n$-tuples of Hilbert spaces) for some finite $n$, and if all its irreducible objects are dualizable. We have seen in [8, Thm. 3.14] that if $\mathcal{A}$ is a conformal net with finite index, then $\operatorname{Sect}_{S}(\mathcal{A})$ is a fusion category. Let $\Delta$ be the set of isomorphism classes of simple sectors, and let $\Delta \rightarrow \Delta: \lambda \mapsto \bar{\lambda}$ be the involution that corresponds to taking the dual sector. Note that the finite set $\Delta$ is independent, up to canonical isomorphism, of the choice of circle $S$.

Lemma 1.17 [8, Lem. 3.17]. Let $S$ be a circle, decomposed into two subintervals $I_{1}$ and $I_{2}$. Then the dual of the bimodule $\mathcal{A}\left(I_{1}\right) H_{\lambda}(S)_{\mathcal{A}\left(I_{2}\right)^{\text {op }}}$ is

$$
\mathcal{A}\left(\bar{I}_{2}\right) H_{\bar{\lambda}}(\bar{S})_{\mathcal{A}\left(\bar{I}_{1}\right)^{o p}}
$$

under the canonical identifications $\mathcal{A}\left(\bar{I}_{1}\right) \cong \mathcal{A}\left(I_{1}\right)^{o p}$ and $\mathcal{A}\left(\bar{I}_{2}\right) \cong \mathcal{A}\left(I_{2}\right)^{o p}$.

For each $\lambda \in \Delta$ and circle $S$, pick a representative $H_{\lambda}(S) \in \operatorname{Sect}_{S}(\mathcal{A})$ of the isomorphism class. Let $\Sigma:=S \times[0,1]$, and let $H_{\Sigma}$ be as in (1.6). Writing $\partial \Sigma$ as $S \sqcup \bar{S}$, then (provided the net $\mathcal{A}$ is finite index) there is a non-canonical unitary isomorphism of $S \sqcup \bar{S}$-sectors [8, Thm. 3.24]

$$
H_{\Sigma} \cong \bigoplus_{\lambda \in \Delta} H_{\lambda}(S) \otimes H_{\bar{\lambda}}(\bar{S})
$$

In fact, this isomorphism can be chosen canonically, as established in Theorem B.17 in the appendix. In light of the next theorem, that isomorphism can be reexpressed as

$$
H_{\Sigma} \cong L^{2} \mathcal{A}(S)
$$


Theorem 1.20. Let $\mathcal{A}$ be a finite irreducible conformal net, and let $S$ be a circle. Then there is a canonical isomorphism

$$
\mathcal{A}(S) \cong \bigoplus_{\lambda \in \Delta} \mathbf{B}\left(H_{\lambda}(S)\right)
$$

Proof. The von Neumann algebra $\mathcal{A}(S)$ is the completion in $\mathbf{B}\left(H_{\Sigma}\right)$ of the algebra generated by all the $\mathcal{A}(I)$ for $I \subset S$. By (1.18), an operator commutes with all those algebras if and only if it is contained in

$$
\bigoplus_{\lambda \in \Delta} \mathbb{C} \otimes \mathbf{B}\left(H_{\lambda}(\bar{S})\right) \subset \mathbf{B}\left(H_{\Sigma}\right)
$$

Taking the commutant, it follows that

$$
\mathcal{A}(S)=\bigvee_{I \subset S} \mathcal{A}(I)=\bigoplus_{\lambda \in \Delta} \mathbf{B}\left(H_{\lambda}(S)\right) \otimes \mathbb{C} \subset \mathbf{B}\left(H_{\Sigma}\right)
$$

The isomorphism (1.18) is a priori non-canonical: it is only well defined up to a phase factor on each direct summand. Those phase factors, however, do not affect the isomorphism (1.21), and the latter is therefore canonical.

Corollary 1.23. If $\mathcal{A}$ is a conformal net with finite index, then the forgetful functor from $\mathcal{A}(S)$-modules to $S$-sectors of $\mathcal{A}$ is an equivalence of categories.

Proof. The functor is clearly fully faithful. To show essential surjectivity, we need to argue that every $S$-sector of $\mathcal{A}$ induces an $\mathcal{A}(S)$-module structure on the same Hilbert space.

Since $\mathcal{A}$ has finite index, the category of $S$-sectors is semisimple [8, Thm. 3.14], and any sector $H$ can be decomposed as

$$
H \cong \bigoplus_{\lambda \in \Delta} H_{\lambda}(S) \otimes M_{\lambda}
$$

where the multiplicity spaces $M_{\lambda}=\operatorname{hom}_{\operatorname{Sect}}(\mathcal{A})\left(H_{\lambda}(S), H\right)$ are Hilbert spaces, and the tensor product is the completed tensor product of Hilbert spaces. It then follows from (1.21) that $H$ is a module for $\mathcal{A}(S)$.

The category of von Neumann algebras is cocomplete. Given a diagram $\left\{A_{i}\right\}$ of von Neumann algebras, its colimit is computed as follows. Let $A_{0}:=\operatorname{colim}_{* \text {-alg }} A_{i}$ be the colimit in the category of $*$-algebras, and let $\mathbb{U}$ be a Grothendieck universe that contains $A_{0}$. Then colim $\mathrm{vN-alg} A_{i}$ is the ultraweak closure of $A_{0}$ inside $\mathbf{B}\left(\bigoplus_{H \in \operatorname{Rep}\left(A_{0}\right) \cap \mathbb{U}} H\right)$, where the direct sum is taken over all representations of $A_{0}$ that are elements of $\mathbb{U}$.

Proposition 1.25. Let $\mathcal{A}$ be a conformal net with finite index and let $S$ be a circle. Then

$$
\mathcal{A}(S)=\underset{I \subset S}{\operatorname{colim}} \mathcal{A}(I)
$$

where the colimit is taken in the category of von Neumann algebras, and is indexed by the poset of subintervals of $S$. 
Proof. Let $A_{0}:=\operatorname{colim}_{*-\text { alg }} \mathcal{A}(I)$, and let $\mathbb{U}$ be a Grothendieck universe that contains $A_{0}$. The Hilbert space $\bigoplus_{H \in \operatorname{Rep}\left(A_{0}\right) \cap \mathbb{U}} H$ is an $S$-sector of $\mathcal{A}$ and is therefore of the form (1.24). The same argument as in the proof of Theorem 1.20 then applies, from which it follows that $A:=\operatorname{colim}_{\mathrm{vN}-\mathrm{alg}} \mathcal{A}(I) \cong \bigoplus_{\lambda \in \Delta} \mathbf{B}\left(H_{\lambda}(S)\right)$, and that the natural map $A \rightarrow \mathcal{A}(S)$ is an isomorphism.

The following result generalizes Corollary 1.23.

Proposition 1.26. If $\mathcal{A}$ is a conformal net with finite index, then the forgetful functor from $\mathcal{A}(M)$-modules to $M$-sectors of $\mathcal{A}$ is an equivalence of categories.

Proof. Let us write $M=S_{1} \sqcup \cdots \sqcup S_{n}$ as a disjoint union of circles, and let $H$ be an $M$-sector. By applying (1.24) to $H$ viewed as an $S_{1}$-sector, we get a decomposition

$$
H \cong \bigoplus_{\lambda \in \Delta} H_{\lambda}\left(S_{1}\right) \otimes K_{\lambda}
$$

The multiplicity spaces $K_{\lambda}$ are equipped with residual actions of the algebras $\mathcal{A}(I)$ for $I \subset S_{2} \sqcup \cdots \sqcup S_{n}$, and are therefore $S_{2} \sqcup \cdots \sqcup S_{n}$-sectors. Applying (1.24) to the $K_{\lambda}$ viewed as $S_{2}$-sectors, we get a further decomposition $H \cong \bigoplus_{\lambda_{1}, \lambda_{2} \in \Delta} H_{\lambda_{1}}\left(S_{1}\right) \otimes$ $H_{\lambda_{2}}\left(S_{2}\right) \otimes K_{\lambda_{1}, \lambda_{2}}$, where $K_{\lambda_{1}, \lambda_{2}}$ are now $S_{3} \sqcup \cdots \sqcup S_{n}$-sectors. After $n$ steps, this yields a decomposition

$$
H \cong \bigoplus_{\lambda_{1}, \lambda_{2}, \ldots, \lambda_{n} \in \Delta} H_{\lambda_{1}}\left(S_{1}\right) \otimes H_{\lambda_{2}}\left(S_{2}\right) \otimes \cdots \otimes H_{\lambda_{n}}\left(S_{n}\right) \otimes K_{\lambda_{1}, \lambda_{2}, \ldots, \lambda_{n}}
$$

where the multiplicity spaces $K_{\lambda_{1}, \lambda_{2}, \ldots, \lambda_{n}}$ are mere Hilbert spaces. The space $H$ is now visibly an $\mathcal{A}\left(S_{1}\right) \bar{\otimes} \ldots \bar{\otimes} \mathcal{A}\left(S_{n}\right)$ module by Theorem 1.20 , that is, an $\mathcal{A}(M)$ module.

\section{The Hilbert Space Associated to a Surface}

2.A. Canonical up to phase. The reader is probably familiar with what it means for a Hilbert space to be well defined up to canonical unitary isomorphism, and what it means for one to be well defined up to non-canonical unitary isomorphism. In this paper, we shall also need an intermediate notion: the notion of a Hilbert space that is well defined up to canonical-up-to-phase unitary isomorphism. An equivalent way of saying that a Hilbert space $H$ is well defined up to canonical-up-to-phase unitary isomorphism, is to say that the associated projective space $\mathbb{P} H$ is well defined up to canonical projective unitary isomorphism. Given that the difference between all those notions is rather subtle, we take the time to spell them out in detail.

One says that a Hilbert space $H$ is well defined up to canonical unitary isomorphism if, even though its construction depends on some choices, there is a given coherent way of identifying all the Hilbert spaces constructed. More precisely, letting $X$ be the set that parametrizes the choices, then for every $x \in X$ there should be a Hilbert space $H_{x}$, for every $x, y \in X$, there should be a given unitary isomorphism $u_{x y}: H_{x} \rightarrow H_{y}$, and for every $x, y, z \in X$, the diagram 


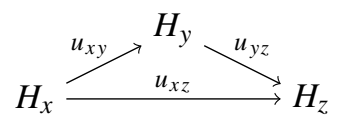

should commute.

By contrast, a Hilbert space $H$ is said to be well defined up to non-canonical (unitary) isomorphism if its construction depends on some choices, any two choices yield isomorphic results, but there are no preferred isomorphisms.

Definition 2.1. We shall say that a Hilbert space $H$ is well defined up to canonical-upto-phase unitary isomorphism if:

- The construction of $H$ depends on some set of choices; say $X$ is the set that parametrizes those choices. For every $x \in X$, we therefore have a Hilbert space $H_{x}$.

- For any two choices $x, x^{\prime} \in X$, there exists a unitary isomorphism $\Phi: H_{x} \rightarrow$ $H_{x^{\prime}}$. Once again, the construction of $\Phi$ depends on some set of choices, let us call $Y\left(x, x^{\prime}\right)$ the set that parametrizes those choices: given $y \in Y\left(x, x^{\prime}\right)$, we have a given isomorphism $\Phi_{y}: H_{x} \rightarrow H_{x^{\prime}}$.

- Given two choices $y, y^{\prime} \in Y\left(x, x^{\prime}\right)$, the isomorphisms $\Phi_{y}$ and $\Phi_{y^{\prime}}$ are equal up to a scalar.

- Finally, for any $y \in Y\left(x, x^{\prime}\right), y^{\prime} \in Y\left(x^{\prime}, x^{\prime \prime}\right), y^{\prime \prime} \in Y\left(x, x^{\prime \prime}\right)$, there exists a scalar $\lambda$ such that $\Phi_{y^{\prime}} \circ \Phi_{y}=\lambda \Phi_{y^{\prime \prime}}$.

Note that the above concepts apply not only to Hilbert spaces, but also to modules over von Neumann algebras, bimodules, sectors, etc. They also apply to functors with values in a category such as Hilb, $A$-modules, etc.

When the mathematical object under consideration is a number, a linear map, or a natural transformation between functors, then the story is somewhat simpler: such an object is either canonical, canonical up to phase, or not canonical (here, "canonical" is a synonym of "well defined").

For example, if $\mathcal{A}$ is an irreducible conformal net, then by Schur's lemma, the vacuum sector $H_{0}(S) \in \operatorname{Sect}_{S}(\mathcal{A})$ is well defined up to canonical-up-to-phase unitary isomorphism. By the same reasoning, the isomorphism $H_{0}\left(S_{1}\right) \bigotimes_{\mathcal{A}(I)} H_{0}\left(S_{2}\right) \cong H_{0}\left(S_{3}\right)$ in (1.5) is canonical up to phase.

In practice, a convenient way of showing that a Hilbert space $H$ is well defined up to canonical-up-to-phase unitary isomorphism is to exhibit a simply connected 2dimensional $\mathrm{CW}$-compex $\mathbb{X}$, and do the following:

- For every vertex $x$ of $\mathbb{X}$, construct a Hilbert space $H_{x}$.

- For every edge $y$ of $\mathbb{X}$ between vertices $x, x^{\prime} \in \mathbb{X}$, construct a unitary isomorphism $\Phi_{y}: H_{x} \rightarrow H_{x^{\prime}}$.

- For every 2-cell of $\mathbb{X}$ with boundary $y_{1} y_{2} \ldots y_{n}$, check that the automorphism $\Phi_{y_{1}} \circ$ $\Phi_{y_{2}} \circ \cdots \circ \Phi_{y_{n}}$ is a scalar.

We call such a CW-complex a definition complex for $H$.

The relation to Definition 2.1 is as follows. The set $X$ is the set of vertices of $\mathbb{X}$. Given $x, x^{\prime} \in X$, the set $Y\left(x, x^{\prime}\right)$ that parametrizes isomorphisms between $H_{x}$ and $H_{x^{\prime}}$ is the set of all sequences of edges in $\mathbb{X}$ that altogether go from $x^{\prime}$ to $x$. The isomorphism $\Phi_{p}: H_{x} \rightarrow H_{x^{\prime}}$ that corresponds to a path $p=y_{1} \cdots y_{n}$ is the composite $\Phi_{y_{1}} \circ \cdots \circ \Phi_{y_{n}}$. In order to check the two conditions in Definition 2.1, we need to show that, given a loop $\gamma$ based at a vertex $x \in \mathbb{X}$, the corresponding automorphism $\Phi_{\gamma} \in \mathrm{U}\left(H_{x}\right)$ is a 
scalar. Because the definition complex is simply connected, one can write $\gamma$ as a product $\prod p_{i} w_{i} p_{i}^{-1}$ where the $p_{i}$ are paths, and the $w_{i}$ are loops along which 2-cells are attached. Since $\Phi_{w_{i}} \in \mathrm{U}(1)$ is central, we conclude that $\Phi_{\gamma}=\prod \Phi_{p_{i}} \Phi_{w_{i}} \Phi_{p_{i}}^{-1}$ is also a scalar, as desired.

2.B. The construction. In this section, we give a construction of a Hilbert space $V(\Sigma) \in$ $\operatorname{Sect}_{\partial \Sigma}(\mathcal{A})$ associated to a topological surface $\Sigma$ with smooth (possibly empty) boundary. The construction depends on the auxiliary choice of a certain kind of cell decomposition of $\Sigma$. We will show later, in Sect. 2.D, that it is actually independent of that choice.

Let us call a cell decomposition regular if for every 2-cell $\mathbb{D}$ the corresponding map $S^{1} \cong \partial \mathbb{D} \rightarrow \Sigma$ is injective, and trivalent if each vertex is incident to exactly three edges. We call a cell decomposition $\Sigma=\mathbb{D}_{1} \cup \cdots \cup \mathbb{D}_{n}$ ordered if the set $\left\{\mathbb{D}_{1}, \ldots, \mathbb{D}_{n}\right\}$ of 2 -cells is ordered. Finally, a cell decomposition is smooth if the 1-skeleton is equipped with a smooth structure in the sense of Definition 1.4, and this smooth structure restricts to the given smooth structure on $\partial \Sigma$. Our construction of $V(\Sigma)$ will depend, a priori, on an ordered regular trivalent smooth cell decomposition of $\Sigma$.

The idea of the construction is to associate to each 2-cell $\mathbb{D} \subset \Sigma$ the vacuum sector $H_{0}(\partial \mathbb{D})$, and to then glue them using Connes fusion.

Let $\Sigma_{i}$ be the 2-manifold $\mathbb{D}_{1} \cup \cdots \cup \mathbb{D}_{i}$, including the case $\Sigma_{0}=\emptyset$. Let us give the 1-manifolds $M_{i}:=\Sigma_{i} \cap \mathbb{D}_{i+1}$ the orientations coming from $\partial \mathbb{D}_{i+1}$ (the orientation of $\partial \mathbb{D}_{i+1}$ is itself induced from that of $\left.\mathbb{D}_{i+1}\right)$. We define $V\left(\Sigma_{i}\right) \in \operatorname{Sect}_{\partial \Sigma_{i}}(\mathcal{A})$ inductively by

$$
\left\{\begin{array}{l}
V(\emptyset):=\mathbb{C} \\
V\left(\Sigma_{i+1}\right):=V\left(\Sigma_{i}\right) \bigotimes_{\mathcal{A}\left(M_{i}\right)} H_{0}\left(\partial \mathbb{D}_{i+1}\right) .
\end{array}\right.
$$

To give a meaning to the above construction, we need to explain how the algebra $\mathcal{A}\left(M_{i}\right)$ acts on the Hilbert spaces $V\left(\Sigma_{i}\right)$ and $H_{0}\left(\partial \mathbb{D}_{i+1}\right)$. The left action on $H_{0}\left(\partial \mathbb{D}_{i+1}\right)$ is obvious when $M_{i} \subsetneq \partial \mathbb{D}_{i+1}$, and follows from Theorem 1.20 for $M_{i}=\partial \mathbb{D}_{i+1}$. Assuming by induction that $V\left(\Sigma_{i}\right)$ is a $\partial \Sigma_{i}$-sector, the right action of $\mathcal{A}\left(M_{i}\right)$ on $V\left(\Sigma_{i}\right)$ follows from Proposition 1.26. To finish the inductive definition, we still need to show that $V\left(\Sigma_{i+1}\right)$ is a $\partial \Sigma_{i+1}$-sector. This is obvious for $M_{i}=\partial \mathbb{D}_{i+1}$, and follows from [8, Lem. 3.30] for $M_{i} \subsetneq \partial \mathbb{D}_{i+1}$.

2.C. The Hilbert space associated to a disk. The goal of this section is to show that if $\Sigma$ is a disk, then regardless of the choice of cell decomposition of $\Sigma$, the resulting sector $V(\Sigma) \in \operatorname{Sect}_{\partial \Sigma}(\mathcal{A})$ is the vacuum $H_{0}(\partial \Sigma)$.

In order to compute the sector associated to a disc, we will need to address the seemingly more difficult problem of computing the isomorphism type of the sector associated to a sphere with $n$ holes. Given $H_{\lambda_{1}}, \ldots, H_{\lambda_{n}} \in \operatorname{Sect}(\mathcal{A})$, let $N_{0}^{\lambda_{1}, \ldots, \lambda_{n}}$ denote the multiplicity of $H_{0}$ inside $H_{\lambda_{1}} \otimes \cdots \otimes H_{\lambda_{n}}$.

Recall from [8, Sec. 1B] that given a sector $H_{\lambda} \in \operatorname{Sect}(\mathcal{A})$ and given a circle $S$, we write $H_{\lambda}(S) \in \operatorname{Sect}_{S}(\mathcal{A})$ for the associated $S$-sector of $\mathcal{A}$. It is given by $\varphi^{*} H_{\lambda}$ for some $\varphi \in \operatorname{Diff}_{+}\left(S, S^{1}\right)$, and is only well defined up to non-canonical isomorphism.

Lemma 2.3. Let $\mathcal{A}$ be a conformal net with finite index and let $\Sigma$ be a genus zero surface (a sphere with holes). Equip $\Sigma$ with an ordered regular trivalent smooth cell decomposition $\Sigma=\mathbb{D}_{1} \cup \cdots \cup \mathbb{D}_{n}$ and let $V(\Sigma)$ be the corresponding Hilbert space. 
Decompose the boundary of $\Sigma$ as a union of circles $\partial \Sigma=S_{1} \cup \cdots \cup S_{m}$. Then for every choice of $\lambda_{1}, \ldots, \lambda_{m} \in \Delta$, the vector space

$$
\operatorname{hom}_{\operatorname{Sect}}{ }_{\partial \Sigma}(\mathcal{A})\left(H_{\lambda_{1}}\left(S_{1}\right) \otimes \cdots \otimes H_{\lambda_{m}}\left(S_{m}\right), V(\Sigma)\right)
$$

has dimension $N_{0}^{\lambda_{1}, \ldots, \lambda_{m}}$.

Proof. Letting $\Sigma^{\prime}:=\mathbb{D}_{1} \cup \cdots \cup \mathbb{D}_{n-1}$ and $M:=\partial \mathbb{D}_{n} \cap \Sigma^{\prime}$, we have by definition

$$
V(\Sigma)=V\left(\Sigma^{\prime}\right) \bigotimes_{\mathcal{A}(M)} H_{0}\left(\partial \mathbb{D}_{n}\right)
$$

The 1-manifold $M$ is either a circle or a union of intervals. If $M$ is a circle, then $\Sigma^{\prime}$ is connected and we can write its boundary $\partial \Sigma^{\prime}$ as $S_{0} \cup S_{1} \cup \cdots \cup S_{m}$ with $M=\bar{S}_{0}$. Using that for any right $\mathcal{A}(M)$-module $K$, there is a canonical isomorphism

$$
K \otimes_{\mathcal{A}(M)} H_{0}(M) \cong \operatorname{hom}_{\mathcal{A}\left(S_{0}\right)}\left(H_{0}\left(S_{0}\right), K\right),
$$

we see by induction on $n$ that

$$
\begin{aligned}
& \operatorname{hom}_{\operatorname{Sect}_{\partial \Sigma}(\mathcal{A})}\left(H_{\lambda_{1}}\left(S_{1}\right) \otimes \cdots \otimes H_{\lambda_{m}}\left(S_{m}\right), V(\Sigma)\right) \\
& \cong \operatorname{hom}_{\operatorname{Sect}_{\partial \Sigma}(\mathcal{A})}\left(H_{\lambda_{1}}\left(S_{1}\right) \otimes \cdots \otimes H_{\lambda_{m}}\left(S_{m}\right), V\left(\Sigma^{\prime}\right) \otimes_{\mathcal{A}(M)} H_{0}(M)\right) \\
& \cong \operatorname{hom}_{\operatorname{Sect}_{\partial \Sigma^{\prime}}(\mathcal{A})}\left(H_{0}\left(S_{0}\right) \otimes H_{\lambda_{1}}\left(S_{1}\right) \otimes \cdots \otimes H_{\lambda_{m}}\left(S_{m}\right), V\left(\Sigma^{\prime}\right)\right)
\end{aligned}
$$

has dimension $N_{0}^{0, \lambda_{1}, \ldots, \lambda_{m}}=N_{0}^{\lambda_{1}, \ldots, \lambda_{m}}$, as desired.

We now assume that $M$ is a union of intervals. Decompose $\Sigma^{\prime}$ as a disjoint union of connected surfaces $\Sigma^{\prime}=\Sigma_{1}^{\prime} \cup \cdots \cup \Sigma_{l}^{\prime}$, and write $\partial \Sigma_{i}^{\prime}=S_{i 1}^{\prime} \cup S_{i 2}^{\prime} \cup \cdots \cup S_{i k_{i}}^{\prime}$. Up to homeomorphism, the surface $\Sigma$ appears as follows:

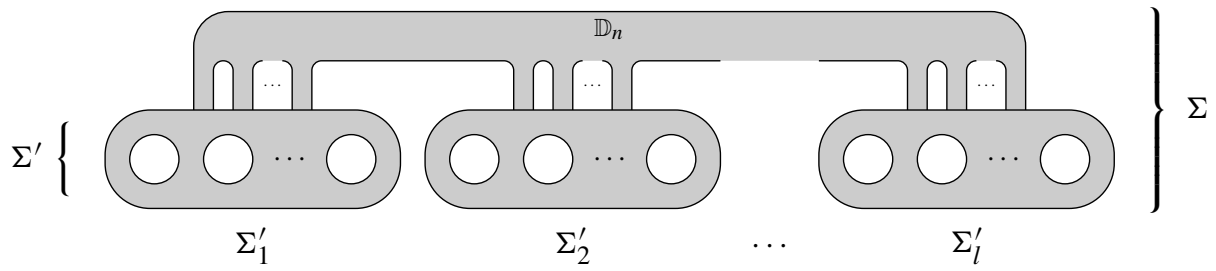

Indeed, since $\Sigma$ has genus zero, the manifold $M$ can only intersect one boundary component of each $\Sigma_{i}^{\prime}$. By renumbering the components of $\partial \Sigma_{i}^{\prime}$, we may assume that $M \cap S_{i 1}^{\prime} \neq \emptyset$ and $M \cap S_{i j}^{\prime}=\emptyset$ for $j \geq 2$.

Recall that we are trying to show that

$$
\operatorname{dim} \operatorname{hom}_{\operatorname{Sect}_{\partial \Sigma}(\mathcal{A})}\left(H_{\lambda_{1}}\left(S_{1}\right) \otimes \cdots \otimes H_{\lambda_{m}}\left(S_{m}\right), V(\Sigma)\right)=N_{0}^{\lambda_{1}, \ldots, \lambda_{m}} .
$$

By induction on $n$, we know that for every $i$

$$
\operatorname{dim} \operatorname{hom}_{\operatorname{Sect}_{\partial \Sigma_{i}^{\prime}}(\mathcal{A})}\left(H_{\lambda_{i 1}}\left(S_{i 1}^{\prime}\right) \otimes \cdots \otimes H_{\lambda_{i k_{i}}}\left(S_{i k_{i}}^{\prime}\right), V\left(\Sigma_{i}^{\prime}\right)\right)=N_{0}^{\lambda_{i 1}, \ldots, \lambda_{i k_{i}}}
$$

Let $b_{i}$ be the number of connected components of $\partial \mathbb{D}_{n} \cap \Sigma_{i}^{\prime}$. Before treating the general case, we prove it for certain small values of $l$ and $b_{1}, \ldots, b_{l}$. 
If $l=1$ and $b_{1}=2$ then, up to homeomorphism, $\Sigma=\Sigma^{\prime} \cup \mathbb{D}_{n}$ appears as follows:

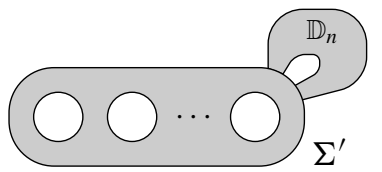

By Lemma 2.8 below and induction on $n, V\left(\Sigma^{\prime}\right)$ is isomorphic to the $\partial \Sigma^{\prime}$-sector associated to this cellular decomposition: 1 . It follows that $V(\Sigma)$ is isomorphic to the $\partial \Sigma$-sector associated to the following decomposition of $\Sigma$ :

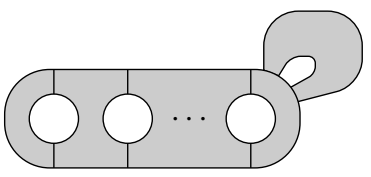

That decomposition is of the form covered by Lemma 2.8, and so (2.5) follows.

We now treat the case $l=2, b_{1}=b_{2}=1$, where $\Sigma$ appears as follows:

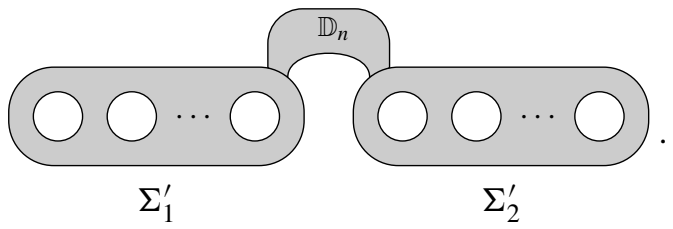

Let $M_{1}=\partial \mathbb{D}_{n} \cap \Sigma_{1}^{\prime}$ and $M_{2}=\partial \mathbb{D}_{n} \cap \Sigma_{2}^{\prime}$. Once again, by Lemma 2.8 and induction on $n$, we know that $V\left(\Sigma_{i}^{\prime}\right)$ is isomorphic to the $\partial \Sigma_{i}^{\prime}$-sector constructed from the cellular decomposition $\bigcirc \cdots$. It follows that

$$
V(\Sigma)=\left(V\left(\Sigma_{1}^{\prime}\right) \otimes V\left(\Sigma_{2}^{\prime}\right)\right) \underset{\mathcal{A}(M)}{\otimes} H_{0}\left(\partial \mathbb{D}_{n}\right) \cong V\left(\Sigma_{1}^{\prime}\right) \underset{\mathcal{A}\left(\bar{M}_{1}\right)}{\otimes} H_{0}\left(\partial \mathbb{D}_{n}\right) \underset{\mathcal{A}\left(M_{2}\right)}{\otimes} V\left(\Sigma_{2}^{\prime}\right)
$$

is isomorphic to the $\partial \Sigma$-sector associated to the following decomposition of $\Sigma$ :

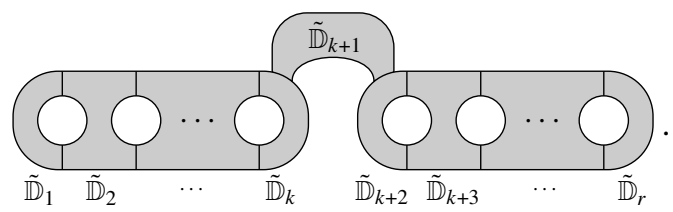

As $H_{0}\left(\partial \tilde{\mathbb{D}}_{k}\right) \bigotimes_{\mathcal{A}\left(\bar{M}_{1}\right)} H_{0}\left(\partial \tilde{\mathbb{D}}_{k+1}\right) \bigotimes_{\mathcal{A}\left(M_{2}\right)} H_{0}\left(\partial \tilde{\mathbb{D}}_{k}\right) \cong H_{0}\left(\partial\left(\mathbb{D}_{k} \cup \mathbb{D}_{k+1} \cup \mathbb{D}_{k+2}\right)\right)$, the sector $V(\Sigma)$ is also isomorphic to the one associated to the following decomposition:

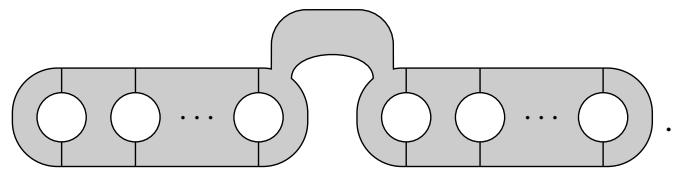

That decomposition is of the form covered by Lemma 2.8, and (2.5) follows. 
We now treat the general case. We assume by induction that $V\left(\Sigma^{\prime}\right)$ satisfies (2.5). Consider the refinement $\Sigma=\tilde{\mathbb{D}}_{1} \cup \ldots \cup \tilde{\mathbb{D}}_{s}$ of (2.4) given by $\tilde{\mathbb{D}}_{i}:=\mathbb{D}_{i}$ for $i<n$, and where $\mathbb{D}_{n}$ is subdivided into cells $\tilde{\mathbb{D}}_{n}, \tilde{\mathbb{D}}_{n+1}, \ldots, \tilde{\mathbb{D}}_{s}$ as follows:

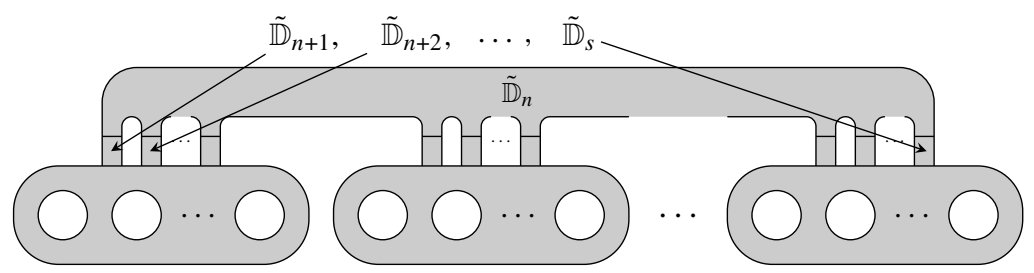

Recall that by definition, the sector $V(\Sigma)$ is associated to the original cell decomposition $\Sigma=\mathbb{D}_{1} \cup \ldots \cup \mathbb{D}_{n}$. Letting $I_{i}:=\partial \tilde{\mathbb{D}}_{n+i} \cap \tilde{\mathbb{D}}_{n}$ and $M_{i}:=\partial \tilde{\mathbb{D}}_{n+i} \cap \Sigma^{\prime}$, we see that

$$
\begin{aligned}
V(\Sigma) & =V\left(\Sigma^{\prime}\right) \bigotimes_{\mathcal{A}(M)} H_{0}\left(\partial \mathbb{D}_{n}\right) \\
& =V\left(\Sigma^{\prime}\right) \bigotimes_{\mathcal{A}\left(M_{1} \cup \ldots \cup M_{s}\right)} H_{0}\left(\partial \mathbb{D}_{n}\right) \\
& \cong V\left(\Sigma^{\prime}\right) \bigotimes_{\mathcal{A}\left(M_{1} \cup \ldots \cup M_{s}\right)}\left(H_{0}\left(\partial \tilde{\mathbb{D}}_{n}\right) \bigotimes_{\mathcal{A}\left(I_{1} \cup \ldots \cup I_{s}\right)} \bigotimes_{i \geq 1} H_{0}\left(\partial \tilde{\mathbb{D}}_{n+i}\right)\right) \\
& \cong\left(\left(V\left(\Sigma^{\prime}\right) \otimes H_{0}\left(\partial \tilde{\mathbb{D}}_{n}\right)\right) \bigotimes_{\mathcal{A}\left(M_{1} \cup I_{1}\right)} H_{0}\left(\partial \tilde{\mathbb{D}}_{n+1}\right)\right) \cdots \bigotimes_{\mathcal{A}\left(M_{s} \cup I_{s}\right)} H_{0}\left(\partial \tilde{\mathbb{D}}_{n+s}\right)
\end{aligned}
$$

is isomorphic to the sector associated to the refined cell decomposition $\tilde{\mathbb{D}}_{1} \cup \cdots \cup \tilde{\mathbb{D}}_{s}$ of $\Sigma$.

For each $i \leq s$, let $\tilde{\Sigma}_{i}:=\tilde{\mathbb{D}}_{1} \cup \ldots \cup \tilde{\mathbb{D}}_{i}$. Recall that by assumption $V\left(\tilde{\Sigma}_{n-1}\right)=V\left(\Sigma^{\prime}\right)$ satisfies the Eq. (2.5). Clearly, $V\left(\tilde{\Sigma}_{n}\right) \cong V\left(\tilde{\Sigma}_{n-1}\right) \otimes H_{0}\left(\partial \tilde{\mathbb{D}}_{n}\right)$ then also satisfies (2.5). We now proceed by induction on $i$, and assume that $V\left(\tilde{\Sigma}_{i-1}\right)$ satisfies (2.5) for some $i \leq s$. By construction, the intersection $\partial \tilde{\mathbb{D}}_{i} \cap \tilde{\Sigma}_{i-1}=M_{i} \cup I_{i}$ is the union of exactly two intervals. If these two intervals belong to the same connected component of $\tilde{\Sigma}_{i-1}$, then the situation is as in (2.6); if they belong to different connected component of $\tilde{\Sigma}_{i-1}$, then the situation is as in (2.7). In both cases, we can apply our intermediate results and conclude that $V\left(\tilde{\Sigma}_{i}\right)$ satisfies $(2.5)$.

The following lemma was used in the previous proof:

Lemma 2.8. Let $\Sigma$ be a sphere with $n$ holes. Equip its boundary components $S_{1}, S_{2}, \ldots$, $S_{n}$ with the orientation induced from $\Sigma$. Let $\mathcal{A}$ be a conformal net with finite index, and let $V(\Sigma)$ be the Hilbert space constructed from the following cellular decomposition of $\Sigma$ :

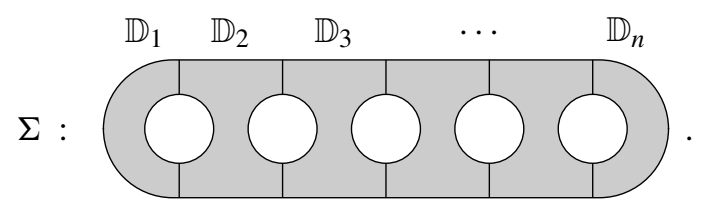

Then the vector space

$$
\operatorname{hom}_{\operatorname{Sect}_{\partial \Sigma}(\mathcal{A})}\left(H_{\lambda_{1}}\left(S_{1}\right) \otimes \cdots \otimes H_{\lambda_{n}}\left(S_{n}\right), V(\Sigma)\right)
$$

has dimension $N_{0}^{\lambda_{1}, \ldots, \lambda_{n}}$. 
Proof. Let us number the boundary components of $\Sigma$ as follows:

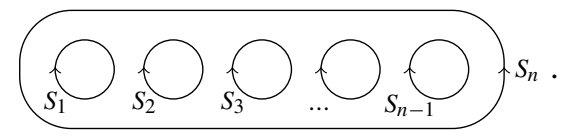

Let $\Sigma^{\prime}:=\mathbb{D}_{1} \cup \cdots \cup \mathbb{D}_{n-1}$, and let $S^{\prime}$ be the component of $\partial \Sigma^{\prime}$ that touches $\mathbb{D}_{n}$. Let also $J_{1}:=S^{\prime} \cap S_{n-1}$ and $J_{2}:=S^{\prime} \cap S_{n}$ :
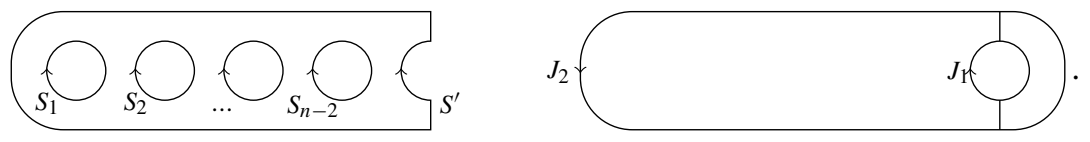

By induction we may assume that

$$
\operatorname{dim} \operatorname{hom}\left(H_{\lambda_{1}}\left(S_{1}\right) \otimes \cdots \otimes H_{\lambda_{n-2}}\left(S_{n-2}\right) \otimes H_{v}\left(S^{\prime}\right), V\left(\Sigma^{\prime}\right)\right)=N_{0}^{\lambda_{1}, \ldots, \lambda_{n-2}, v} .
$$

Applying (1.24) with respect to the circle $S^{\prime}$ we obtain a decomposition

$$
V\left(\Sigma^{\prime}\right) \cong \bigoplus_{\nu \in \Delta} M_{\nu} \otimes H_{\nu}\left(S^{\prime}\right)
$$

where the $M_{v}$ are $S_{1} \sqcup \cdots \sqcup S_{n-2}$-sectors. Thus

$$
\begin{aligned}
\operatorname{hom}\left(H_{\lambda_{1}}\left(S_{1}\right) \otimes \ldots \otimes H_{\lambda_{n-2}}\right. & \left.\left(S_{n-2}\right), M_{v}\right) \\
& \cong \operatorname{hom}\left(H_{\lambda_{1}}\left(S_{1}\right) \otimes \ldots \otimes H_{\lambda_{n-2}}\left(S_{n-2}\right) \otimes H_{v}\left(S^{\prime}\right), V\left(\Sigma^{\prime}\right)\right)
\end{aligned}
$$

is of dimension $N_{0}^{\lambda_{1}, \ldots, \lambda_{n-2}, v}$. Let $M:=\mathbb{D}_{n-1} \cap \mathbb{D}_{n}$, so that $V(\Sigma)=V\left(\Sigma^{\prime}\right) \bigotimes_{\mathcal{A}(M)}$ $H_{0}\left(\partial \mathbb{D}_{n}\right)$. We then have

$$
\begin{aligned}
\operatorname{hom} & \left(H_{\lambda_{1}}\left(S_{1}\right) \otimes \cdots \otimes H_{\lambda_{n}}\left(S_{n}\right), V(\Sigma)\right) \\
\cong & \operatorname{hom}\left(H_{\lambda_{1}}\left(S_{1}\right) \otimes \cdots \otimes H_{\lambda_{n}}\left(S_{n}\right), V\left(\Sigma^{\prime}\right) \otimes_{\mathcal{A}(M)} H_{0}\left(\partial \mathbb{D}_{n}\right)\right) \\
\cong & \operatorname{hom}\left(H_{\lambda_{1}}\left(S_{1}\right) \otimes \cdots \otimes H_{\lambda_{n}}\left(S_{n}\right), \bigoplus_{\nu \in \Delta} M_{\nu} \otimes H_{\nu}\left(S^{\prime}\right) \otimes_{\mathcal{A}(M)} H_{0}\left(\partial \mathbb{D}_{n}\right)\right) \\
\cong & \bigoplus_{v \in \Delta} N_{0}^{\lambda_{1}, \ldots, \lambda_{n-2}, \nu} \operatorname{hom}\left(H_{\lambda_{n-1}}\left(S_{n-1}\right) \otimes H_{\lambda_{n}}\left(S_{n}\right), H_{\nu}\left(S^{\prime}\right) \otimes_{\mathcal{A}(M)} H_{0}\left(\partial \mathbb{D}_{n}\right)\right) .
\end{aligned}
$$

By Frobenius reciprocity,

$$
\begin{aligned}
& \operatorname{hom}\left(H_{\lambda_{n-1}}\left(S_{n-1}\right) \otimes H_{\lambda_{n}}\left(S_{n}\right), H_{v}\left(S^{\prime}\right) \bigotimes_{\mathcal{A}(M)} H_{0}\left(\partial \mathbb{D}_{n}\right)\right) \\
& \cong \operatorname{hom}\left(\overline{H_{0}\left(\partial \mathbb{D}_{n}\right)},\left(\overline{H_{\lambda_{n-1}}\left(S_{n-1}\right)} \otimes \overline{H_{\lambda_{n}}\left(S_{n}\right)}\right) \bigotimes_{\mathcal{A}\left(J_{1}\right) \bar{\otimes} \mathcal{A}\left(J_{2}\right)} H_{v}\left(S^{\prime}\right)\right) \\
& \cong \operatorname{hom}\left(\overline{H_{0}\left(\partial \mathbb{D}_{n}\right)}, \overline{H_{\lambda_{n-1}}\left(S_{n-1}\right)} \bigotimes_{\mathcal{A}\left(J_{1}\right)} H_{v}\left(S^{\prime}\right) \bigotimes_{\mathcal{A}\left(\bar{J}_{2}\right)} \overline{H_{\lambda_{n}}\left(S_{n}\right)}\right) \\
& \cong \operatorname{hom}\left(H_{0}\left(\partial \overline{\mathbb{D}}_{n}\right), H_{\bar{\lambda}_{n-1}}\left(\bar{S}_{n-1}\right) \bigotimes_{\mathcal{A}\left(J_{1}\right)} H_{v}\left(S^{\prime}\right) \bigotimes_{\mathcal{A}\left(\bar{J}_{2}\right)} H_{\bar{\lambda}_{n}}\left(\bar{S}_{n}\right)\right)
\end{aligned}
$$

is of dimension $N_{0}^{\bar{\lambda}_{n-1}, v, \bar{\lambda}_{n}}=N_{0}^{\bar{\nu}, \lambda_{n-1}, \lambda_{n}}$, where we have used Lemmas 1.16 and 1.17 in order to identify the duals of $H_{0}\left(\partial \mathbb{D}_{n}\right), H_{\lambda_{n-1}}\left(S_{n-1}\right)$, and $H_{\lambda_{n}}\left(S_{n}\right)$. It follows that $\operatorname{hom}\left(H_{\lambda_{1}}\left(S_{1}\right) \otimes \cdots \otimes H_{\lambda_{n}}\left(S_{n}\right), V(\Sigma)\right)$ has dimension

$$
\sum_{v \in \Delta} N_{0}^{\lambda_{1}, \ldots, \lambda_{n-2}, v} N_{0}^{\bar{v}, \lambda_{n-1}, \lambda_{n}}=N_{0}^{\lambda_{1}, \ldots, \lambda_{n-1}, \lambda_{n}}
$$


Note that while Lemma 2.3 pins down the isomorphism type of $V(\Sigma)$, it does not say anything about how well defined the Hilbert space is. If the surface $\Sigma$ is a disk, then we can get a little bit more:

Corollary 2.9. Let $\mathcal{A}$ be a conformal net with finite index and let $\mathbb{D}$ be a disc. Then the Hilbert space $V(\mathbb{D})$ associated to an ordered regular trivalent smooth cell decomposition of $\mathbb{D}$ is given by the vacuum sector $H_{0}(\partial \mathbb{D})$. It is independent of the cell decomposition, and well defined up to canonical-up-to-phase unitary isomorphism.

Proof. The isomorphism $V(\mathbb{D}) \cong H_{0}(\partial \mathbb{D})$ is immediate from Lemma 2.3. Moreover, by Schur's lemma, since $V(\mathbb{D})$ is irreducible, it is well defined up to canonical-up-to-phase unitary isomorphism.

2.D. Independence of the cell decomposition. We have seen in (2.2) that it is possible to define a sector $V(\Sigma) \in \operatorname{Sect}_{\partial \Sigma}(\mathcal{A})$ for every ordered regular trivalent smooth cell decomposition of $\Sigma$. For technical reasons, it is convenient to replace the ordering of the set of 2-cells by a slightly different structure:

Definition 2.10. Let $\Sigma$ be a topological surface with smooth boundary. A soccer ball decomposition is a regular trivalent smooth cell decomposition (see Sect. 2.B), equipped with a partition of the set of 2-cells into subsets $X_{\text {white }}$ and $X_{\text {black }}$, such that every interior vertex is adjacent to two white cells and one back cell, and every boundary vertex is adjacent to two white cells.

We illustrate the above definition with an example:

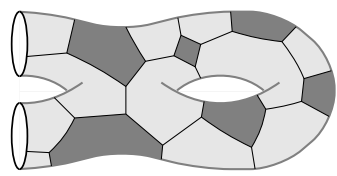

Such decompositions exist in great abundance: starting with any regular cell decomposition, we can get a soccer ball decomposition by replacing each internal vertex by a little black disc. Here is an illustration of the above procedure:
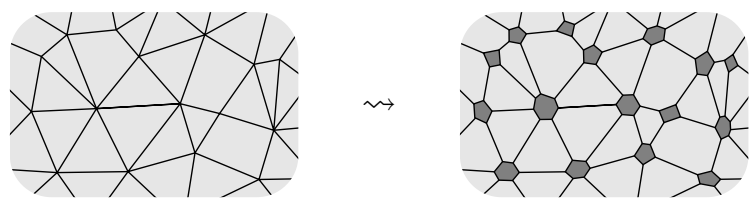

Given a soccer ball decomposition $X_{\text {white }}=\left\{\mathbb{D}_{1}, \ldots, \mathbb{D}_{n}\right\}, X_{\text {black }}=\left\{B_{1}, \ldots, B_{m}\right\}$ of our surface $\Sigma$, we can pick an order on those two sets, and apply the construction (2.2) to get

$$
\begin{gathered}
=\left(\left(\left(\cdots\left(H_{0}\left(\partial \mathbb{D}_{1}\right) \underset{\mathcal{A}\left(\partial \mathbb{D}_{2} \cap \mathbb{D}_{1}\right)}{\otimes} H_{0}\left(\partial \mathbb{D}_{2}\right)\right) \underset{\mathcal{A}\left(\partial \mathbb{D}_{3} \cap\left(\mathbb{D}_{1} \cup \mathbb{D}_{2}\right)\right)}{\otimes} \cdots\right) \underset{\mathcal{A}\left(\partial \mathbb{D}_{n} \cap \ldots\right)}{\otimes} H_{0}\left(\partial \mathbb{D}_{n}\right)\right)\right. \\
\left.\left.\underset{\mathcal{A}\left(\partial B_{1}\right)}{\otimes} H_{0}\left(\partial B_{1}\right)\right) \underset{\mathcal{A}\left(\partial B_{2}\right)}{\otimes} H_{0}\left(\partial B_{2}\right) \cdots\right) \underset{\mathcal{A}\left(\partial B_{m}\right)}{\otimes} H_{0}\left(\partial B_{m}\right) .
\end{gathered}
$$

The advantage of soccer ball decompositions is that, given choices of vacuum sectors $H_{0}\left(\partial \mathbb{D}_{i}\right)$ and $H_{0}\left(\partial B_{j}\right)$, the sector $V(\Sigma) \in \operatorname{Sect}_{\partial \Sigma}(\mathcal{A})$ is visibly invariant (up 
to canonical unitary isomorphism) under permutations of the sets $\left\{\mathbb{D}_{1}, \ldots, \mathbb{D}_{n}\right\}$ and $\left\{B_{1}, \ldots, B_{m}\right\}$ - this is seen as follows. The iterated fusion

$$
\left(\left(\ldots\left(H_{0}\left(\partial \mathbb{D}_{1}\right) \bigotimes_{\mathcal{A}\left(\partial \mathbb{D}_{2} \cap \mathbb{D}_{1}\right)} H_{0}\left(\partial \mathbb{D}_{2}\right)\right) \bigotimes_{\mathcal{A}\left(\partial \mathbb{D}_{3} \cap \ldots\right)} \cdots\right) \bigotimes_{\mathcal{A}\left(\partial \mathbb{D}_{n} \cap \ldots\right)} H_{0}\left(\partial \mathbb{D}_{n}\right)\right)
$$

can be identified with the graph fusion $\bigotimes_{\left\{\mathcal{A}\left(\mathbb{D}_{i} \cap \mathbb{D}_{j}\right)\right\}}\left\{H_{0}\left(\partial \mathbb{D}_{i}\right)\right\}$ of the Hilbert spaces $H_{0}\left(\partial \mathbb{D}_{i}\right)$ along the algebras $\mathcal{A}\left(\mathbb{D}_{i} \cap \mathbb{D}_{j}\right)$ (see Appendix A regarding graph fusion). Here, the manifolds $\mathbb{D}_{i} \cap \mathbb{D}_{j}$ are equipped with the orientation induced from $\partial \mathbb{D}_{j}$ for $j>i$, the graph that indexes the graph fusion is the adjacency graph $\Gamma$ of the white cells of $X$, and the orientations on $\Gamma$ are induced by the ordering of its set of vertices. Renumbering the cells $\mathbb{D}_{1}, \ldots, \mathbb{D}_{n}$ affects both the orientations of the $\mathbb{D}_{i} \cap \mathbb{D}_{j}$, and the orientations of the edges of $\Gamma$. It follows from (A.2) that those permutations leave (2.11) invariant. Altogether, $V(\Sigma)$ is canonically isomorphic to

$$
\left(\bigotimes_{\left\{\mathcal{A}\left(\mathbb{D}_{i} \cap \mathbb{D}_{j}\right)\right\}}\left\{H_{0}\left(\partial \mathbb{D}_{i}\right)\right\}\right) \bigotimes_{\mathcal{A}\left(\partial B_{1} \cup \ldots \cup \partial B_{m}\right)}\left(H_{0}\left(\partial B_{1}\right) \otimes \cdots \otimes H_{0}\left(\partial B_{m}\right)\right),
$$

and is therefore also invariant under the permutations of the cells $B_{1}, \ldots, B_{m}$.

By the above discussion, given a topological surface $\Sigma$ with smooth boundary, and a soccer ball decomposition $X=\left(X_{\text {white }}, X_{\text {black }}\right)$ of that surface, one can associate to it, canonically up to canonical-up-to-phase unitary isomorphism, a $\partial \Sigma$-sector $V(\Sigma)$. We denote the result

$$
V(\Sigma ; X)=V\left(\Sigma ; X_{\text {white }}, X_{\text {black }}\right) \in \operatorname{Sect}_{\partial \Sigma}(\mathcal{A})
$$

to emphasize the dependence on the soccer ball decomposition. The Hilbert space $V(\Sigma ; X)$ is only well defined up to canonical-up-to-phase unitary, because so were the vacuum sectors $H_{0}\left(\mathbb{D}_{i}\right)$ and $H_{0}\left(B_{j}\right)$ that entered its definition.

Our next goal is to show that $V(\Sigma ; X)$ does not depend on the soccer ball decomposition $X$.

Definition 2.14. Let $\Sigma$ be a surface with smooth boundary, and let $X$ and $Y$ be soccer ball decompositions. We write $X \stackrel{\mathbb{P}}{\prec} Y$ with $\mathbb{D} \in Y_{\text {white }}$ if $\mathbb{D}$ is a union of cells of $X$, the decompositions $X$ and $Y$ agree outside of $\mathbb{D}$, and the inclusion of the 1-skeleton of $Y$ into the 1-skeleton of $X$ is smooth. If there exists another soccer ball decomposition $Z$ and a cell $\mathbb{D} \in Z_{\text {white }}$ such that $X \stackrel{\mathbb{D}}{\prec} Z \stackrel{\mathbb{D}}{\succ} Y$, then we write $X \stackrel{\mathbb{D}}{\sim} Y$. For example, we have
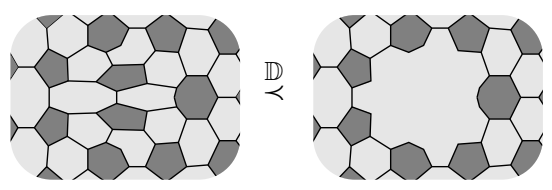

and
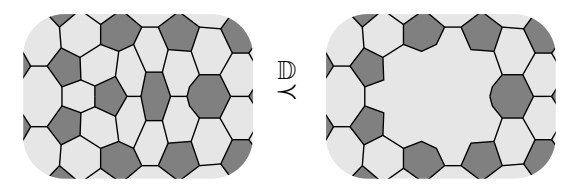

for the same disk $\mathbb{D}$, from which it follows that
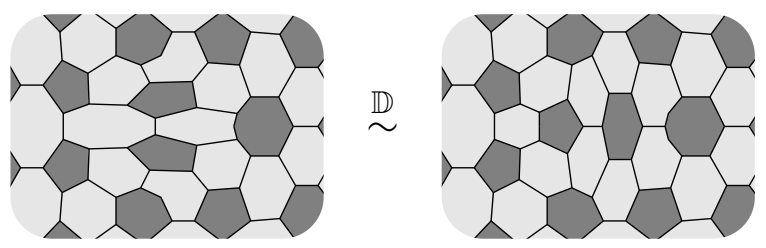

with

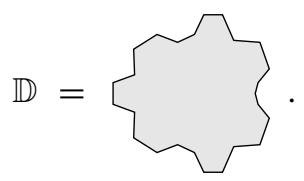


Given a set $S=\left\{\mathbb{D}_{0}, \ldots, \mathbb{D}_{n}\right\}$ of discs with disjoint interiors, we also write $X \stackrel{S}{\sim} Y$, or simply $X \sim Y$, if there exist soccer ball decompositions $Z_{1}, \ldots, Z_{n}$ such that

$$
X \stackrel{\mathbb{D}_{0}}{\sim} Z_{1} \stackrel{\mathbb{D}_{1}}{\sim} \ldots \stackrel{\mathbb{D}_{n-1}}{\sim} Z_{n} \stackrel{\mathbb{D}_{n}}{\sim} Y
$$

Finally, we write $X \stackrel{S}{\prec} Y$, or simply $X \prec Y$, if $S=\left\{\mathbb{D}_{0}, \ldots, \mathbb{D}_{n}\right\}$ is a collection of white cells of $Y$ and $X \stackrel{\mathbb{D}_{0}}{\prec} Z_{1} \stackrel{\mathbb{D}_{1}}{\prec} \ldots \stackrel{\mathbb{D}_{n-1}}{\prec} Z_{n} \stackrel{\mathbb{D}_{n}}{\prec} Y$ for some choice $Z_{1}, \ldots, Z_{n}$ of soccer ball decompositions. Note that $\prec$ is a transitive relation.

If $X \stackrel{\mathbb{D}}{\prec} Y$, we can construct a unitary isomorphism

$$
\phi_{X Y}: V(\Sigma ; X) \rightarrow V(\Sigma ; Y)
$$

well defined up to phase. Let us number the set $X_{\text {white }}=\left\{\mathbb{D}_{1}, \ldots, \mathbb{D}_{n}\right\}$ of white cells of $X$ in such a way that $\mathbb{D}_{i} \in Y_{\text {white }}$ for $i \leq p$ and $\mathbb{D}_{i} \subset \mathbb{D}$ for $i>p$. Similarly, we number the set $X_{\text {black }}=\left\{B_{1}, \ldots, B_{m}\right\}$ so as to have $B_{j} \in Y_{\text {black }}$ for $j \leq q$ and $B_{j} \subset \mathbb{D}$ for $j>q$. Letting $M:=\partial \mathbb{D} \cap\left(\mathbb{D}_{1} \cup \cdots \cup \mathbb{D}_{p}\right)$, the map $\phi_{X Y}$ is then defined as the composite

$$
\begin{gathered}
\phi_{X Y}: V(\Sigma ; X)=\left(\bigotimes_{\left\{\mathcal{A}\left(\mathbb{D}_{i} \cap \mathbb{D}_{j}\right)\right\}}\left\{H_{0}\left(\partial \mathbb{D}_{i}\right)\right\}_{i, j \leq n}\right) \otimes_{\mathcal{A}\left(\cup_{k \leq m} \partial B_{k}\right)}\left(\bigotimes_{k \leq m} H_{0}\left(\partial B_{k}\right)\right) \\
\stackrel{\cong}{\cong}\left(\left(\bigotimes_{\left\{\mathcal{A}\left(\mathbb{D}_{i} \cap \mathbb{D}_{j}\right)\right\}}\left\{H_{0}\left(\partial \mathbb{D}_{i}\right)\right\}_{i, j \leq p}\right) \underset{\mathcal{A}(M)}{\otimes}\left(\bigotimes_{\left\{\mathcal{A}\left(\mathbb{D}_{i} \cap \mathbb{D}_{j}\right)\right\}}\left\{H_{0}\left(\partial \mathbb{D}_{i}\right)\right\}_{p<i, j \leq n}\right)\right. \\
\left.\bigotimes_{\mathcal{A}\left(\cup_{q<k \leq m} \partial B_{k}\right)}\left(\bigotimes_{q<k \leq m} H_{0}\left(\partial B_{k}\right)\right)\right) \bigotimes_{\mathcal{A}\left(\cup_{k \leq q} \partial B_{k}\right)}\left(\bigotimes_{k \leq q} H_{0}\left(\partial B_{k}\right)\right) \\
\stackrel{\cong}{\cong}\left(\left(\bigotimes_{\left\{\mathcal{A}\left(\mathbb{D}_{i} \cap \mathbb{D}_{j}\right)\right\}}\left\{H_{0}\left(\partial \mathbb{D}_{i}\right)\right\}_{i, j \leq p}\right) \underset{\mathcal{A}(M)}{\bigotimes} H_{0}(\partial \mathbb{D})\right) \bigotimes_{\mathcal{A}\left(\cup_{k \leq q} \partial B_{k}\right)}\left(\bigotimes_{k \leq q} H_{0}\left(\partial B_{k}\right)\right)
\end{gathered}
$$

where the first isomorphism is an instance of (A.3), and the second one follows from Corollary 2.9. If $X, Y, Z$ are soccer ball decompositions such that $X \stackrel{\mathbb{P}}{\prec} Y \stackrel{\mathbb{D}^{\prime}}{\prec} Z$ and $X \stackrel{\mathbb{D}^{\prime}}{\prec} Z$, then it follows from Corollary 2.9 that

$$
\phi_{Y Z} \circ \phi_{X Y}=\phi_{X Z}
$$

up to phase. If two soccer ball decompositions are related by $X \prec Y$ then, by composing suitable instances of (2.16), we obtain an induced unitary isomorphism $\phi_{X Y}$ : $V(\Sigma ; X) \rightarrow V(\Sigma ; Y)$, well defined up to phase. As before, whenever $X \prec Y \prec Z$, those isomorphisms satisfy $\phi_{Y Z} \circ \phi_{X Y}=\phi_{X Z}$ up to phase.

If $X$ and $Y$ are soccer ball decompositions related by $X \stackrel{\mathbb{D}}{\sim} Y$, then we also define

$$
\Phi_{\mathbb{D}}: V(\Sigma ; X) \rightarrow V(\Sigma ; Y)
$$

to be the composite $\phi_{Y Z}^{-1} \circ \phi_{X Z}$, where $Z$ is such that $X \stackrel{\mathbb{D}}{\prec} Z \stackrel{\mathbb{D}}{\succ} Y$. The map $\Phi_{\mathbb{D}}$ is well defined up to phase but, a priori, it depends on the choice of disk $\mathbb{D}$. 
Main Theorem 2.18. Given a conformal net $\mathcal{A}$ with finite index, there exists a construction

$$
\Sigma \mapsto V(\Sigma) \in \operatorname{Sect}_{\partial \Sigma}(\mathcal{A}),
$$

that assigns, to every compact oriented topological surface $\Sigma$ with smooth boundary, a sector $V(\Sigma)=V(\Sigma ; \mathcal{A})$, well defined up to canonical-up-to-phase unitary isomorphism. Moreover, if the surface $\Sigma$ is a disc, there exists a unitary isomorphism, canonical up to phase, between $V(\Sigma)$ and the vacuum sector $H_{0}(\partial \Sigma)$.

Given surfaces $\Sigma_{1}, \Sigma_{2}$ as above, and a homeomorphism $f: \Sigma_{1} \rightarrow \Sigma_{2}$ that is either orientation preserving or orientation reversing (which is automatic if $\Sigma_{1}$ is connected), and smooth on the boundary, there is an induced map

$$
V(f): V\left(\Sigma_{1}\right) \rightarrow V\left(\Sigma_{2}\right)
$$

well defined up to phase. The map $V(f)=V(f, \mathcal{A})$ is complex linear if $f$ is orientation preserving and complex antilinear if $f$ is orientation reversing. The above construction satisfies $V(f \circ g)=V(f) \circ V(g)$ up to phase for composable $f$ and $g$. Moreover, if $f_{1}, f_{2}: \Sigma_{1} \rightarrow \Sigma_{2}$ are isotopic relative to $\partial \Sigma_{1}$, then $V\left(f_{1}\right)=V\left(f_{2}\right)$, up to phase.

Given an interval $I \subset \partial \Sigma_{1}$ and an element $a \in \mathcal{A}(I)$, the map $V(f)$ satisfies $V(f) a=\mathcal{A}\left(\left.f\right|_{I}\right)(a) V(f)$ if $f$ is orientation preserving and $V(f) a=\mathcal{A}\left(\left.f\right|_{I}\right)\left(a^{*}\right) V(f)$ if $f$ is orientation reversing.

Finally, the map $f \mapsto V(f)$ is continuous for the natural topology on the set of homeomorphisms $f: \Sigma_{1} \rightarrow \Sigma_{2}$ that are smooth on the boundary modulo isotopy rel boundary, ${ }^{4}$ and the strong topology on $\mathrm{PU}_{ \pm}(V(\Sigma))$.

Part of the content of the above theorem can be understood as follows. Let 2MAN be the following groupoid:

2MAN $:= \begin{cases}\text { Objects: } \begin{array}{l}\text { Compact oriented topological surfaces equipped with a smooth structure } \\ \text { on their boundary. }\end{array} \\ \text { Isotopy classes of homeomorphisms that are either orientation preserving } \\ \text { Morphisms: } \begin{array}{l}\text { or orientation reversing, and that are smooth on the boundary, where the } \\ \text { isotopies are taken relative to the boundary. }\end{array}\end{cases}$

and let pHILB be the groupoid whose objects are complex Hilbert space, and whose morphisms are the projective unitary and the projective antiunitary maps. Then there is a symmetric monoidal continuous functor ${ }^{5}$

$$
V: 2 \mathrm{MAN} \rightarrow \text { pHILB }
$$

It is interesting to note that in the groupoid 2MAN, the automorphism group of the unit disk is $\operatorname{Diff}\left(S^{1}\right)$, and so $V$ recovers the projective action of $\operatorname{Diff}\left(S^{1}\right)$ on the vacuum sector of $\mathcal{A}$. At another extreme, the automorphism group of a closed surface $\Sigma$ is its mapping class group. We will see in Sect. 2.F that in the case of a closed surface the Hilbert space $V(\Sigma)$ is finite dimensional. We conjecture that it agrees with the spaces of conformal blocks, as defined in conformal field theory. By applying the functor $V$, one therefore recovers the projective action of the mapping class group on the spaces of conformal

\footnotetext{
4 The topology here is the quotient topology on Pullback (Homeo( $\left.\Sigma_{1}, \Sigma_{2}\right) \rightarrow \operatorname{Homeo}\left(\partial \Sigma_{1}, \partial \Sigma_{2}\right) \leftarrow$ $\left.\operatorname{Diff}\left(\partial \Sigma_{1}, \partial \Sigma_{2}\right)\right) / \sim$ where Homeo is equipped with the $\mathcal{C}^{0}$ topology, and Diff is equipped with the $\mathcal{C}^{\infty}$ topology.

5 The comments in Footnote 3 also apply to the functor $V$.
} 
blocks. For a general surface, the automorphism group $G(\Sigma):=\operatorname{Aut}_{2 \mathrm{MAN}}(\Sigma)$ fits into a short exact sequence

$$
1 \rightarrow \Gamma(\Sigma) \rightarrow G(\Sigma) \rightarrow D \rightarrow 1
$$

of topological groups, where $D$ is the finite index subgroup of diffeomorphisms of $\partial \Sigma$ that admit an extension to all of $\Sigma$, and $\Gamma(\Sigma)$ is the mapping class group of $\Sigma$ relative to its boundary, equipped with the discrete topology. This group $G(\Sigma)$ was already considered in [34, Thm. 2.11]; we call it the extended mapping class group of $\Sigma$. As a corollary of the above theorem, the extended mapping class group $G(\Sigma)$ admits a continuous projective (anti)unitary action on the Hilbert space $V(\Sigma)$.

Proof of Theorem 2.18. We apply the strategy outlined in Sect. 2.A, and first construct the definition complex $\mathbb{X}_{\Sigma}$ associated to $V(\Sigma)$. The vertices of $\mathbb{X}_{\Sigma}$ are the soccer ball decompositions of $\Sigma$. The edges between two vertices $X$ and $Y$ are the discs $\mathbb{D} \subset \Sigma$ such that $X \stackrel{\mathbb{D}}{\sim} Y$. Finally, there is a 2-cell of $\mathbb{X}_{\Sigma}$ attached to the loop

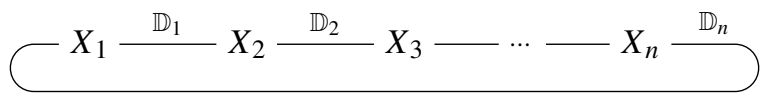

if there exists a soccer ball decomposition $Z$ such that $X_{i} \prec Z$ for all $i$, and such that every $\mathbb{D}_{i}$ is contained in some white cell of $Z$.

We have seen in (2.13) how to construct a sector $V(\Sigma ; X) \in \operatorname{Sect}_{\partial \Sigma}(\mathcal{A})$ for every vertex $X$ of $\mathbb{X}_{\Sigma}$, and we have seen in (2.17) how to construct a unitary isomorphism $\Phi_{\mathbb{D}}: V(\Sigma ; X) \rightarrow V(\Sigma ; Y)$ for every edge $\mathbb{D}$ of $\mathbb{X}_{\Sigma}$. Given a 2-cell (2.23), let $Y_{i}$ and $Z$ be soccer ball decompositions such that $X_{i} \stackrel{\mathbb{D}_{i}}{\prec} Y_{i} \stackrel{\mathbb{D}_{i}}{\succ} X_{i+1}$ and $Y_{i} \prec Z$. The above relations induce diagrams
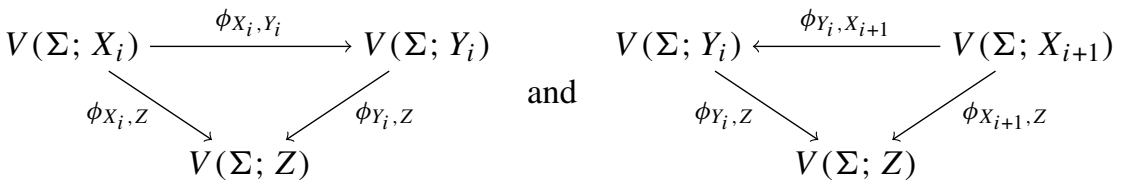

that commute up to phase. When assembled together, they show that the composite

$$
V\left(\Sigma ; X_{1}\right) \stackrel{\Phi_{\mathbb{D}_{1}}}{\longrightarrow} V\left(\Sigma ; X_{2}\right) \stackrel{\Phi_{\mathbb{D}_{2}}}{\longrightarrow} \cdots \stackrel{\Phi_{\mathbb{D}_{n-1}}}{\longrightarrow} V\left(\Sigma ; X_{n}\right) \stackrel{\Phi_{\mathbb{D}_{n}}}{\longrightarrow} V\left(\Sigma ; X_{1}\right)
$$

is a scalar multiple of the identity, as desired.

To finish showing that $V(\Sigma)$ is well defined up to canonical-up-to-phase unitary isomorphism, we need to prove that $\mathbb{X}_{\Sigma}$ is simply connected: this is the content of Lemma 2.25. Finally, if $\Sigma$ is a disc, there is a unitary isomorphism canonical up to phase $V(\Sigma) \cong H_{0}(\partial \Sigma)$ by Corollary 2.9 .

Given a homeomorphism $f: \Sigma_{1} \rightarrow \Sigma_{2}$ that is smooth on the boundary, a soccer ball decomposition $X$ of $\Sigma_{1}$ induces a corresponding soccer ball decomposition $f_{*} X$ of $\Sigma_{2}$. If $\mathbb{D}$ is a cell of $X$, then the identity map from $H_{0}(\partial \mathbb{D}) \in \operatorname{Sect}_{\partial \mathbb{D}}(\mathcal{A})$ to $\left(f^{-1}\right)^{*}\left(H_{0}(\partial \mathbb{D})\right) \in$ $\operatorname{Sect}_{f(\partial \mathbb{D})}(\mathcal{A})$ is complex linear if $f$ is orientation preserving, and complex antilinear otherwise. The sector $\left(f^{-1}\right)^{*}\left(H_{0}(\partial \mathbb{D})\right)$ is isomorphic to $H_{0}(f(\partial \mathbb{D}))$, and so we get a 
map $H_{0}(f): H_{0}(\partial \mathbb{D}) \rightarrow H_{0}(f(\partial \mathbb{D}))$, canonical up to phase. By construction, these maps satisfy

$$
\begin{cases}H_{0}(f) a=\mathcal{A}\left(\left.f\right|_{I}\right)(a) H_{0}(f) & \text { if } f \text { is orientation preserving } \\ H_{0}(f) a=\mathcal{A}\left(\left.f\right|_{I}\right)\left(a^{*}\right) H_{0}(f) & \text { if } f \text { is orientation reversing }\end{cases}
$$

for every interval $I \subset \partial \mathbb{D}$ and $a \in \mathcal{A}(I)$.

Applying the maps $H_{0}(f)$ to every occurrence of a vacuum sector in (2.12), we get an induced map $V(f ; X): V\left(\Sigma_{1} ; X\right) \rightarrow V\left(\Sigma_{2} ; f_{*} X\right)$ which satisfies the same equivariance properties (2.24) as the maps $H_{0}(f)$ used in its construction, but now for $I \subset \partial \Sigma$. For every edge $X \stackrel{\mathbb{D}}{\sim} Y$ of the definition complex $\mathbb{X}_{\Sigma_{1}}$, there is a corresponding edge $f_{*} X \stackrel{f(\mathbb{D})}{\sim} f_{*} Y$ of $\mathbb{X}_{\Sigma_{2}}$. The diagrams

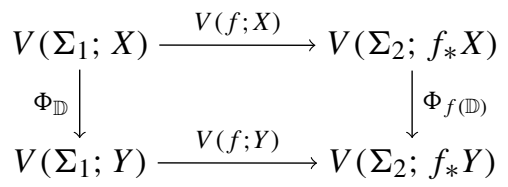

are commutative, and so the maps $V(f ; X)$ induce a map $V(f): V\left(\Sigma_{1}\right) \rightarrow V\left(\Sigma_{2}\right)$, well defined up to phase. The relation $V(f \circ g)=V(f) \circ V(g)$ is immediate from the definition.

Next, we need to show that if $f$ is isotopic to the identity, then $V(f)=\operatorname{Id}_{V(\Sigma)}$. Write $f$ as a composition of homeomorphisms $f_{1} \circ \cdots \circ f_{n}$ such that for each $i$ there is a disk $D_{i} \subset \Sigma$ that contains the support of $f_{i}$. Since $V(f)=V\left(f_{1}\right) \circ \cdots \circ V\left(f_{n}\right)$, it is enough to show that each $V\left(f_{i}\right)$ is the identity. Pick a soccer ball decomposition $X_{i}$ whose 1-skeleton does not intersect $D_{i}$. The definition of $V\left(\Sigma ; X_{i}\right)$ only uses the 1-skeleton of $X_{i}$, and so, clearly, $V\left(f_{i} ; X_{i}\right): V\left(\Sigma ; X_{i}\right) \rightarrow V\left(\Sigma ; X_{i}\right)$ is the identity. If follows that $V\left(f_{i}\right): V(\Sigma) \rightarrow V(\Sigma)$ is the identity, and therefore so is $V(f)$.

Finally, we show that the assignment $f \mapsto V(f)$ is continuous, which is equivalent to showing that for every surface $\Sigma$, the homomorphism

$$
G(\Sigma) \rightarrow \mathrm{PU}_{ \pm}(V(\Sigma))
$$

is continuous, where $G(\Sigma)$ is the group defined in (2.22). Let $S_{1}, \ldots, S_{n}$ be the boundary components of $\Sigma$, and let $\widetilde{\operatorname{Diff}}_{+}\left(S_{i}\right)$ denote the universal cover of Diff $+\left(S_{i}\right)$. Then $\prod \widetilde{\mathrm{Diff}}_{+}\left(S_{i}\right)$ admits a natural homomorphism to $G(\Sigma)$, which is a homeomorphism on a neighborhood of the identity. It is therefore enough to check the continuity of the map $\prod \widetilde{\mathrm{Diff}}_{+}\left(S_{i}\right) \rightarrow \mathrm{PU}_{ \pm}(V(\Sigma))$. Equivalently, we have to show that each map $\widetilde{\operatorname{Diff}}_{+}\left(S_{i}\right) \rightarrow \mathrm{PU}_{ \pm}(V(\Sigma))$ is continuous. For every choice of intervals $K \subset I \subset S_{i}$, let us write $\operatorname{Diff}_{0, K}(I)$ for the group of diffeomorphisms of $I$ that fix the complement of $K$ pointwise. The topology on $\widetilde{\operatorname{Diff}}_{+}\left(S_{i}\right)$ is the finest one for which all the inclusions $\operatorname{Diff}_{0, K}(I) \hookrightarrow \operatorname{Diff}\left(S_{i}\right)$ are continuous. It is therefore sufficient to show that the homomorphisms Diff ${ }_{0, K}(I) \rightarrow \mathrm{PU}(V(\Sigma))$ are continuous. To finish the argument, we note that the above homomorphisms can be factored as

$$
\operatorname{Diff}_{0, K}(I) \rightarrow \operatorname{PU}(\mathcal{A}(I)) \rightarrow \mathrm{PU}(V(\Sigma)),
$$

where the first map is continuous by [8, Lemma 2.11], and the second one is continuous because $V(\Sigma)$ is a $\partial \Sigma$-sector. 
Lemma 2.25. The cell complex $\mathbb{X}_{\Sigma}$ is connected and simply connected.

Proof. We will show that given a finite graph $\Gamma$ in the 1 -skeleton of $\mathbb{X}_{\Sigma}$, the inclusion map $\iota: \Gamma \hookrightarrow \mathbb{X}_{\Sigma}$ extends to the cone on $\Gamma$ :

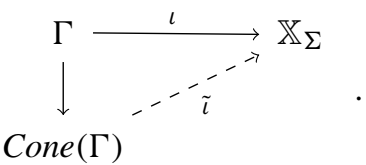

The case when $\Gamma$ consists of just two points shows that $\mathbb{X}_{\Sigma}$ is connected, and the case when $\Gamma$ is a loop shows that $\mathbb{X}_{\Sigma}$ is simply connected.

In order to construct the extension $\tilde{\iota}$, we need to: (1) pick a vertex $Y$ of $\mathbb{X}_{\Sigma}$, (2) provide a path $\gamma_{X}$ from $X$ to $Y$ for every vertex $X \in \Gamma$, and (3) for every edge $X_{1} \stackrel{\mathbb{D}}{\sim} X_{2}$ of $\Gamma$, construct a map $\delta_{\mathbb{D}}: D^{2} \rightarrow \mathbb{X}_{\Sigma}$ that bounds the triangle

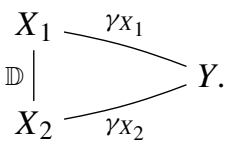

(1) We first construct a soccer ball decomposition $Y$ as follows. Start by picking a cell decomposition $Y_{1}$ (no soccer ball structure) that is transverse to all the decompositions $X_{i} \in \Gamma{ }^{6}$ It is taken fine enough so that it contains vertices in each face of each $X_{i}$, and so that it has at least one edge intersecting every edge of every $X_{i}$. Add extra edges and vertices if necessary so that the intersection of the 1-skeleton of $Y_{1}$ with every 2-cell of every $X_{i}$ remains connected. ${ }^{7}$ Now double every edge and call the result $Y_{2}$. The cell decomposition $Y_{2}$ has the same vertices as $Y_{1}$, but it has a new bigon face in the place of every edge of $Y_{1}$. Finally, replace the vertices by little black disks, and equip the result with a smooth structure, so as to get a soccer ball decomposition. The black disks are chosen small enough, so as to not intersect the edges of the $X_{i}$.

We give an illustration of the above process. The soccer ball decomposition denoted $X$ below represents one of the vertices of $\Gamma$, and the cell decomposition $Y_{1}$ is the beginning point of our construction:
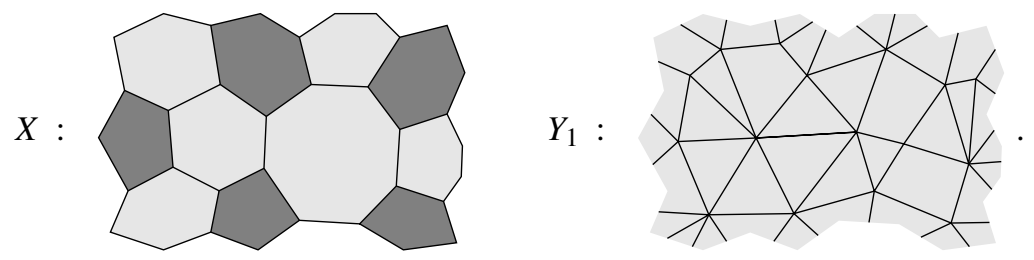

\footnotetext{
6 See http://mathoverflow.net/questions/176227/topological-transversality for a proof that this always exists.

7 Caveat: Even though we believe that this is possible, we don't actually know how to construct $Y_{1}$ with this last property. What we can achieve instead by adding edges to $Y_{1}$ is that for each 2-cell of each $X_{i}$, the intersection of the 2-cell with the 1-skeleton of $Y_{1}$ has one 'big' connected component, that touches every edge of the cell. In order not to overcomplicate our argument, we will proceed assuming the intersection of the 1-skeleton of $Y_{1}$ with every 2-cell of every $X_{i}$ is actually connected. At the end of the proof, in footnote 8 , we address the issue by indicating the necessary modification to the argument in case those intersections are not connected.
} 
It is convenient to draw $Y_{1}$ on top of $X$ in order to check that they satisfy the desired properties:

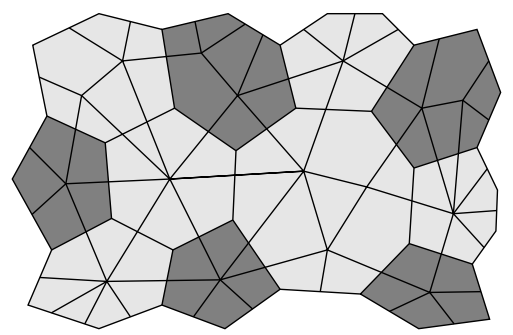

The cell decomposition $Y_{1}$ is transverse to $X$, it intersects every edge of $X$, and its 1 skeleton remains connected when intersected with every 2-cell of $X$. It is therefore a valid beginning point for our construction (as far as $X$ is concerned). The next steps are then as follows:

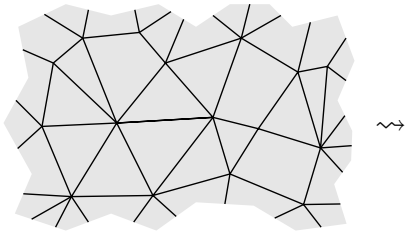

$Y_{1}$

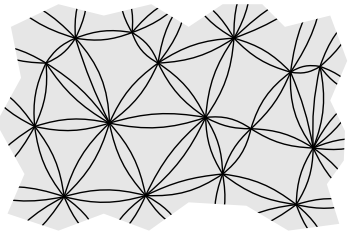

$Y_{2}$

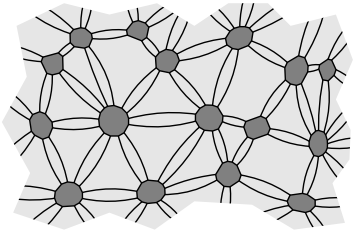

Y

By superimposing the soccer ball decompositions $X$ and $Y$ we obtain:

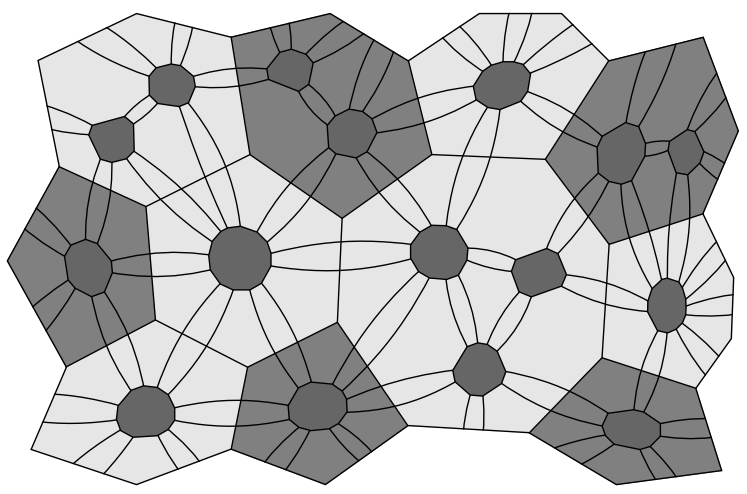

(2) For every $X \in \Gamma$, we now describe two related soccer ball decompositions $X^{\prime}$ and $X^{\prime \prime}$ that satisfy

$$
X \sim X^{\prime} \sim X^{\prime \prime} \sim Y
$$

Each one of the three 's above corresponds, as in (2.15), to a path in $\mathbb{X}_{\Sigma}$ and we call their concatenation $\gamma_{X}$.

Let $A$ be the set of edges of $X$ that separate two white cells, and let $B$ be the set of edges of $Y$ that intersect edges in $A$. The first soccer ball decomposition $X^{\prime}$ is defined as follows. Its 1-skeleton is the union of the 1-skeleton of $X$ and of the edges $e \cap \mathbb{D}$, where 
$\mathbb{D}$ is a white cell of $X$, and $e$ is an edge of $Y, e \notin B$. The soccer ball structure on $X^{\prime}$ is inherited from that of $Y$, and the smooth structure around the new trivalent vertices (at the intersection of the 1-skeletons of $X$ and of $Y$ ) is chosen arbitrarily. The second soccer ball decomposition $X^{\prime \prime}$ is then obtained from $X^{\prime}$ by removing all the edges in $A$, and adding all those in $B$.

In the example when $X$ and $Y$ are as in (2.28), then the intermediate soccer ball decompositions $X^{\prime}$ and $X^{\prime \prime}$ appear as follows:

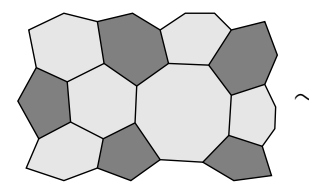

$X$

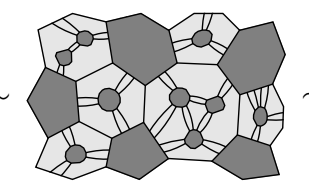

$X^{\prime}$

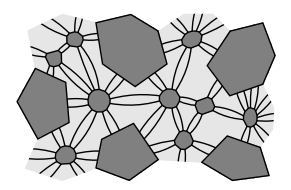

$X^{\prime \prime}$

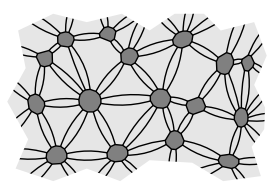

Y

(3) Finally, given an edge $X_{1} \stackrel{\mathbb{D}}{\sim} X_{2}$ of $\Gamma$, we construct the map $\delta_{\mathbb{D}}: D^{2} \rightarrow \mathbb{X}_{\Sigma}$ that bounds the triangle (2.27). That triangle can be decomposed as follows

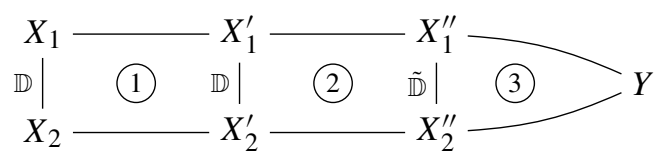

and we claim that each one of the above cycles (1), (2), (3) bounds a 2-cell of $\mathbb{X}_{\Sigma}$. The union of those three 2-cells provides the desired filler $\delta_{\mathbb{D}}$.

By the definition of $X_{1} \stackrel{\mathbb{D}}{\sim} X_{2}$, there is a soccer ball decomposition $Z$ such that $X_{1} \stackrel{\mathbb{D}}{\prec} Z \stackrel{\mathbb{D}}{\succ} X_{2}$. The first loop (1) bounds a 2-cell because

$$
X_{1} \prec Z, \quad X_{2} \prec Z, \quad X_{1}^{\prime} \prec Z, \quad \text { and } \quad X_{2}^{\prime} \prec Z .
$$

Let $\tilde{\mathbb{D}} \subset \Sigma$ be the union of all the cells of $X_{1}^{\prime \prime}$ (equivalently $X_{2}^{\prime \prime}$ ) whose interior intersects $\mathbb{D}$. It follows from the construction of $Y$ that $\tilde{\mathbb{D}}$ is a disc. Let $Z^{\prime}$ be the soccer ball decomposition obtained from $X_{1}^{\prime}$ (equivalently $X_{2}^{\prime}$ ) by removing all the edges $e \in A$, and all the edges and vertices that are in the interior of $\mathbb{D}$. Note that $\tilde{\mathbb{D}}$ is one of the cells of $Z^{\prime}$. We then have

$$
X_{1}^{\prime} \prec Z^{\prime}, \quad X_{2}^{\prime} \prec Z^{\prime}, \quad X_{1}^{\prime \prime} \prec Z^{\prime}, \quad \text { and } \quad X_{2}^{\prime \prime} \prec Z^{\prime},
$$

and so the second loop (2) also bounds a 2-cell. To finish the argument, we need to construct a soccer ball decomposition $Z^{\prime \prime}$ such that

$$
X_{1}^{\prime \prime} \prec Z^{\prime \prime}, \quad X_{2}^{\prime \prime} \prec Z^{\prime \prime}, \quad \text { and } \quad Y \prec Z^{\prime \prime} .
$$

This will then show that the third loop (3) bounds a 2-cell.

Let $B_{1}, \ldots, B_{n}$ be the set of black cells of $X_{1}^{\prime \prime}$ (equivalently $X_{2}^{\prime \prime}$ ) that are not contained in $\mathbb{D}$. For each one of them, let $\tilde{B}_{i}$ be the union of all the cells of $Y$ that intersect $B_{i}$. It follows from the construction of $Y$ that $\tilde{B}_{i}$ is a disc. Let $U \subset \mathbb{D}$ be the union of all the cells of $Y$ that intersect some black cell $B \subset \mathbb{D}$ of $X_{1}$ or $X_{2}$ and let $U_{1}, \ldots, U_{k}$ be the connected components of $U$. Finally, let $D_{j}$ be the smallest disk that is contained in $\mathbb{D}$ and that contains $U_{j}$. It follows from the construction of $Y$ that the set $S:=$ 
$\left\{\tilde{B}_{1}, \ldots, \tilde{B}_{n}, D_{1}, \ldots, D_{k}\right\}$ consists of pairwise disjoint discs. Moreover, it defines a unique soccer ball decomposition $Z^{\prime \prime}$ such that $X_{1}^{\prime \prime} \stackrel{S}{\prec} Z^{\prime \prime}, X_{2}^{\prime \prime} \stackrel{S}{\prec} Z^{\prime \prime}$, and $Y \stackrel{S}{\prec} Z^{\prime \prime}$. This finishes the proof that $\mathbb{X}_{\Sigma}$ is simply connected. ${ }^{8}$

2.E. Factorization along circles and along intervals. From now on, all surfaces will be assumed to be compact oriented topological surfaces with smooth boundary.

Let $\Sigma_{1}$ and $\Sigma_{2}$ be two surfaces, pick an orientation-reversing diffeomorphism $\varphi$ : $M_{1} \rightarrow M$ between submanifolds $M_{1} \subset \partial \Sigma_{1}$ and $M \subset \partial \Sigma_{2}$, and equip the trivalent graph $\Gamma:=\partial \Sigma_{1} \cup_{M} \partial \Sigma_{2}$ with a smooth structure (Definition 1.4), compatibly with the existing smooth structures on $\partial \Sigma_{1}$ and $\partial \Sigma_{2}$. The smooth structure on $\Gamma$ restricts to a smooth structure on the boundary of $\Sigma_{1} \cup_{M} \Sigma_{2}$, and so we can define $V\left(\Sigma_{1} \cup_{M} \Sigma_{2}\right)$ by Theorem 2.18.

Main Theorem 2.29. Let $\Sigma_{1}, \Sigma_{2}, M_{1}, M, \varphi$ be as above. Then there is a unitary isomorphism

$$
g: V\left(\Sigma_{1} \cup_{M} \Sigma_{2}\right) \rightarrow V\left(\Sigma_{1}\right) \bigotimes_{\mathcal{A}(M)} V\left(\Sigma_{2}\right),
$$

well defined up to phase.

Before embarking on the proof of the theorem, we will need the following variation of the notion of soccer ball decomposition:

Definition 2.31. Let $\Sigma$ be surface, and let $S \subset \partial \Sigma$ be a submanifold of its boundary diffeomorphic to a disjoint union of circles. A regular trivalent smooth cell decomposition $X$ of $\Sigma$ (see Sect. 2.B), equipped with a partition of the set of 2-cells into subsets $X_{\text {white }}$ and $X_{\text {black }}$ is called an $S$-open soccer ball decomposition of $\Sigma$ if every interior vertex is adjacent to two white cells and one back cell, every boundary vertex not in $S$ is adjacent to two white cells, and every boundary vertex in $S$ is adjacent to a white and a black cell.

We shall sometimes abbreviate the above terminology by "open soccer ball decomposition" when the manifold $S$ is obvious from the context.

An example of an open soccer ball decomposition:

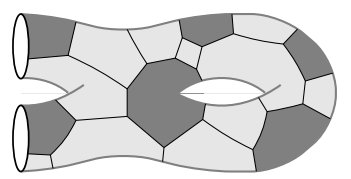

Note that open soccer ball decompositions are not soccer ball decompositions, unless $S=\emptyset$. Indeed, in a soccer ball decomposition, the cells along the boundary are all white, whereas is an open soccer ball decomposition, the cells along the submanifold $S$ alternate between black and white.

\footnotetext{
8 Caveat resolution: If one follows the definition of $X^{\prime}$ presented above, using a cell decomposition $Y_{1}$ whose 1-skeleton has a disconnected intersection with some 2-cell of some $X_{i}$, then one may encounter the following problem. Let $\mathbb{D}$ be a white cell of $X$, and let $e$ be an edge that separates it from some other white cell of $X$. If there exists a small component of the intersection of the 1-skeleton of $Y_{1}$ with $\mathbb{D}$ that only touches $e$, then the 1-skeleton of $X^{\prime}$ will be disconnected and $X^{\prime}$ won't be a soccer ball decomposition. However, we may simply redefine $X^{\prime}$ by removing those small components; this yields a new soccer ball decomposition which one uses in place of $X^{\prime}$. The rest of the argument is unaffected by this modification.
} 
Remark 2.32. Let $\Sigma$ be a surface, and let $S \subset \partial \Sigma$ be a disjoint union of circles. Let $\Delta=D_{1} \cup \cdots \cup D_{k}$ be a union of discs, and let $\Sigma^{+}:=\Sigma \cup_{\varphi} \Delta$ for some diffeomorphism $\varphi: S \rightarrow \partial \Delta$. Then a regular trivalent smooth cell decomposition $X$ of $\Sigma$, with a partition of the set of 2-cells into $X_{\text {white }}$ and $X_{\text {black }}$ is an $S$-open soccer ball decomposition if and only if ( $X_{\text {white }} \cup\left\{D_{1}, \ldots, D_{k}\right\}, X_{\text {black }}$ ) is a soccer ball decomposition of $\Sigma^{+}$.

Let $\Sigma$ and $\Sigma^{\prime}$ be surfaces, let $S$ be a closed 1-manifold equipped with orientation preserving embeddings $\bar{S} \rightarrow \partial \Sigma$ and $S \rightarrow \partial \Sigma^{\prime}$, where $\bar{S}$ denotes $S$ with the opposite orientation, and let $\Sigma^{+}=\Sigma \cup_{S} \Sigma^{\prime}$ be the result of gluing $\Sigma$ and $\Sigma^{\prime}$ along $S$. Given a soccer ball decomposition $X$ of $\Sigma$ and an $S$-open soccer ball decomposition $Y$ of $\Sigma^{\prime}$, we say that $X$ and $Y$ are compatible if $X \cup Y$ is a soccer ball decomposition of $\Sigma^{+}$. If we write the boundary of $\Sigma^{\prime}$ as $\partial \Sigma^{\prime}=S \sqcup T$, and we denote the elements of our open soccer ball decomposition by $Y_{\text {white }}=\left\{\mathbb{D}_{1}, \ldots, \mathbb{D}_{n}\right\}$ and $Y_{\text {black }}=\left\{B_{1}, \ldots, B_{m}\right\}$, then we may consider the functor

$$
\begin{aligned}
\operatorname{Sect}_{\bar{S}}(\mathcal{A}) & \rightarrow \operatorname{Sect}_{T}(\mathcal{A}) \\
H & \mapsto H \triangleright Y
\end{aligned}
$$

given by

$$
\begin{aligned}
H \triangleright Y:=\left(H \bigotimes_{\mathcal{A}\left(S \cap\left(\mathbb{D}_{1} \cup \ldots \cup \mathbb{D}_{n}\right)\right)}\left(\bigotimes_{\left\{\mathcal{A}\left(\mathbb{D}_{i} \cap \mathbb{D}_{j}\right)\right\}}\left\{H_{0}\left(\partial \mathbb{D}_{i}\right)\right\}\right)\right) \\
\bigotimes_{\mathcal{A}\left(\partial B_{1} \cup \ldots \cup \partial B_{m}\right)}\left(H_{0}\left(\partial B_{1}\right) \otimes \cdots \otimes H_{0}\left(\partial B_{m}\right)\right) .
\end{aligned}
$$

Note that if $X$ is a soccer ball decomposition that is compatible with $Y$, then we have a canonical unitary isomorphism

$$
V\left(\Sigma^{+} ; X \cup Y\right) \cong V(\Sigma ; X) \triangleright Y .
$$

Similarly, for $\Sigma^{+}=\Sigma \cup_{S} \Sigma^{\prime}$ as above, if $X$ is an $S$-open soccer ball decomposition of $\Sigma$ and $Y$ a compatible soccer ball decomposition of $\Sigma^{\prime}$, then

$$
V\left(\Sigma^{+} ; X \cup Y\right) \cong X \triangleleft V\left(\Sigma^{\prime} ; Y\right)
$$

for an analogously defined functor $X \triangleleft-$.

Lemma 2.34. Let $\Sigma^{+}=\Sigma \cup_{S} \Sigma^{\prime}$ be as above. Then for any $S$-open soccer ball decomposition $Y$ of $\Sigma^{\prime}$, the maps (2.33) induce unitary isomorphisms

$$
\lambda_{Y}: V\left(\Sigma^{+}\right) \rightarrow V(\Sigma) \triangleright Y,
$$

canonical up to phase.

Proof. Recall the definition complex $\mathbb{X}_{\Sigma}$ from the proof of Theorem 2.18, and let $\tilde{\mathbb{X}}_{\Sigma} \subset$ $\mathbb{X}_{\Sigma}$ be the subcomplex whose vertices are the soccer ball decompositions of $\Sigma$ that are compatible with $Y$. For such a soccer ball decomposition $X \in \tilde{\mathbb{X}}_{\Sigma}$, we may consider the composite

$$
\lambda_{X, Y}: V\left(\Sigma^{+}\right) \rightarrow V\left(\Sigma^{+} ; X \cup Y\right) \rightarrow V(\Sigma ; X) \triangleright Y \rightarrow V(\Sigma) \triangleright Y,
$$

where the middle map is the isomorphism (2.33), and the two outer ones are constructed in the proof of Theorem 2.18. 
We now show that $\lambda_{X, Y}$ is independent of $X$, up to phase. Recall the maps $\Phi_{\mathbb{D}}$ from (2.17). If $X_{1} \in \tilde{\mathbb{X}}_{\Sigma}$ is another compatible soccer ball decomposition related by $X_{1} \stackrel{\mathbb{D}}{\sim} X$, then it is clear by construction that the diagram

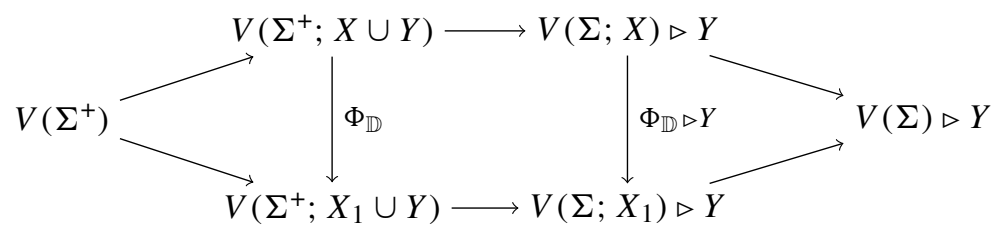

commutes up to phase, and so $\lambda_{X, Y}=\lambda_{X_{1}, Y}$ up to phase. The result follows because $\tilde{\mathbb{X}}_{\Sigma}$ is connected.

The isomorphisms (2.35) satisfy the following version of associativity. Given surfaces $\Sigma, \Sigma^{\prime}, \Sigma^{\prime \prime}$ and closed 1-manifolds $S$ and $T$ along with orientation preserving embeddings $\bar{S} \rightarrow \partial \Sigma, S \sqcup \bar{T} \rightarrow \Sigma^{\prime}$, and $T \rightarrow \partial \Sigma^{\prime \prime}$, then for every open soccer ball decompositions $X$ of $\Sigma$ and $Y$ of $\Sigma^{\prime \prime}$, the diagram

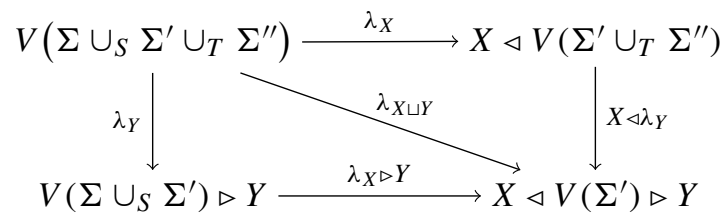

commutes up to phase.

Proof of Theorem 2.29. We first deal with two special cases: the first when $M$ consists solely of intervals, and the second when $M$ consists solely of circles.

If $M$ is a union of intervals, consider the following subset $\Gamma \subset \mathbb{X}_{\Sigma}$ of the 1-skeleton of the definition complex of $\Sigma:=\Sigma_{1} \cup_{M} \Sigma_{2}$. The vertices of $\Gamma$ are the soccer ball decompositions $X$ with the property that each connected component of $M \subset \Sigma$ is a single edge of $X$, and an edge $X \stackrel{\mathbb{D}}{\sim} Y$ is in $\Gamma$ if either $\mathbb{D} \subset \Sigma_{1}$ or $\mathbb{D} \subset \Sigma_{2}$. Note that $\Gamma$ is a connected graph. Note also that if a soccer ball decomposition $X$ belongs to $\Gamma$, then all the 2-cells of $X$, that are adjacent to $M$, are white. Given $X \in \Gamma$, let us write $\left.X\right|_{\Sigma_{1}}$ and $\left.X\right|_{\Sigma_{2}}$ for the restrictions of the soccer ball decomposition $X$ to $\Sigma_{1}$ and to $\Sigma_{2}$. For every $X \in \Gamma$, there is then an obvious isomorphism

$$
g_{X}: V(\Sigma ; X) \rightarrow V\left(\Sigma_{1} ;\left.X\right|_{\Sigma_{1}}\right) \bigotimes_{\mathcal{A}(M)} V\left(\Sigma_{2} ;\left.X\right|_{\Sigma_{2}}\right),
$$

well defined up to phase. Moreover, for every edge $X \stackrel{\mathbb{D}}{\sim} Y$ of $\Gamma$, the diagram

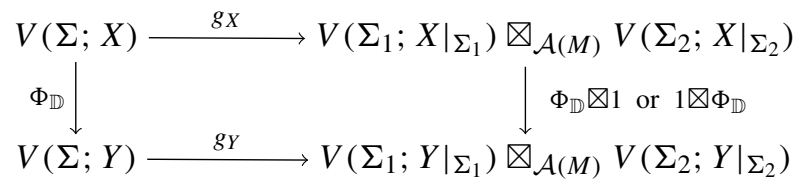

commutes up to phase, where $\Phi_{\mathbb{D}}$ are as in (2.17). Since $\Gamma$ is connected, it follows from (2.37) that the maps $g_{X}$ for $X \in \Gamma$ descend to a unitary isomorphism 


$$
g: V(\Sigma) \rightarrow V\left(\Sigma_{1}\right) \otimes_{\mathcal{A}(M)} V\left(\Sigma_{2}\right)
$$

well defined up to phase.

If $M$ is a union of circles, let us define the auxiliary surfaces

$$
\begin{aligned}
& \Sigma_{1}^{+}:=\Sigma_{1} \cup_{M}(M \times[0,1]), \quad \Sigma_{2}^{+}:=(M \times[0,1]) \cup_{M} \Sigma_{2}, \\
& \Sigma^{+}:=\Sigma_{1} \cup_{M}(M \times[0,1]) \cup_{M} \Sigma_{2} .
\end{aligned}
$$

Recall the definition of 2MAN from (2.20), and note that there are canonical isomorphisms $\Sigma_{1}^{+} \cong \Sigma_{1}, \Sigma_{2}^{+} \cong \Sigma_{2}, \Sigma^{+} \cong \Sigma$ in that groupoid (even though there are no canonical homeomorphisms). By Theorem 2.18, we therefore have unitary isomorphisms $V\left(\Sigma_{1}^{+}\right) \cong V\left(\Sigma_{1}\right), V\left(\Sigma_{2}^{+}\right) \cong V\left(\Sigma_{2}\right), V\left(\Sigma^{+}\right) \cong V(\Sigma)$, well defined up to phase. Therefore, instead of (2.30), we may as well construct an isomorphism

$$
g: V\left(\Sigma^{+}\right) \rightarrow V\left(\Sigma_{1}^{+}\right) \bigotimes_{\mathcal{A}(M)} V\left(\Sigma_{2}^{+}\right) .
$$

Given open soccer ball decompositions $X$ of $\Sigma_{1}$ and $Y$ of $\Sigma_{2}$, we let (2.38) be the composite

$$
\begin{aligned}
V\left(\Sigma^{+}\right) & \rightarrow X \triangleleft V(M \times[0,1]) \triangleright Y \\
& \rightarrow X \triangleleft L^{2} \mathcal{A}(M) \triangleright Y \\
& \rightarrow X \triangleleft L^{2} \mathcal{A}(M) \bigotimes_{\mathcal{A}(M)} L^{2} \mathcal{A}(M) \triangleright Y \\
& \rightarrow X \triangleleft V(M \times[0,1]) \bigotimes_{\mathcal{A}(M)} V(M \times[0,1]) \triangleright Y \\
& \rightarrow V\left(\Sigma_{1}^{+}\right) \bigotimes_{\mathcal{A}(M)} V\left(\Sigma_{2}^{+}\right)
\end{aligned}
$$

where the first map is $\lambda_{X \sqcup Y}$ from (2.36), the second and fourth maps are provided by (1.19), established in Theorem B.17, and the last map is the inverse of $\lambda_{X} \otimes \lambda_{Y}$. We need to show that the above map does not depend on $X$ and on $Y$. The following diagram is easily seen to commutate up to phase:

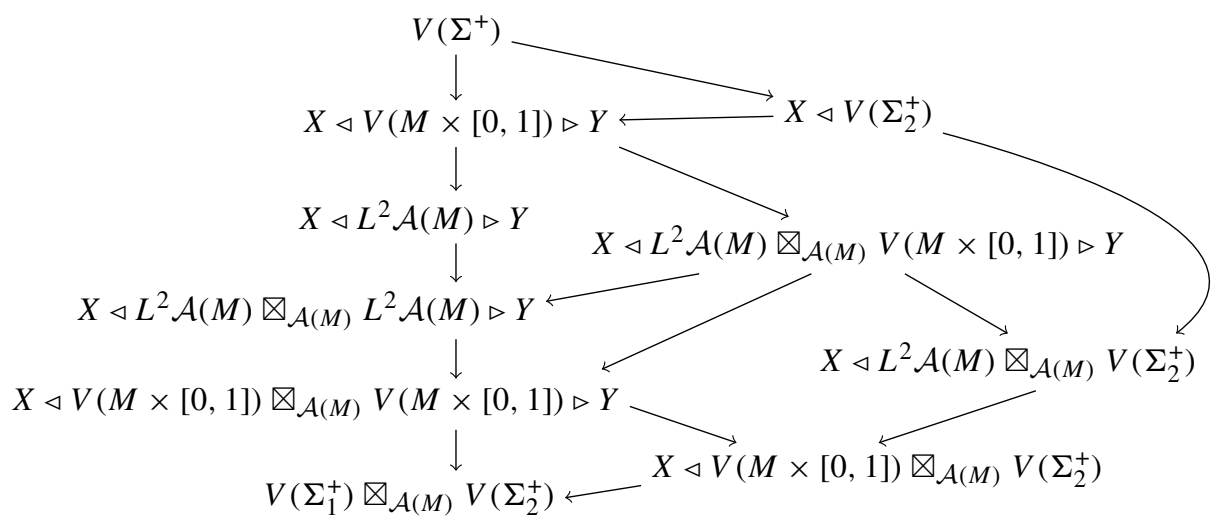

The vertical map from $V\left(\Sigma^{+}\right)$to $V\left(\Sigma_{1}^{+}\right) \bigotimes_{\mathcal{A}(M)} V\left(\Sigma_{2}^{+}\right)$is our definition of the map $g$ (2.38), and it is equal to the rightmost composition, up to phase. The map $g$ therefore does not depend on $Y$, up to phase. Since $X$ and $Y$ enter symmetrically in the definition of $g$, the map is also independent of $X$.

We now treat the general case. Write $M=M_{1} \sqcup M_{2}$ as a disjoint union, where $M_{1}$ consists only of intervals, and $M_{2}$ consists only of circles. Since $\mathcal{A}\left(M_{2}\right)$ is a direct sum 
of type $I$ factors, $L^{2} \mathcal{A}\left(M_{2}\right)$ is an $\mathcal{A}\left(M_{2}\right) \bar{\otimes} \mathcal{A}\left(M_{2}\right)^{o p}$-module and we have canonical isomorphisms

$$
\begin{aligned}
V\left(\Sigma_{1}\right) \otimes_{\mathcal{A}(M)} V\left(\Sigma_{2}\right) & \cong V\left(\Sigma_{1}\right) \otimes_{\mathcal{A}\left(M_{1}\right) \bar{\otimes} \mathcal{A}\left(M_{2}\right)} V\left(\Sigma_{2}\right) \\
& \cong\left(V\left(\Sigma_{1}\right) \bigotimes_{\mathcal{A}\left(M_{1}\right)} V\left(\Sigma_{2}\right)\right) \otimes_{\mathcal{A}\left(M_{2}\right) \bar{\otimes} \mathcal{A}\left(M_{2}\right)^{o p}} L^{2} \mathcal{A}\left(M_{2}\right) \\
& \cong\left(V\left(\Sigma_{1}\right) \bigotimes_{\mathcal{A}\left(M_{1}\right)} V\left(\Sigma_{2}\right)\right) \otimes_{\mathcal{A}\left(M_{2}\right) \bar{\otimes} \mathcal{A}\left(M_{2}\right)^{o p}} V\left(M_{2} \times[0,1]\right),
\end{aligned}
$$

where the last equality follows from (1.19), that is Theorem B.17.

By the two special cases that we treated earlier, we have further isomorphisms

$$
\begin{aligned}
& \cong V\left(\Sigma_{1} \cup_{M_{1}} \Sigma_{2}\right) \otimes_{\mathcal{A}\left(M_{2}\right) \bar{\otimes} \mathcal{A}\left(M_{2}\right)^{o p}} V\left(M_{2} \times[0,1]\right) \\
& \cong V\left(\left(\Sigma_{1} \cup_{M_{1}} \Sigma_{2}\right) \cup_{M_{2} \sqcup \bar{M}_{2}}\left(M_{2} \times[0,1]\right)\right) .
\end{aligned}
$$

There is a canonical isomorphism in 2MAN between $\Sigma^{+}:=\left(\Sigma_{1} \cup_{M_{1}} \Sigma_{2}\right) \cup_{M_{2} \sqcup \bar{M}_{2}}\left(M_{2} \times\right.$ $[0,1])$ and $\Sigma:=\Sigma_{1} \cup_{M} \Sigma_{2}$, which by Theorem 2.18 translates to a unitary isomorphism $V\left(\Sigma^{+}\right) \cong V(\Sigma)$, canonical up to phase. Composing all the above maps, we obtain our desired unitary isomorphism $g: V(\Sigma) \rightarrow V\left(\Sigma_{1}\right) \bigotimes_{\mathcal{A}(M)} V\left(\Sigma_{2}\right)$, canonically up to phase.

The gluing isomorphisms (2.30) satisfy the following version of associativity. Let $\Sigma_{1}, \Sigma_{2}, \Sigma_{3}$ be surfaces, $M_{1} \subset \partial \Sigma_{1}, M \subset \partial \Sigma_{2}, N_{2} \subset \partial \Sigma_{2}, N \subset \partial \Sigma_{3}$ submanifolds with $M$ and $N_{2}$ disjoint, and let $M_{1} \rightarrow M$ and $N_{2} \rightarrow N$ be orientation reversing diffeomorphisms. Pick a smooth structure on the trivalent graph $\partial \Sigma_{1} \cup_{M} \partial \Sigma_{2} \cup_{N} \partial \Sigma_{3}$, compatibly with those of $\partial \Sigma_{i}$. Then the following diagram commutes up to phase:

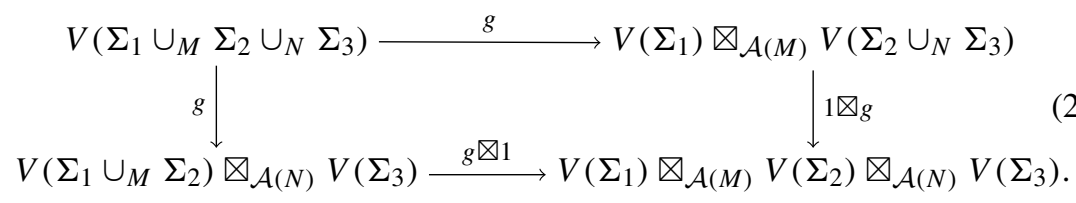

Indeed, our definition of the gluing isomorphism is local, and two gluings that happen far away do not interfere with each other.

Remark 2.41. As a consequence of Theorem 2.29, the definition of $V(\Sigma)$ presented in Sect. 2.B agrees with the more canonical one provided by Theorem 2.18 (which is based on (2.12) and (2.17)).

2.F. Conformal blocks. Recall that a "pair-of-pants" is just another name for a sphere with three holes, and that a pair-of-pants decomposition of a surface $\Sigma$ consists in a collection of circles in its interior that decompose it into pairs-of-pants $P_{1}, \ldots, P_{k}$ (we allow the situation where two legs of the same pair-of-pants get glued to each other).

With Theorem 2.29 in hand, it is now easy to compute the value of $V(\Sigma)$ for any surface $\Sigma$. Recall that we always assume our conformal net $\mathcal{A}$ has finite index, and the set $\Delta$ of isomorphism classes of irreducible $\mathcal{A}$-sectors is finite. Recall also that for $\lambda, \mu, v \in \Delta$, the multiplicity of $H_{0}$ inside $H_{\lambda} \otimes H_{\mu} \otimes H_{v}$ is denoted $N_{0}^{\lambda \mu \nu}$. 
Proposition 2.42. Let $\Sigma$ be a surface, with boundary components $S_{1}, \ldots, S_{n}$. Let $S_{n+1}$, $\ldots, S_{m}$ be oriented circles in the interior of $\Sigma$ that form a pairs-of-pants decomposition $P_{1}, \ldots, P_{k}$. Then the multiplicity of $H_{\lambda_{1}}\left(S_{1}\right) \otimes \cdots \otimes H_{\lambda_{n}}\left(S_{n}\right)$ in $V(\Sigma)$ is finite, and is given by

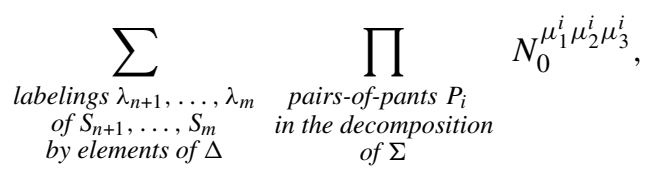

where $\mu_{1}^{i}, \mu_{2}^{i}, \mu_{3}^{i} \in \Delta$ are computed in the following way: letting $S_{j_{1}}, S_{j_{2}}, S_{j_{3}}$ be the three boundary component of $P_{i}$, then we set $\mu_{a}^{i}:=\lambda_{j_{a}}$ if the orientation $S_{j_{a}}$ is the one induced by $P_{i}$, and $\mu_{a}^{i}:=\bar{\lambda}_{j_{a}}$ otherwise.

Proof. For a manifold $M$, a Hilbert space $H$, and an isomorphism class of sector $\lambda \in \Delta$, let us write

$$
\begin{aligned}
M^{\epsilon} & :=\left\{\begin{array}{l}
M \text { for } \epsilon=+1 \\
\bar{M} \text { for } \epsilon=-1
\end{array}\right. \text { where the bar denotes orientation reversal, } \\
H^{\epsilon}: & =\left\{\begin{array}{l}
H \text { for } \epsilon=+1 \\
\bar{H} \text { for } \epsilon=-1
\end{array}\right. \text { where the bar denotes complex conjugate, } \\
\lambda^{\epsilon}: & =\left\{\begin{array}{l}
\lambda \text { for } \epsilon=+1 \\
\bar{\lambda} \text { for } \epsilon=-1
\end{array}\right. \text { where the bar denotes dual sector. }
\end{aligned}
$$

Let $\Sigma^{+}$be the surface that is obtained by gluing $A:=P_{1} \sqcup \cdots \sqcup P_{k}$ to $B:=\left(S_{n+1} \sqcup\right.$ $\left.\cdots \sqcup S_{m}\right) \times[0,1]$ along $\partial B$ in the obvious manner. There is a canonical isomorphism in 2MAN between $\Sigma$ and $\Sigma^{+}$, and so by Theorem 2.18 the spaces $V(\Sigma)$ and $V\left(\Sigma^{+}\right)$are isomorphic as $\partial \Sigma$-sectors.

For each pair-of-pants $P_{i}$ in the decomposition, let $j_{i 1}, j_{i 2}, j_{i 3} \in\{1, \ldots, m\}$ and $\epsilon_{i 1}, \epsilon_{i 2}, \epsilon_{i 3} \in\{ \pm 1\}$ be such that $\partial P_{i}=S_{j_{i 1}}^{\epsilon_{i 1}} \sqcup S_{j_{i 2}}^{\epsilon_{i 2}} \sqcup S_{j_{i 3}}^{\epsilon_{i 3}}$ as oriented manifolds. Recall that by Lemma 2.3 the Hilbert space associated to a pair-of-pants with boundary components $S_{1}, S_{2}, S_{3}$ is given by

$$
V(\bigcirc \bigcirc)=\bigoplus_{\lambda, \mu, v \in \Delta} N_{0}^{\lambda \mu v} H_{\lambda}\left(S_{1}\right) \otimes H_{\mu}\left(S_{2}\right) \otimes H_{v}\left(S_{3}\right)
$$

We therefore have

$$
\begin{aligned}
& V(A) \cong \bigotimes_{i=1}^{k} V\left(P_{i}\right) \cong \bigotimes_{i=1}^{k}\left(\bigoplus_{\lambda, \mu, v \in \Delta} N_{0}^{\lambda \mu \nu} H_{\lambda}\left(S_{j_{i 1}}^{\epsilon_{i 1}}\right) \otimes H_{\mu}\left(S_{j_{i 2}}^{\epsilon_{i 2}}\right) \otimes H_{\nu}\left(S_{j_{i 3}}^{\epsilon_{i 3}}\right)\right) \\
& \cong \bigotimes_{i=1}^{k}\left(\bigoplus_{\lambda, \mu, v \in \Delta} N_{0}^{\lambda \mu \nu}\left(H_{\lambda^{\epsilon} \epsilon_{1}}\left(S_{j_{i 1}}\right)\right)^{\epsilon_{i 1}} \otimes\left(H_{\mu^{\epsilon}{ }^{2}}\left(S_{j_{i 2}}\right)\right)^{\epsilon_{i 2}} \otimes\left(H_{\nu^{\epsilon_{i 3}}}\left(S_{j_{i 3}}\right)\right)^{\epsilon_{i 3}}\right) \\
& \cong \bigotimes_{i=1}^{k}\left(\bigoplus_{\lambda, \mu, v \in \Delta} N_{0}^{\lambda^{\epsilon_{i 1}} \mu^{\epsilon_{i 2} v_{i 3}}}\left(H_{\lambda}\left(S_{j_{i 1}}\right)\right)^{\epsilon_{i 1}} \otimes\left(H_{\mu}\left(S_{j_{i 2}}\right)\right)^{\epsilon_{i 2}} \otimes\left(H_{\nu}\left(S_{j_{i 3}}\right)\right)^{\epsilon_{i 3}}\right),
\end{aligned}
$$


where the middle isomorphism follows from [8, Lem. 3.17]. The multiplicity of

$$
\left.\begin{array}{rl}
\left(H_{\lambda_{1}}\left(S_{1}\right) \otimes \cdots \otimes H_{\lambda_{n}}\left(S_{n}\right)\right) \otimes\left(H_{\lambda_{n+1}}\left(S_{n+1}\right) \otimes \cdots \otimes H_{\lambda_{m}}\left(S_{m}\right)\right) & \\
\otimes\left(\overline{H_{\lambda_{n+1}^{\prime}}\left(S_{n+1}\right)} \otimes \cdots \otimes \overline{H_{\lambda_{m}^{\prime}}\left(S_{m}\right)}\right.
\end{array}\right)
$$

inside $V(A)$ is thus given by

$$
m_{\lambda_{1}, \ldots, \lambda_{n}, \lambda_{n+1}, \ldots, \lambda_{m}, \lambda_{n+1}^{\prime}, \ldots, \lambda_{m}^{\prime}}:=\prod_{i=1}^{k} N_{0}^{\nu_{1}^{i} v_{2}^{i} v_{3}^{i}}
$$

where $v_{a}^{i}$ is defined to be $\lambda_{j_{i a}}$ if the orientation of $S_{j_{i a}}$ is the one induced by $P_{i}$, and $\bar{\lambda}_{j i a}^{\prime}$ otherwise.

We have $V(B) \cong L^{2} \mathcal{A}\left(S_{n+1}\right) \otimes \cdots \otimes L^{2} \mathcal{A}\left(S_{m}\right)$ by (1.19), that is Theorem B.17, and so the operation $-\bigotimes_{\mathcal{A}(\partial B)} V(B)$ amounts to contracting the indices $\lambda_{i}$ with $\lambda_{i}^{\prime}$ for $i \in\{n+1, \ldots, m\}$. The multiplicity of $H_{\lambda_{1}}\left(S_{1}\right) \otimes \cdots \otimes H_{\lambda_{n}}\left(S_{n}\right)$ inside $V(\Sigma) \cong$ $V\left(\Sigma^{+}\right) \cong V(A) \bigotimes_{\mathcal{A}(\partial B)} V(B)$ is therefore given by

$$
\sum_{\left(\lambda_{n+1}, \ldots, \lambda_{m}\right) \in \Delta^{m-n}} m_{\lambda_{1}, \ldots, \lambda_{n}, \lambda_{n+1}, \ldots, \lambda_{m}, \lambda_{n+1}, \ldots, \lambda_{m},}
$$

which is precisely (2.43).

Note that for $\partial \Sigma=\emptyset$, the above result implies that $V(\Sigma)$ is a finite dimensional:

Corollary 2.44. Let $\Sigma$ be a closed surface and let $S_{1}, \ldots, S_{m}$ be oriented circles in $\Sigma$ that form a pair-of-pants decomposition $P_{1}, \ldots, P_{k}$. Then $V(\Sigma)$ is finite dimensional, and its dimension is given by

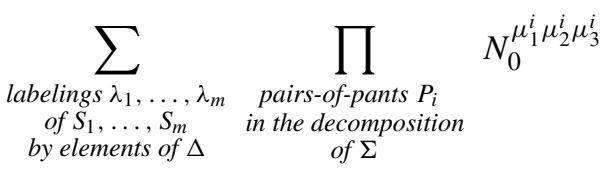

where $\mu_{1}^{i}, \mu_{2}^{i}, \mu_{3}^{i}$ are as defined in Proposition 2.42.

Given the formula (2.45), it now seems reasonable that for a closed surface $\Sigma$, our vector space $V(\Sigma)$ agrees with the notion of "space of conformal blocks" in conformal field theory [22,31]. Moreover, for a surface $\Sigma$ with boundary components $S_{1}, \ldots, S_{n}$, the vector space

$$
V\left(\Sigma ; \lambda_{1}, \ldots, \lambda_{n}\right):=\operatorname{hom}\left(H_{\lambda_{1}}\left(S_{1}\right) \otimes \cdots \otimes H_{\lambda_{n}}\left(S_{n}\right), V(\Sigma)\right)
$$

corresponds to the notion of "space of conformal blocks with field insertions". Note that those statements, relating our space of conformal blocks to the CFT notion of space of conformal blocks, are really only conjectures because, for example, there is no theorem that identifies the representation theory of a conformal net with that of the corresponding chiral conformal field theory.

For every choice of labels $\lambda_{1}, \ldots, \lambda_{n} \in \Delta$, there is a projective action of $\Gamma(\Sigma):=$ $\operatorname{ker}(G(\Sigma) \rightarrow \operatorname{Diff}(\partial \Sigma))$ on $V\left(\Sigma ; \lambda_{1}, \ldots, \lambda_{n}\right)$. Note however that the projective action of $G(\Sigma)$ on $V(\Sigma)$ contains strictly more information than the collection of all the projective actions of $\Gamma(\Sigma)$ on the spaces $V\left(\Sigma ; \lambda_{1}, \ldots, \lambda_{n}\right)$. 
Remark 2.47. If one only considers surfaces whose boundary components are parametrized by $S^{1}$, and if one restricts attention to those gluings that are along full boundary components and that are compatible with the parametrizations, then Theorems 2.18 and 2.29 provide a projective version of a modular functor (as in [6, Def. 5.7.10], but without any control over the central cocycle).

\section{Modularity}

In this section, we will use the constructions from the previous sections to analyze the structure of the category of sectors of a conformal net with finite index.

\section{A. Fusion of sectors. Let us define the standard pair-of-pants to be the surface}

$$
P:=\left(S^{1} \times[0,1]\right) \sqcup\left(S^{1} \times[0,1]\right) / \sim,
$$

where the equivalence relation identifies the point $(z, 1)$ in the first copy of $S^{1} \times[0,1]$ with the point $(-\bar{z}, 1)$ in the second copy of $S^{1} \times[0,1]$, for every $z \in S_{\vdash}^{1}=\left\{z \in S^{1} \mid \Re e(z) \leq\right.$ $0\}$. As mentioned at (1.6) and (1.19), and proven in Theorems B.13 and B.17, the Hilbert space $V\left(S^{1} \times[0,1]\right) \cong L^{2} \mathcal{A}\left(S^{1}\right)$ associated to $S^{1} \times[0,1]$ is well defined up to canonical unitary isomorphism, as opposed to being merely well defined up to canonical-up-tophase unitary isomorphism. We may therefore declare

$$
H_{P}:=L^{2} \mathcal{A}\left(S^{1}\right) \otimes_{\mathcal{A}\left(S_{\vdash}^{1}\right)} L^{2} \mathcal{A}\left(S^{1}\right)
$$

to be the Hilbert space associated to $P$. This is a lift of $V(P)$ from a Hilbert space well defined up to canonical-up-to-phase isomorphism to an honest Hilbert space.

Let us call $S_{1}, S_{2}, S_{3}$ the three boundary components of $P$, and orient them as indicated in the following picture:

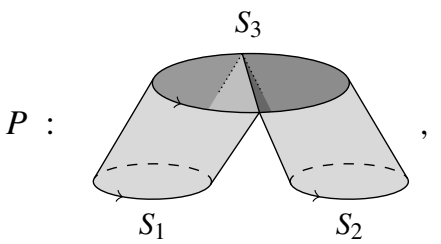

so that $\partial P=S_{1} \sqcup S_{2} \sqcup \bar{S}_{3}$. Below, we will use the picture for for that same manifold $P$.

Recall from $[8$, Sec. $1 . E]$ that there is a monoidal structure

$$
H, K \mapsto H \otimes^{\mathrm{h}} K:=H \otimes_{\mathcal{A}\left(S_{\vdash}^{1}\right)} K
$$

on the category $\operatorname{Sect}(\mathcal{A})$, called horizontal fusion. The $S_{1}-S_{2}-\bar{S}_{3}$-sector $H_{P}$ is constructed in such a way that there are canonical unitary isomorphisms

$$
\begin{aligned}
H \otimes^{\mathrm{h}} K & =H \otimes_{\mathcal{A}\left(S_{\vdash}^{1}\right)} K \\
& \cong\left(H \otimes_{\mathcal{A}\left(S_{1}\right)} L^{2} \mathcal{A}\left(S_{1}\right)\right) \otimes_{\mathcal{A}\left(S_{\vdash}^{1}\right)}\left(K \otimes_{\mathcal{A}\left(S_{2}\right)} L^{2} \mathcal{A}\left(S_{2}\right)\right) \\
& \cong(H \otimes K) \otimes_{\mathcal{A}\left(S_{1} \cup S_{2}\right)}\left(L^{2} \mathcal{A}\left(S_{1}\right) \otimes_{\mathcal{A}\left(S_{\vdash}^{1}\right)} L^{2} \mathcal{A}\left(S_{2}\right)\right) \\
& =(H \otimes K) \otimes_{\mathcal{A}\left(S_{1} \cup S_{2}\right)} H_{P} .
\end{aligned}
$$


In other words, $H_{P}$ represents the operation of horizontal fusion.

We will be interested in various ways of fusing copies of $H_{P}$ to each other. For $a, b \in\{1,2,3\}$, we shall write $H_{P}{ }_{a} \bigotimes_{b} H_{P}$ for the fusion $H_{P} \bigotimes_{\mathcal{A}\left(S^{1}\right)} H_{P}$, where the algebra $\mathcal{A}\left(S^{1}\right)$ acts on first copy of $H_{P}$ via its isomorphism to $\mathcal{A}\left(S_{a}\right)$ (or its opposite), and on the second $H_{P}$ via its isomorphism to $\mathcal{A}\left(S_{b}\right)$ (or its opposite). For example, the Hilbert spaces $H_{P}{ }_{3} \bigotimes_{1} H_{P}$ and $H_{P}{ }_{2} \bigotimes_{3} H_{P}$ correspond to the following two surfaces:
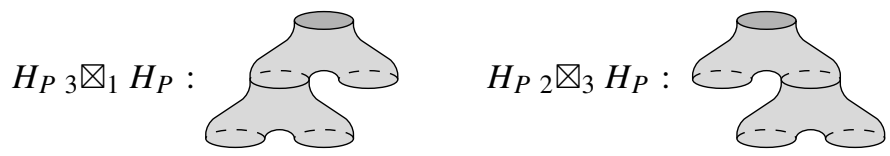

Note that the associator for $\otimes^{\mathrm{h}}$ corresponds to a particular unitary isomorphism

$$
\alpha: H_{P}{ }_{3} \bigotimes_{1} H_{P} \rightarrow H_{P}{ }_{2} \bigotimes_{3} H_{P}
$$

namely the one given by

$$
\begin{aligned}
& H_{P}{ }_{3} \bigotimes_{1} H_{P}=\left(L^{2} \mathcal{A}\left(S^{1}\right) \bigotimes_{\mathcal{A}\left(S_{\vdash}^{1}\right)} L^{2} \mathcal{A}\left(S^{1}\right)\right){ }_{3} \bigotimes_{1}\left(L^{2} \mathcal{A}\left(S^{1}\right) \bigotimes_{\mathcal{A}\left(S_{\vdash}^{1}\right)} L^{2} \mathcal{A}\left(S^{1}\right)\right) \\
& =\left(L^{2} \mathcal{A}\left(S^{1}\right) \bigotimes_{\mathcal{A}\left(S_{\vdash}^{1}\right)} L^{2} \mathcal{A}\left(S^{1}\right)\right) \bigotimes_{\mathcal{A}\left(S^{1}\right)} L^{2} \mathcal{A}\left(S^{1}\right) \bigotimes_{\mathcal{A}\left(S_{\vdash}^{1}\right)} L^{2} \mathcal{A}\left(S^{1}\right) \\
& \rightarrow L^{2} \mathcal{A}\left(S^{1}\right) \otimes_{\mathcal{A}\left(S_{\vdash}^{1}\right)} L^{2} \mathcal{A}\left(S^{1}\right) \otimes_{\mathcal{A}\left(S_{\vdash}^{1}\right)} L^{2} \mathcal{A}\left(S^{1}\right) \\
& \rightarrow L^{2} \mathcal{A}\left(S^{1}\right) \otimes_{\mathcal{A}\left(S_{\vdash}^{1}\right)} L^{2} \mathcal{A}\left(S^{1}\right) \otimes_{\mathcal{A}\left(S^{1}\right)^{o p}}\left(L^{2} \mathcal{A}\left(S^{1}\right) \otimes_{\mathcal{A}\left(S_{\vdash}^{1}\right)} L^{2} \mathcal{A}\left(S^{1}\right)\right) \\
& =\left(L^{2} \mathcal{A}\left(S^{1}\right) \bigotimes_{\mathcal{A}\left(S_{\vdash}^{1}\right)} L^{2} \mathcal{A}\left(S^{1}\right)\right)_{2} \bigotimes_{3}\left(L^{2} \mathcal{A}\left(S^{1}\right) \otimes_{\mathcal{A}\left(S_{\vdash}^{1}\right)} L^{2} \mathcal{A}\left(S^{1}\right)\right) \\
& =H_{P}{ }_{2} \bigotimes_{3} H_{P} \text {. }
\end{aligned}
$$

This definition immediately raises a question. Since $H_{P}$ is a representative of the "up-to-phase equivalence class" $V(P)$, how does the above map $\alpha$ compare to the geometrically defined associator $\alpha_{g e o}: V(P)_{3} \bigotimes_{1} V(P) \rightarrow V(P)_{2} \bigotimes_{3} V(P)$

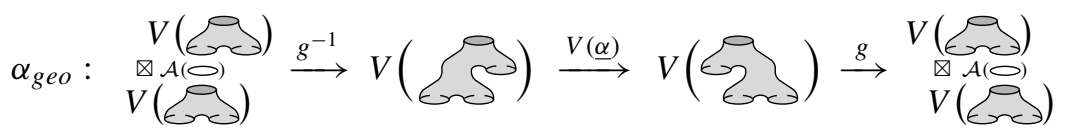

obtained by composing two gluing isomorphisms (2.30) and the image $V(\underline{\alpha})$ of the obvious diffeomorphism $\underline{\alpha}: S \stackrel{-}{\longrightarrow} \underset{S}{\longrightarrow}$ under the functor $V$ ? Since $\alpha_{\text {geo }}$ is only well defined up to phase, the best we can hope for is that the equation $\alpha_{g e o}=\alpha$ holds up to phase:

Proposition 3.2. The two maps $\alpha, \alpha_{g e o}: H_{P}{ }_{3} \bigotimes_{1} H_{P} \rightarrow H_{P}{ }_{2} \bigotimes_{3} H_{P}$ are equal up to a phase.

Before embarking on the proof, we introduce the following graphical notation (and, implicitly, obvious variations thereof): 


$$
\begin{aligned}
& L^{2}:=L^{2} \mathcal{A}\left(S^{1}\right) \\
& \underline{V}:=V(\Theta)=V\left(S^{1} \times[0,1]\right) \\
& \overbrace{L^{2} L^{2}}:=\underbrace{}_{L^{2}\left(S_{\vdash}^{1}\right)} \underbrace{}_{L^{2}}=H_{P} \\
& \overbrace{V}:=\underline{V} \otimes_{\mathcal{A}\left(S_{\vdash}\right)}, \underline{V} \\
& \overbrace{V}:=V\left(\Theta \cup_{S_{\vdash}^{1}} \boxminus\right)=V(P)
\end{aligned}
$$

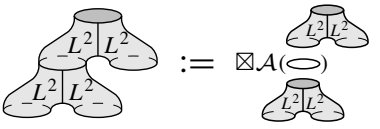

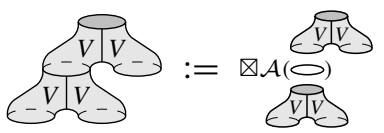

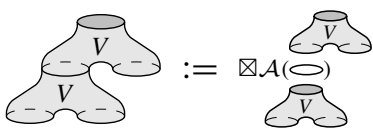

$$
\begin{aligned}
& \int_{V}:=V(\underset{S}{S})=V\left(P_{3} \cup_{1} P\right)
\end{aligned}
$$

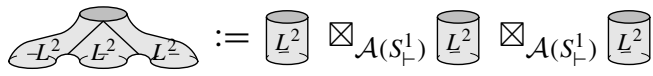

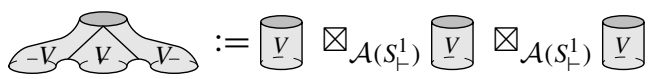

$$
\begin{aligned}
& \overbrace{V}:=V\left(\Xi \cup_{S_{\vdash}^{1}} \boxminus \cup_{S_{\vdash}^{1}} \boxminus\right)
\end{aligned}
$$

Proof of Proposition 3.2. Keeping in mind that the identification between $H_{P}$ and $V(P)$ is given by

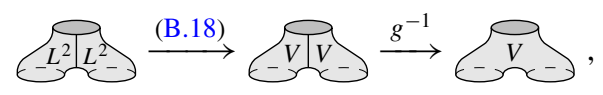


our task is to prove the commutativity of this diagram:

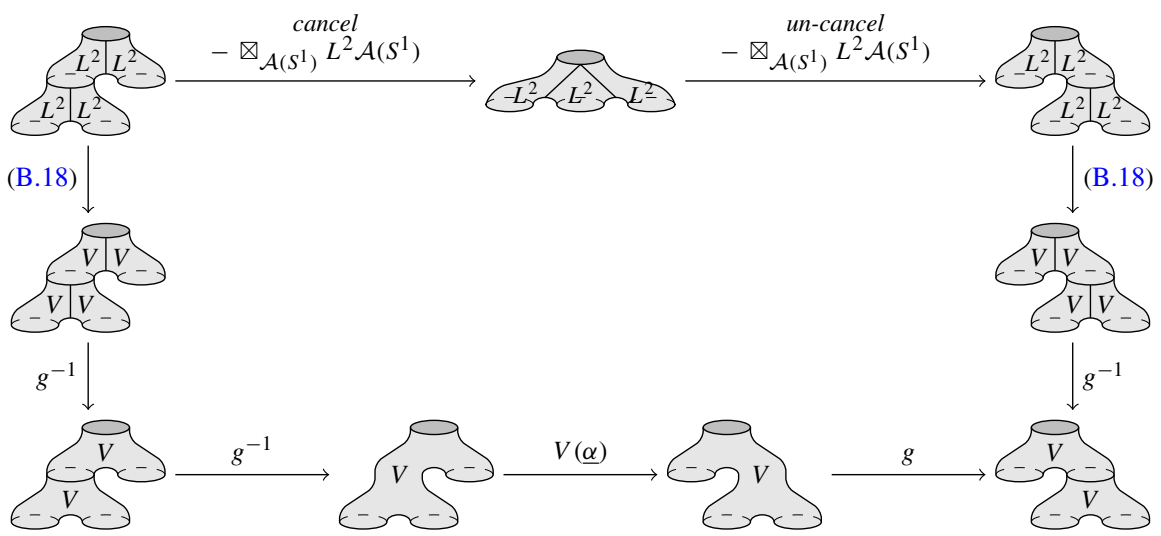

Note that the top composite is $\alpha$ and that the bottom composite is $\alpha_{g e o}$.

We can split this square into two mirror-image sub-diagrams by inserting

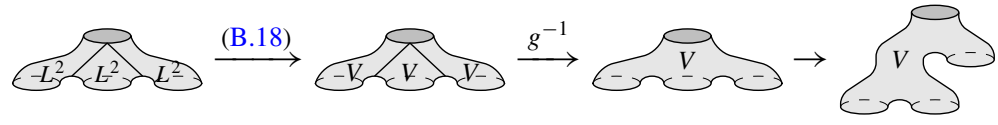

down the middle (where the last map is induced by the obvious diffeomorphism and there is an analogous map to the target of $V(\underline{\alpha})$ ). It is therefore enough to prove the commutativity of the following 7-gon:

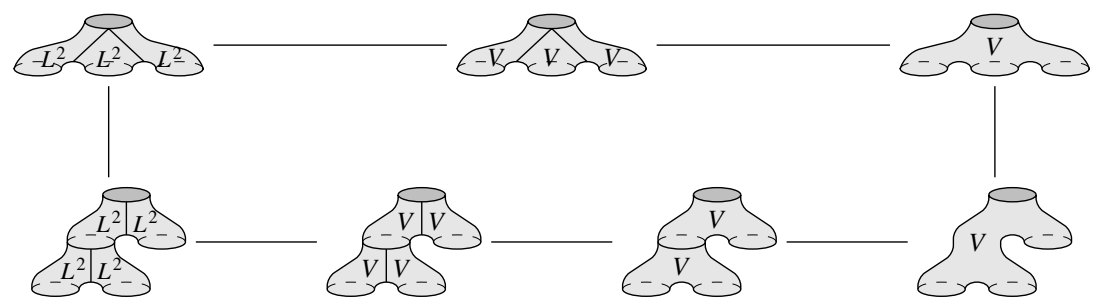

Let $X$ be an $\left(S^{1} \times\{0\}\right)$-open soccer ball decomposition of $S^{1} \times[0,1]$. We will prove that (3.3) is commutative after applying the invertible functor $X \triangleleft-$. Namely, we will prove the commutativity of this diagram:

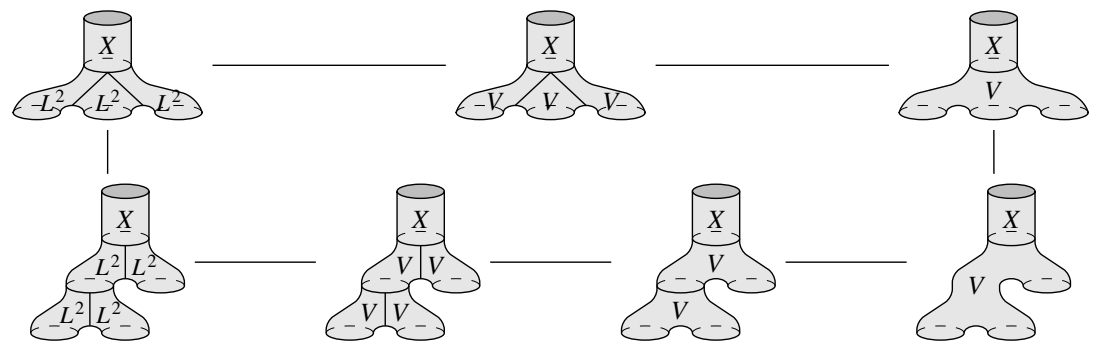


Note that the above pictures are somewhat misleading: these constructions involve not just a single Connes fusion over the bottom circle of the annulus labeled $X$, but rather many fusions, each one of which uses at most an interval in this circle.

By choosing $X$ carefully, we can arrange that the union of $X$ and of a soccer ball decomposition of $S^{1} \times[0,1]$ (which again needs to be picked carefully, namely so that the left half of the top $S^{1}$ is covered by a single edge) is an open soccer ball decomposition, call it $Y$, of the manifold

$$
\sum_{-\rightarrow}=\left(S^{1} \times[0,1]\right) \sqcup\left(S^{1} \times[0,1]\right) / \sim,
$$

where the equivalence relation identifies $(z, 0)$ in the first copy of $S^{1} \times[0,1]$ with $(z, 1)$ in the second copy of $S^{1} \times[0,1]$, for every $z \in S_{\dashv}^{1}$.

With these preliminaries in place, the commutativity of (3.4) follows from this commutative diagram:

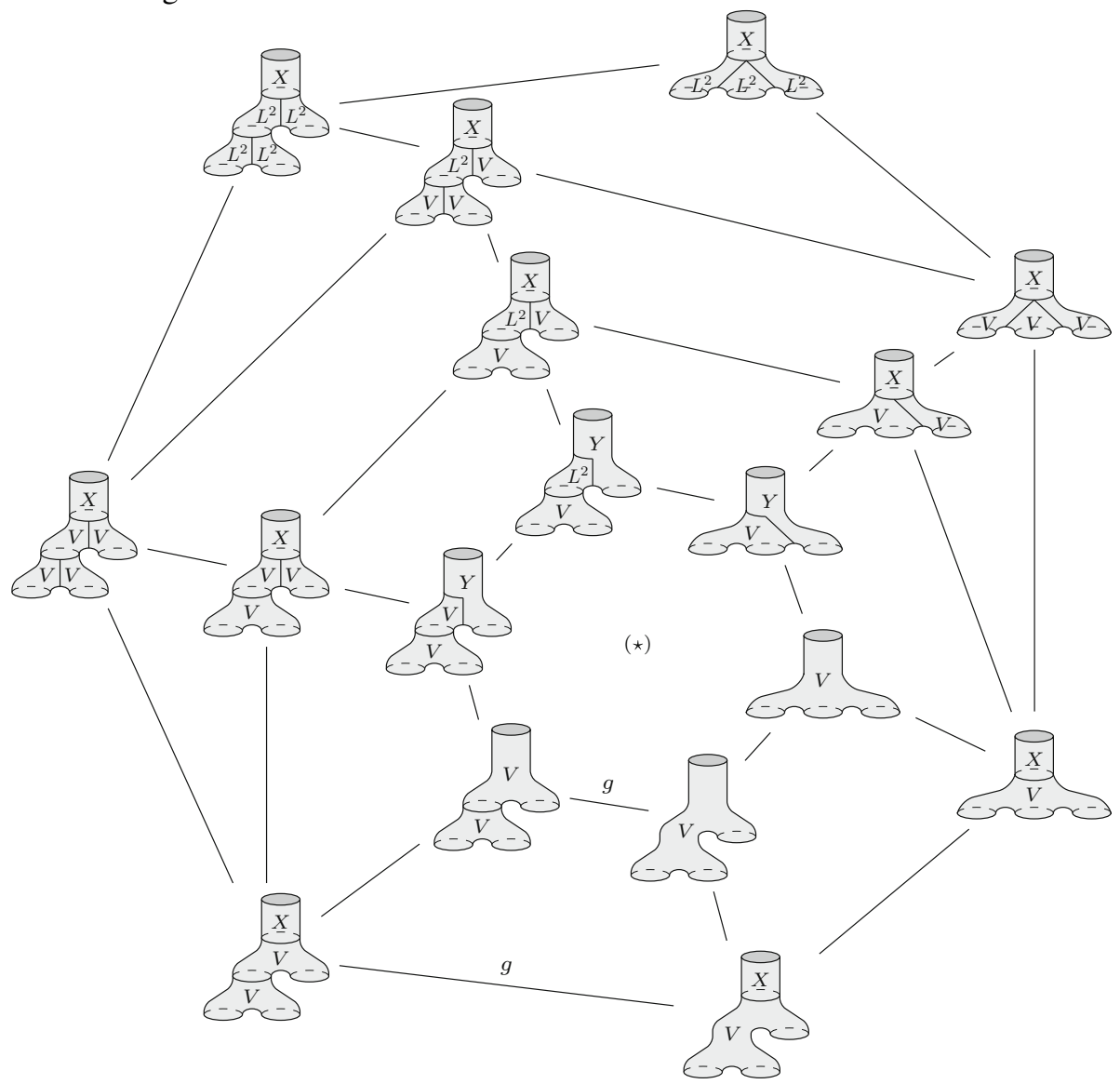

Here, the central hexagon $(\star)$ commutes because the gluing isomorphism $g$ is equal to the rightmost composition in (2.39), precomposed by the diffeormorphism ${ }_{V} \cong$ S 
3.B. The braiding. The extended mapping class group of the standard pair-of-pants contains a special element $\underline{\beta}: P \rightarrow P$, called the braiding, that exchanges the two boundary circles $S_{1}$ and $S_{2}$, and fixes $S_{3}$. Using a flat depiction of $P$, we illustrate the homeomorphism $\underline{\beta}$ by means of what it does to some internal lines:
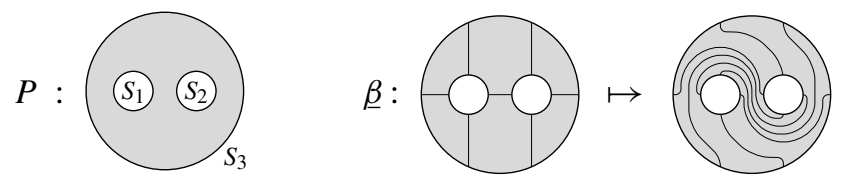

By Theorem 2.18, there is an associated unitary

$$
\beta:=V(\underline{\beta}): H_{P} \rightarrow H_{P},
$$

well defined up to phase. Moreover, letting $\tau: H_{0} \otimes H_{0} \rightarrow H_{0} \otimes H_{0}$ be the switch map, we can fix the phase of $\beta$ by requiring that the composite

$$
\begin{aligned}
H_{0} \cong H_{0} \nabla^{\mathrm{h}} H_{0} & \cong\left(H_{0} \otimes H_{0}\right) \otimes_{\mathcal{A}\left(S_{1} \cup S_{2}\right)} H_{P} \\
& \stackrel{\tau \otimes \beta}{\longrightarrow}\left(H_{0} \otimes H_{0}\right) \bigotimes_{\mathcal{A}\left(S_{1} \cup S_{2}\right)} H_{P} \cong H_{0} \nabla^{\mathrm{h}} H_{0} \cong H_{0}
\end{aligned}
$$

be the identity on $H_{0}$.

Theorem 3.7. The transformation

$$
\begin{aligned}
\beta_{H, K}: H \otimes^{\mathrm{h}} K & \cong(H \otimes K) \bigotimes_{\mathcal{A}\left(S_{1} \cup S_{2}\right)} H_{P} \\
& \stackrel{\tau \otimes \beta}{\longrightarrow}(K \otimes H) \bigotimes_{\mathcal{A}\left(S_{1} \cup S_{2}\right)} H_{P} \cong K \nabla^{\mathrm{h}} H
\end{aligned}
$$

equips the category $\left(\operatorname{Sect}(\mathcal{A}), \nabla^{\mathrm{h}}\right)$ with the structure of a braided tensor category.

Proof. We need to show that the isomorphisms $\beta_{H, K}$ are natural in $H$ and $K$, and that they satisfy the two hexagon axioms. Naturality is obvious from the definition. The two hexagon axioms are

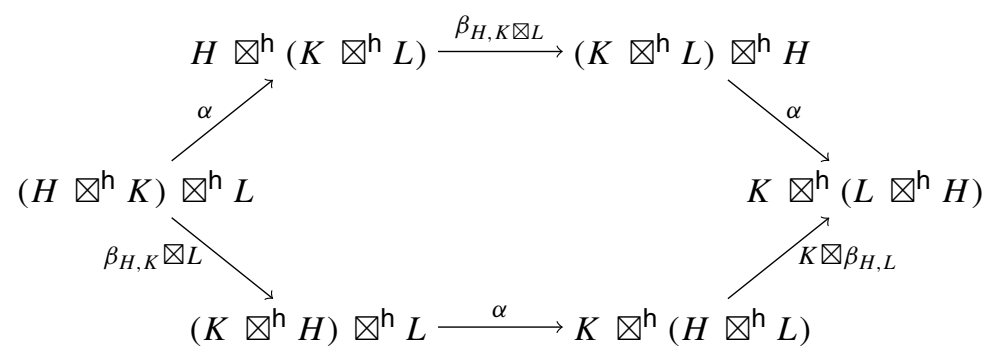

and

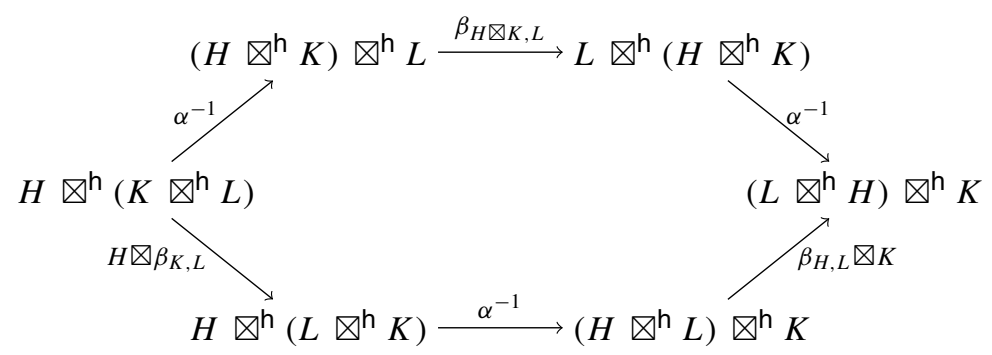


and we shall only treat the first one here.

The following diagram is commutative in the groupoid $2 \mathrm{MAN}$

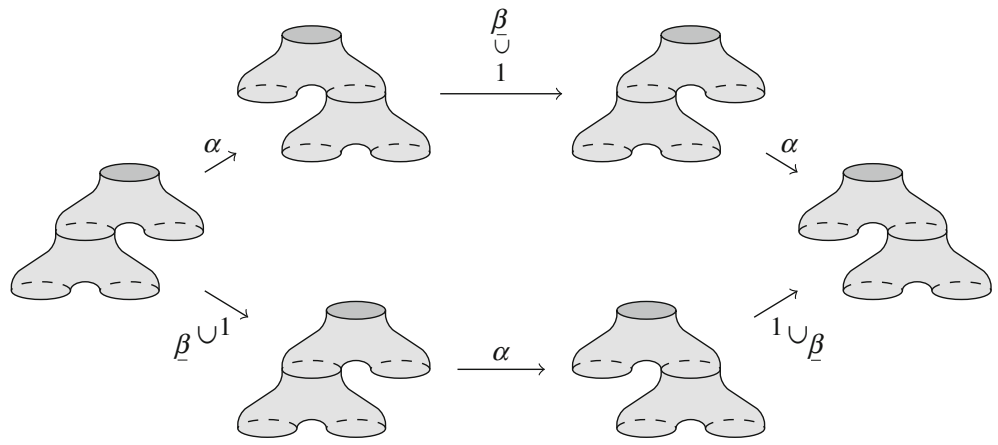

By Theorem 2.18 and Proposition 3.2, the corresponding diagram of Hilbert spaces

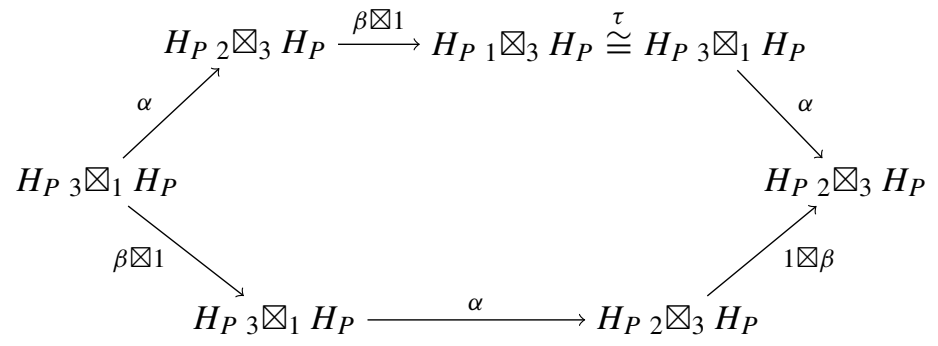

therefore commutes up to phase. If we fuse $H_{0} \otimes H_{0} \otimes H_{0}$ onto (3.8), then the resulting diagram

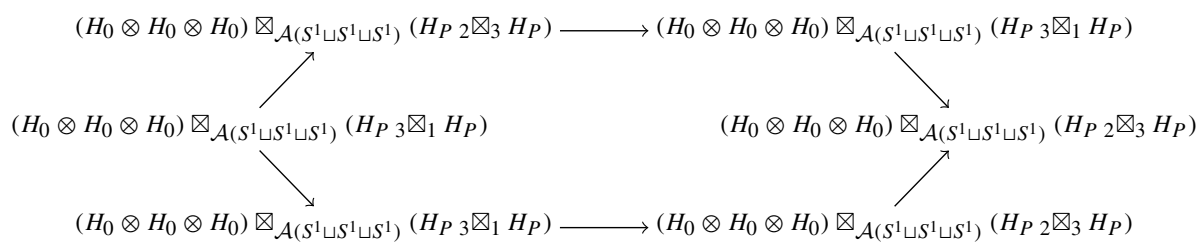

is simply

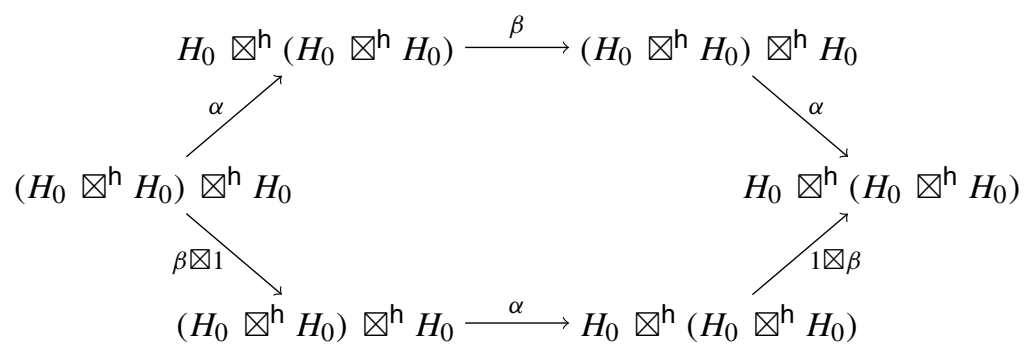

and it follows from (3.6) that it is commutative, not just up to phase. The diagram (3.8) was therefore also commutative, not just up to phase.

It would be interesting to compare the braiding $\beta$ defined above with the one introduced in [23]. We conjecture that those two braidings are equal. 
3.C. Modularity of the category of sectors. Based on the technology that we developed in the previous sections, we will now present a new proof of a famous result of Kawahigashi-Longo-Müger [26, Cor. 37] about the modularity of the representation category of conformal nets.

Recall that an object $T$ in a braided tensor category is called transparent if the equality

$$
\beta_{X, T}=\beta_{T, X}^{-1}: X \otimes T \rightarrow T \otimes X
$$

holds for every object $X$ in the category. By Müger's theorem (first proven by Rehren in [36] in the language of low-dimensional algebraic quantum field theory and then by Müger in an abstract categorical setup [33, Cor. 7.11] — see also [12] for a proof that does not require unitarity), one of the equivalent definitions of a modular tensor category is a braided fusion category in which the only transparent objects are the multiples of the identity.

Theorem 3.9. Let $\mathcal{A}$ be a conformal net with finite index. Then the braided tensor category $\operatorname{Sect}(\mathcal{A})$ is modular.

Remark 3.10. As explained in Remark 2.47, the system of spaces of conformal blocks we have constructed together with the factorization property has as a special case the data of the projective version of a modular functor. If we knew that the central extension of the bordism 2-category of surfaces with parametrized boundary (objects=unions of circles, morphisms=cobordisms, 2-morphisms=homeomorphisms modulo isotopy rel boundary) described in [6, Def. 5.7.6] is a universal central extension, then we could deduce that we have a modular functor in the sense of [6, Def. 5.7.10]. The results of Bakalov-Kirillov [6, Thm. 5.7.11] would then provide yet another proof of the above modularity theorem.

Proof. Given an irreducible transparent object $T \in \operatorname{Sect}(\mathcal{A})$, we need to show that $T$ is isomorphic to $H_{0}$.

We shall draw the standard pair of pants $P$ as in (3.5). Consider the following submanifolds of $P$

$A:$

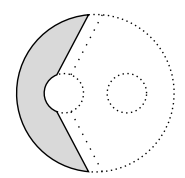

$B:$

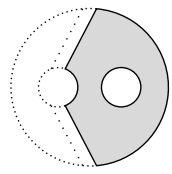

C:

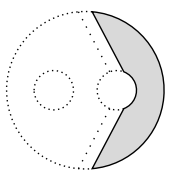

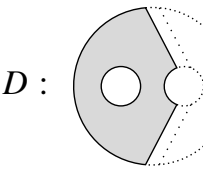

and let $i_{A}: A \hookrightarrow P, \ldots, i_{D}: D \hookrightarrow P$ be the inclusion maps.

Let us also consider the manifolds

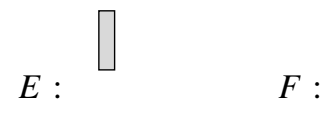

along with embeddings $f, g: E \rightarrow P$ and $h, k: F \rightarrow P$

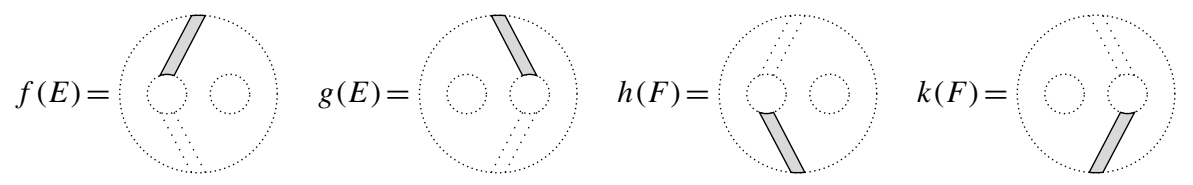


whose images in $P$ are such that

$$
A \cup f(F) \cup B \cup h(E)=P \quad \text { and } \quad C \cup g(E) \cup D \cup k(F)=P .
$$

Recall from Theorem 2.18 that $V(A), \ldots, V(F)$ are only well defined up to canonicalup-to-phase unitary isomorphism. Let us fix, by means of some arbitrary choice, Hilbert spaces $V(A), \ldots, V(F)$ within their "up-to-phase isomorphism class".

Let us write $V(\bigcirc \circ)$ ) for the Hilbert space $H_{P}$ defined in (3.1). By Theorem 2.29, there are unitary isomorphisms

$$
\begin{aligned}
& \kappa_{l}:, \quad V(A) \otimes V(F) \otimes V(B) \otimes V(E) \otimes-; \rightarrow V(\bigcirc \bigcirc) \\
& \left.\kappa_{r}: \underset{i}{i} V(C) \otimes V(E) \otimes V(D) \otimes V(F) \otimes-, \rightarrow V(\bigcirc \bigcirc)\right)
\end{aligned}
$$

that correspond to the homeomorphisms

$$
\begin{array}{ll}
i_{A} \cup h \cup i_{B} \cup f: & A \cup F \cup B \cup E \rightarrow P \\
i_{C} \cup g \cup i_{D} \cup k: & C \cup E \cup D \cup F \rightarrow P,
\end{array}
$$

(the algebras over which the fusion product are taken are omitted from the notation), and that are well defined up to phase. Fix choices of $\kappa_{l}$ and $\kappa_{r}$ within their equivalence classes.

Recall the definition of $\underline{\beta}: P \rightarrow P$ from (3.5), and let
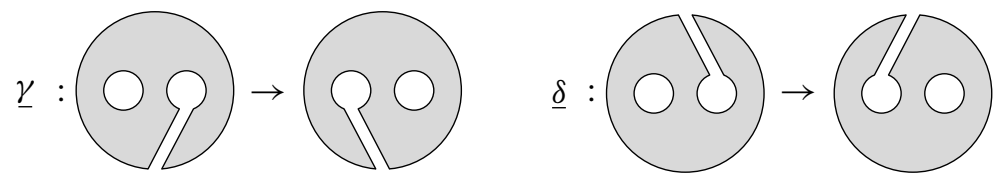

be homeomorphisms such that $\underline{\gamma} \cup\left(h \circ k^{-1}\right)=\underline{\beta}$ and $\underline{\delta} \cup\left(f \circ g^{-1}\right)=\underline{\beta}^{-1}$ in the groupoid 2MAN (assume that $f, g, h, k$ are chosen so that this is possible). Let then

$$
\begin{aligned}
& \gamma: V(C) \otimes V(E) \otimes V(D) \rightarrow V(B) \otimes V(E) \otimes V(A) \\
& \delta: V(D) \otimes V(F) \otimes V(C) \rightarrow V(A) \otimes V(F) \otimes V(B)
\end{aligned}
$$

be the unique representatives $\gamma \in V(\underline{\gamma}), \delta \in V(\underline{\delta})$ for which

; $\gamma \otimes 1_{V(F)} \otimes_{-;}=\kappa_{l}^{-1} \circ \beta \circ \kappa_{r} \quad$ and $\quad,-\delta \otimes 1_{V(E)} \otimes_{-;}=\kappa_{l}^{-1} \circ \beta^{-1} \circ \kappa_{r}$.

Let us agree that $V(\bigcirc)), V(\Omega 0), V(\circ)), V(B \circ))$ stand for the Hilbert spaces $V(C) \otimes V(E) \otimes V(D), V(B) \otimes V(E) \otimes V(A), V(D) \otimes V(F) \otimes V(C)$, and $V(A) \otimes$ $V(F) \otimes V(B)$, so we can rewrite (3.11) as

$$
\gamma: V(\bigcirc \Omega)) \rightarrow V(\Omega \circ), \quad \delta: V(O \delta)) \rightarrow V(B O)) .
$$

Let now $T \in \operatorname{Sect}(\mathcal{A})$ be irreducible and transparent. By definition of transparent, the two natural transformations $\beta_{T,-}$ and $\beta_{-, T}^{-1}$ from $T \nabla^{\mathrm{h}}-$ to $-\nabla^{\mathrm{h}} T$ are equal to 
each other. Equivalently, the two maps $1_{T} \otimes \beta$ and $1_{T} \otimes \beta^{-1}$ from $T \otimes_{\mathcal{A}\left(S_{1}\right)} H_{P}$ to $T \otimes_{\mathcal{A}\left(S_{2}\right)} H_{P}$ are equal to each other. Let us define

$$
\begin{aligned}
& \beta_{T}:=1_{T} \otimes \beta=1_{T} \otimes \beta^{-1}: \quad T \otimes_{\mathcal{A}\left(S_{1}\right)} V(\bigcirc \mathrm{O}) \rightarrow T \otimes_{\mathcal{A}\left(S_{2}\right)} V(\bigcirc \circ) \\
& \left.\left.\gamma_{T}:=1_{T} \otimes \gamma: T \otimes_{\mathcal{A}\left(S_{1}\right)} V(\bigcirc)\right) \rightarrow T \otimes_{\mathcal{A}\left(S_{2}\right)} V(\Omega 0)\right) \\
& \left.\delta_{T}:=1_{T} \otimes \delta: T \otimes_{\mathcal{A}\left(S_{1}\right)} V(\bigcirc)\right) \rightarrow T \otimes_{\mathcal{A}\left(S_{2}\right)} V((30)) .
\end{aligned}
$$

From now on, we will simplify our notation even further, and denote the maps $\beta_{T}, \gamma_{T}$, $\delta_{T}$ in the following way:

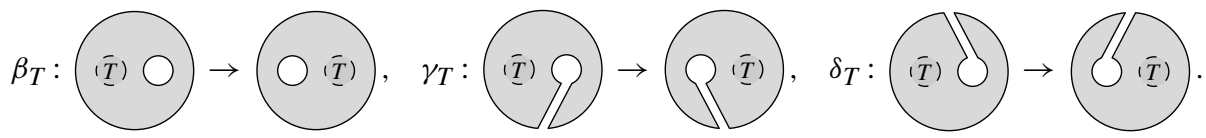

At this point, it becomes convenient to name some of the submanifolds of $\partial E$ and of $\partial F$ :

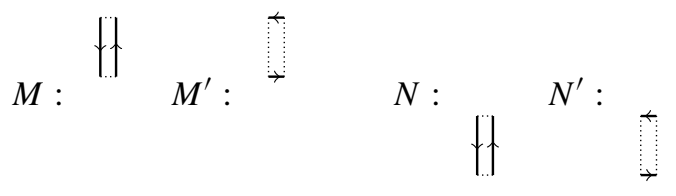

The maps $\beta_{T}, \gamma_{T}, \delta_{T}$, then satisfy the relations

$$
\gamma_{T} \otimes_{\mathcal{A}(N)} V(F)=\delta_{T} \otimes_{\mathcal{A}(M)} V(E)=\beta_{T} .
$$

Applying the functor $-\bigotimes_{\mathcal{A}\left(M^{\prime}\right) \bar{\otimes} \mathcal{A}\left(N^{\prime}\right)}(\overline{V(E)} \otimes \overline{V(F)})$ to the above equality, we get an equation in the space of maps from

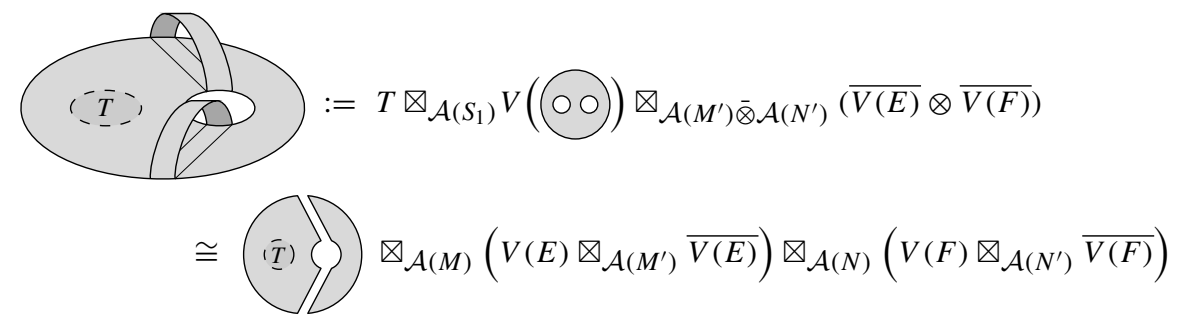

to

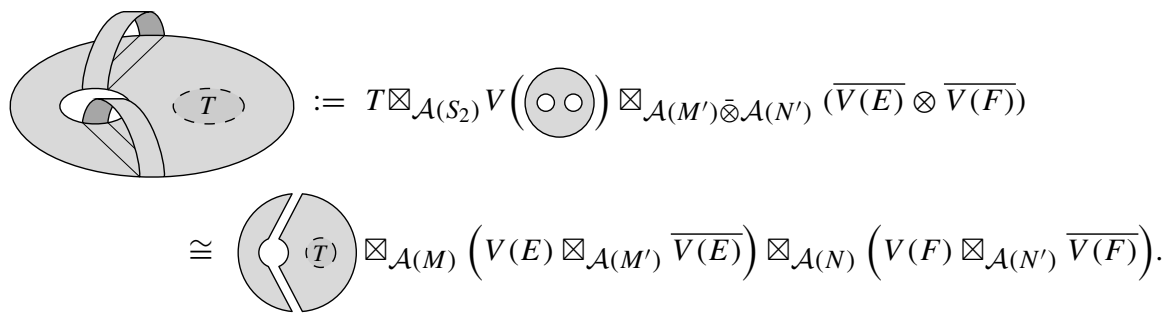


More specifically, we learn that the two maps

$$
\begin{aligned}
& \Gamma:=\left(\gamma_{T} \otimes_{\mathcal{A}(N)} V(F)\right) \otimes_{\mathcal{A}\left(M^{\prime}\right) \bar{\otimes} \mathcal{A}\left(N^{\prime}\right)} \overline{V(E)} \otimes \overline{V(F)} \\
&=\left(\gamma_{T} \otimes_{\mathcal{A}\left(M^{\prime}\right)} \overline{V(E)}\right) \otimes_{\mathcal{A}(N)}\left(V(F) \bigotimes_{\mathcal{A}\left(N^{\prime}\right)} \overline{V(F)}\right)
\end{aligned}
$$

and

$$
\begin{aligned}
\Delta:=( & \left.\delta_{T} \bigotimes_{\mathcal{A}(M)} V(E)\right) \bigotimes_{\mathcal{A}\left(M^{\prime}\right) \bar{\otimes} \mathcal{A}\left(N^{\prime}\right)} \overline{V(E)} \otimes \overline{V(F)} \\
= & \left(\delta_{T} \bigotimes_{\mathcal{A}\left(N^{\prime}\right)} \overline{V(F)}\right) \bigotimes_{\mathcal{A}(N)}\left(V(E) \bigotimes_{\mathcal{A}\left(M^{\prime}\right)} \overline{V(E)}\right)
\end{aligned}
$$

are both equal to

$$
B:=\beta_{T} \otimes_{\mathcal{A}\left(M^{\prime}\right) \bar{\otimes} \mathcal{A}\left(N^{\prime}\right)} \overline{V(E)} \otimes \overline{V(F)} .
$$

Summarizing, we have three equal maps (actually isomorphisms)

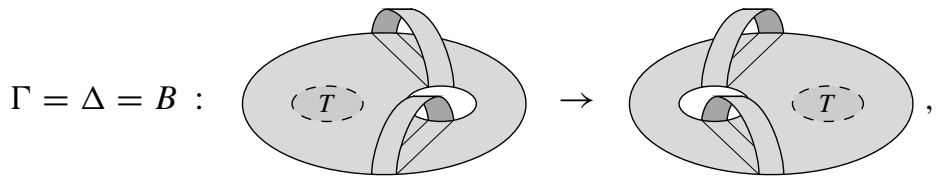

equivariant for $\mathcal{A}(\Omega)$ ) (where the actions of that algebra come from appropriate diffeomorphisms with the boundaries of the two 2-manifolds in (3.12)).

Since $\mathcal{A}(M)_{(E)} V\left(M^{\prime}\right)$ and $\mathcal{A}(N)_{V(F)} \mathcal{A}\left(N^{\prime}\right)$ are dualizable bimodules, we have inclusions $L^{2} \mathcal{A}(M) \subset V(E) \bigotimes_{\mathcal{A}\left(M^{\prime}\right)} \overline{V(E)}$ and $L^{2} \mathcal{A}(N) \subset V(F) \bigotimes_{\mathcal{A}\left(N^{\prime}\right)} \overline{V(F)}$. It follows that there is a diagram of inclusions

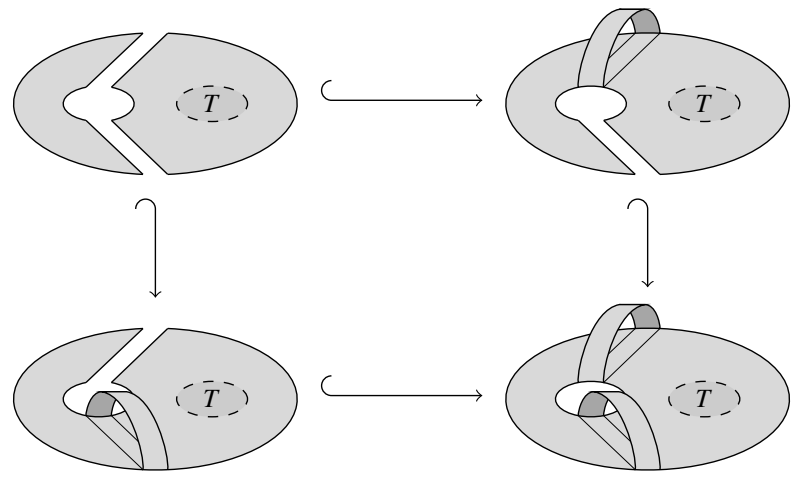

and that

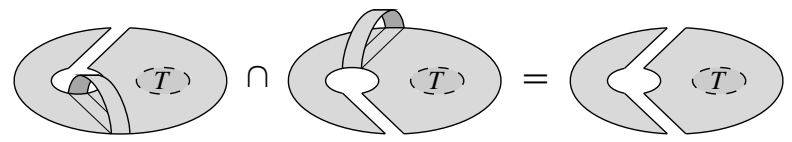

Note that the inclusions (3.13) are all compatible with the actions of $\mathcal{A}(\Omega)$ ) (where again the actions come from appropriate diffeomorphisms with the boundaries of the above 2-manifolds). 
Let us now look at the image of $\left(\bar{T}_{-}\right.$, Since $B=\Gamma$ is the result of applying $-\bigotimes_{\mathcal{A}(N)}\left(V(F) \bigotimes_{\mathcal{A}\left(N^{\prime}\right)} \overline{V(F)}\right)$ to some map, it follows that

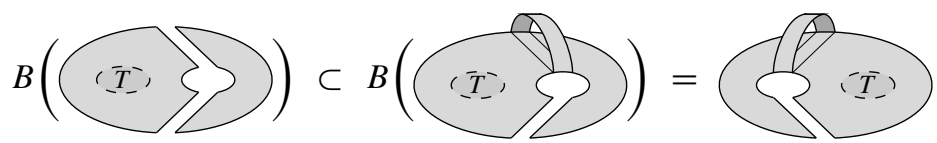

Similarly, since $B=\Delta$ is the result of applying $-\bigotimes_{\mathcal{A}(M)}\left(V(E) \bigotimes_{\mathcal{A}\left(M^{\prime}\right)} \overline{V(E)}\right)$ to some map, it follows that

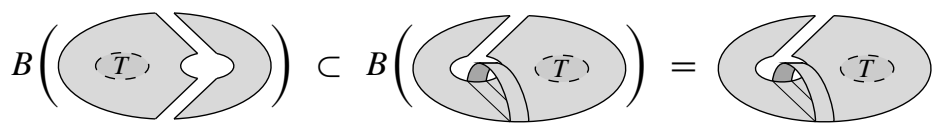

Combining (3.15), (3.16) and (3.14), we learn that the map $B$ sends $(T)\}$ to $\{(T)$. Recall that $B$ intertwines the actions of $\mathcal{A}\left(\Omega(\Omega)\right.$. The representations $T \otimes H_{0}$ and $H_{0} \otimes T$ of that algebra are irreducible; $B$ therefore induces an isomorphism $T \otimes H_{0} \cong H_{0} \otimes T$. It follows that $T \cong H_{0}$ (and $H_{0} \cong T$ ).

Open Access This article is distributed under the terms of the Creative Commons Attribution 4.0 International License (http://creativecommons.org/licenses/by/4.0/), which permits unrestricted use, distribution, and reproduction in any medium, provided you give appropriate credit to the original author(s) and the source, provide a link to the Creative Commons license, and indicate if changes were made.

\section{Appendix A. Cyclic Fusion and Fusion Along Graphs}

Let $\Gamma$ be a finite oriented graph ${ }^{9}$ for which the two vertices of each edge are distinct. Suppose that we are given, for every edge $e \in \Gamma_{1}$ a von Neumann algebra $A_{e}$, and for every vertex $v \in \Gamma_{0}$ a Hilbert space $H_{v}$. Assume furthermore that each $H_{v}$ is equipped with a left action of $A_{e}$ for every incoming edge $e$, and a right action of $A_{e}$ for every outgoing edge. Moreover, the actions on $H_{v}$ are required to be split, meaning that they extend to an action of the spatial tensor product

$$
\left(\bigotimes_{\substack{\text { incoming } \\ \text { edges } e}}^{\bar{\bigotimes}} A_{e}\right) \bar{\otimes}\left(\bigotimes_{\substack{\text { outgoing } \\ \text { edges } e}}^{\bar{\bigotimes}} A_{e}^{o p}\right)
$$

The graph fusion, denoted $\bigotimes_{\left\{A_{e}\right\}}\left\{H_{v}\right\}_{e, v \in \Gamma}$ (or simply $\bigotimes\left\{H_{v}\right\}_{v \in \Gamma}$, when the algebras $A_{e}$ are obvious from the context), is the Connes fusion of all the Hilbert spaces $H_{v}$ along all the algebras $A_{e}$.

Let us write $e \triangleright v$ to denote that $e$ in an incoming edge for $v$, and $e \triangleleft v$ to denote that $e$ is an outgoing edge for $v$.

\footnotetext{
${ }^{9}$ Here, by a graph we mean combinatorial graph, unlike the prevalent usage of the word graph in the body of the article, where it refers to a particular kind of topological space.
} 
Definition A.1. Let $\Gamma,\left\{H_{v}\right\}_{v \in \Gamma_{0}},\left\{A_{e}\right\}_{e \in \Gamma_{1}}$ be as above. The graph fusion

$$
\bigotimes_{\left\{A_{e}\right\}}\left\{H_{v}\right\}_{e, v \in \Gamma}
$$

of the Hilbert spaces $H_{v}$ over the algebras $A_{e}$ is the completion of the vector space

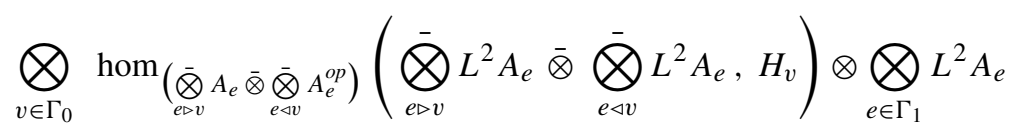

with respect to the pre-inner product

$$
\left\langle\bigotimes_{v \in \Gamma_{0}} \varphi_{v} \otimes \bigotimes_{e \in \Gamma_{1}} \xi_{e}, \bigotimes_{v \in \Gamma_{0}} \psi_{v} \otimes \bigotimes_{e \in \Gamma_{1}} \eta_{e}\right\rangle:=\left\langle\left(\prod_{v \in \Gamma_{0}}\left(\psi_{v}^{*} \varphi_{v}\right)\right)\left(\bigotimes_{e \in \Gamma_{1}} \xi_{e}\right), \bigotimes_{e \in \Gamma_{1}} \eta_{e}\right\rangle_{\bigotimes_{e \in \Gamma_{1}} L^{2} A_{e}}
$$

Note that it is important for $\Gamma$ not to have any loops since, otherwise, the action of the element $\psi_{v}^{*} \varphi_{v} \in \bar{\bigotimes}_{e \triangleright v} A_{e} \bar{\otimes} \bar{\bigotimes}_{e \triangleleft v} A_{e}^{o p}$ on the Hilbert space $\bigotimes_{e \in \Gamma_{1}} L^{2} A_{e}$ is ill-defined, as the left and right actions of an algebra $A$ on its $L^{2}$-space are typically not split. This is essentially the same issue as in Warning A.6 below.

Given a subset $S \subset \Gamma_{1}$ of edges, consider the graph $\tilde{\Gamma}$ obtained by reversing the orientations of all the edges in $S$. Given algebras $A_{e}$ indexed by $e \in \Gamma_{1}$, we can form new ones $\tilde{A}_{e}$ by letting $\tilde{A}_{e}:=A_{e}^{o p}$ if $e \in S$ and $\tilde{A}_{e}:=A_{e}$ if $e \notin S$. It is then immediate from the definition that

$$
\bigotimes_{\left\{\tilde{A}_{e}\right\}}\left\{H_{v}\right\}_{e, v \in \tilde{\Gamma}}=\bigotimes_{\left\{A_{e}\right\}}\left\{H_{v}\right\}_{e, v \in \Gamma}
$$

Now consider an equivalence relation $\mathcal{R}$ on the set $\Gamma_{0}$ of vertices of $\Gamma$. The quotient graph $\Gamma / \mathcal{R}$ is defined as

$$
(\Gamma / \mathcal{R})_{0}:=\Gamma_{0} / \mathcal{R} \quad \text { and } \quad(\Gamma / \mathcal{R})_{1}:=\left\{e \in \Gamma_{1} \mid \begin{array}{c}
e \text { connects vertices from } \\
\text { different equivalence classes }
\end{array}\right\} .
$$

For each equivalence class $V \in \Gamma_{0} / \mathcal{R}$, let $\Gamma_{V}$ denote the corresponding full subgraph of $\Gamma$. Given algebras $\left\{A_{e}\right\}_{e \in \Gamma_{1}}$ and Hilbert spaces $\left\{H_{v}\right\}_{v \in \Gamma_{0}}$, the graph fusion satisfies the following version of associativity:

$$
\bigotimes_{\left\{A_{E}\right\}}\left\{\bigotimes_{\left\{A_{e}\right\}}\left\{H_{v}\right\}_{e, v \in \Gamma_{V}}\right\}_{E, V \in \Gamma / \mathcal{R}}=\bigotimes_{\left\{A_{e}\right\}}\left\{H_{v}\right\}_{v \in \Gamma}
$$

The following result is the simplest special case of the above equation:

Lemma A.4. Let $A_{1}$ and $A_{2}$ be von Neuman algebras. Let $K_{i}$ be left $A_{i}$-modules, and let $H$ be a right $A_{1} \bar{\otimes} A_{2}$-module. Then there is a canonical isomorphism

$$
H \otimes_{A_{1} \otimes A_{2}}\left(K_{1} \otimes K_{2}\right) \cong K_{1} \otimes_{A_{1}^{o p}} H \otimes_{A_{2}} K_{2} .
$$


There is an important special case of graph fusion, called cyclic fusion, which is the case when the graph $\Gamma$ is a cycle. Let $n \geq 2$ be some number. For each $i \in\{1, \ldots, n\}$, let $A_{i}$ be a von Neumann algebra, and let $H_{i}$ be an $A_{i} \bar{\otimes} A_{i+1}^{o p}$-module (cyclic numbering). Then for each $i, j \in\{1, \ldots, n\}$, we can form the fusion of $H_{i} \bigotimes_{A_{i+1}} \ldots \bigotimes_{A_{j-1}} H_{j-1}$ (cyclic numbering) with $H_{j} \bigotimes_{A_{j+1}} \ldots \bigotimes_{A_{i-1}} H_{i-1}$ over the algebra $A_{i}^{o p} \bar{\otimes} A_{j}$. Under the above conditions, the Hilbert space

$$
\left(H_{i} \otimes_{A_{i+1}} \ldots \otimes_{A_{j-1}} H_{j-1}\right) \underset{A_{i}^{o p} \bar{\otimes} A_{j}}{\otimes}\left(H_{j} \otimes_{A_{j+1}} \ldots \bigotimes_{A_{i-1}} H_{i-1}\right)
$$

is isomorphic to the graph fusion of the $H_{i}$ 's and therefore independent, up to canonical unitary isomorphism, of the choices of $i$ and $j$. We call the above Hilbert space the cyclic fusion of the $H_{i}$ 's, and denote it by

$$
, H_{1} \bigotimes_{A_{2}} \cdots \bigotimes_{A_{n}} H_{n} \bigotimes_{A_{1}-}
$$

Warning A.6. For the space (A.5) to be well defined, it is crucial to have $n \geq 2$. In other words, given an $A \bar{\otimes} A^{o p}$ module $H$, the expression , $H \bigotimes_{A}$; is ill-defined. This can be seen by analyzing the example $H:={ }_{A} L^{2} A \otimes_{\mathbb{C}} L^{2} A_{A}$. In that case, one might expect to find,$H \nabla_{A} ;=L^{2} A$. However, there is in general no meaningful way of letting End $_{A \bar{\otimes} A^{o p}(\bar{H})}$ act on that Hilbert space.

There is one exception to this warning: if $A$ is a direct sum of type $I$ factors, then $H \bigotimes_{A}$; , still makes sense. It can be defined as $H \bigotimes_{A \bar{\otimes}} A^{o p} L^{2}(A)$, which is now meaningful because $A \bar{\otimes} A^{o p}$ does act on $L^{2}(A)$.

\section{Appendix B. The Hilbert Space Associated to an Annulus}

Let $S$ be circle, and let $\Sigma=S \times[0,1]$. We have seen in (1.6) how to associate, non-canonically, a Hilbert space $H_{\Sigma} \in \operatorname{Sect}_{S \sqcup \bar{S}}(\mathcal{A})$ to this annulus. Later, in (1.18), we learned that there is a non-canonical unitary isomorphism of $S \sqcup \bar{S}$-sectors

$$
H_{\Sigma} \cong \bigoplus_{\lambda \in \Delta} H_{\lambda}(S) \otimes H_{\bar{\lambda}}(\bar{S})
$$

Note that, by (1.21), this can be reinterpreted as an isomorphism

$$
H_{\Sigma} \cong L^{2} \mathcal{A}(S)
$$

The goal of this section is to redefine $H_{\Sigma}$ in such a way that it becomes well defined up to canonical unitary isomorphism, and to upgrade (B.1) to a canonical unitary isomorphism.

Let $\mathcal{I}=\left\{I_{1}, \ldots, I_{n}\right\}$ be a $c$-cover of $S$. The intervals $I_{j}$ are arranged so that each intersection $p_{i}:=I_{i-1} \cap I_{i}$ (cyclic numbering) is a single point (or two points if $n=2$ ). By definition, $H_{\Sigma}$ is then the fusion of all the vacuum sectors $H_{i}:=H_{0}\left(\partial I_{i} \times[0,1]\right)$ along all the algebras $A_{i}:=\mathcal{A}\left(\left\{p_{i}\right\} \times[0,1]\right)$.

The first reason $H_{\Sigma}$ isn't canonically defined is that the Hilbert spaces $H_{i}$ themselves are only well defined up to non-canonical unitary isomorphism. One can fix that issue by being more specific: from now on, $H_{i}$ will denote the vacuum sector of $\mathcal{A}$ associated to 
the circle $S_{i}:=\partial I_{i} \times[0,1]$, its upper half $S_{i}^{\top}:=S_{i} \cap\left(I_{i} \times[1 / 2,1]\right)$, and the involution $j: S_{i} \rightarrow S_{i}, j(x, t)=(x, 1-t)$, as described near the beginning of Sect. 1 .

Our next task is to show that $H_{\Sigma}$ is independent of the $c$-cover $\mathcal{I}$. In order to do so, it is useful to introduce a notation that stresses the dependence:

$$
H_{\Sigma}^{(\mathcal{I})}:=, H_{1} \bigotimes_{A_{2}} \cdots \bigotimes_{A_{n}} H_{n} \bigotimes_{A_{1}-}
$$

The dashed line denotes the operation of cyclic fusion, described in Appendix A.

Given two $c$-covers $\mathcal{I}$ and $\mathcal{J}$ of $S$, we need to construct a unitary isomorphism between $H_{\Sigma}^{(\mathcal{I})}$ and $H_{\Sigma}^{(\mathcal{J})}$. As in the proof of Lemma 1.9, note that one can go from any cover to any other one by subdividing and recombining intervals. It is therefore enough to treat the case when $\mathcal{I}=\left\{I_{1}, I_{2}, \ldots, I_{n}\right\}$ and $\mathcal{J}=\left\{I_{1}^{\prime}, I_{1}^{\prime \prime}, I_{2}, \ldots, I_{n}\right\}$ with $I_{1}^{\prime} \cup I_{1}^{\prime \prime}=I_{1}$. Our first goal is therefore to upgrade the non-canonical isomorphism (1.10) to a canonical unitary isomorphism. This will be the content of Lemma B.4.

Let $I$ be an interval and let $\left\{I_{1}, I_{2}\right\}$ be a $c$-cover of $I$. Let $\{p, q, r\}=\partial I_{1} \cup \partial I_{2}$, with $\partial I_{1}=\{p, q\}, \partial I_{2}=\{q, r\}, \partial I=\{p, r\}$, and let $S_{1}=\partial\left(I_{1} \times[0,1]\right), S_{2}=\partial\left(I_{2} \times[0,1]\right)$, $S_{3}=\partial(I \times[0,1])$. Write $j$ for the involution $j(x, t)=(x, 1-t)$ on $I \times[0,1]$. Finally, let $S_{a}^{\top}=S_{a} \cap\left(I \times\left[\frac{1}{2}, 1\right]\right)$ for $a \in\{1,2,3\}$, and let $K=q \times[0,1]$. We orient $S_{1}, S_{2}$, $S_{3}, S_{1}^{\top}, S_{2}^{\top}, S_{3}^{\top}, K$ as follows:
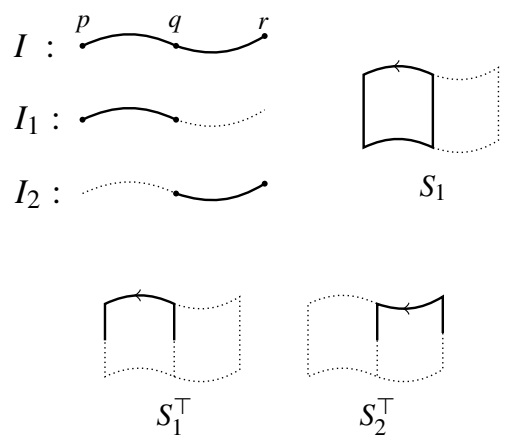

$S_{1}$
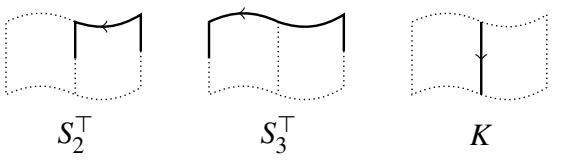

Lemma B.4. With $S_{1}, S_{2}, S_{3}, j, S_{1}^{\top}, S_{2}^{\top}, S_{3}^{\top}, K$ as above, let us define

$$
H_{a}:=L^{2} \mathcal{A}\left(S_{a}^{\top}\right) \in \operatorname{Sect}_{S_{a}}(\mathcal{A}), \quad a \in\{1,2,3\}
$$

to be the vacuum sector of $\mathcal{A}$ associated to $S_{a}, S_{a}^{\top}$, and $j$. Then there is a canonical unitary isomorphism of $S_{3}$-sectors

$$
u: H_{1} \otimes_{K} H_{2} \stackrel{\cong}{\longrightarrow} H_{3} .
$$

Proof. Let $S^{1}$ be the standard circle, let

$S_{\text {† }}^{1}:=\left\{z \in S^{1} \mid \Im \operatorname{m}(z) \geq 0\right\}, S_{\vdash}^{1}:=\left\{z \in S^{1} \mid \Re \mathrm{Re}(z) \leq 0\right\}, S_{\dashv}^{1}:=\left\{z \in S^{1} \mid \Re \mathrm{e}(z) \geq 0\right\}$,

and let $\psi_{\vdash}:[0,1] \rightarrow S_{\vdash}^{1}$ and $\psi_{\dashv}:[0,1] \rightarrow S_{\dashv}^{1}$ be the diffeomorphisms given by $\psi_{\vdash}(t)=-\sin (\pi t)-i \cos (\pi t)$ and $\psi_{\dashv}(t)=\sin (\pi t)-i \cos (\pi t)$. 
Let $p, q, r \in I$ be as above, and let us pick diffeomorphisms $f_{1}: S_{1} \rightarrow S^{1}$, $f_{2}: S_{2} \rightarrow S^{1}$ so that

$$
\begin{aligned}
\exists \varepsilon>0: \forall t \in\left[\frac{1}{2}-\varepsilon, \frac{1}{2}+\varepsilon\right]: & f_{1}(p, t)=\psi_{\vdash}(t), \quad f_{2}(r, t)=\psi_{\dashv}(t), \\
\forall t \in[0,1]: & f_{1}(q, t)=\psi_{\dashv}(t), \quad f_{2}(q, t)=\psi_{\vdash}(t),
\end{aligned}
$$

and $f_{a}(j(x))=\overline{f_{a}(x)}$. Finally, let $f_{3}:=\left.\left.f_{1}\right|_{S_{1} \cap S_{3}} \cup f_{2}\right|_{S_{2} \cap S_{3}}: S_{3} \rightarrow S^{1}$.

Recall from [8, Thm. 2.13] that there is an $\mathcal{A}$-sector $H_{0}=H_{0}\left(S^{1}, \mathcal{A}\right)$ that is canonically associated to the standard circle $S^{1}$. It is equipped, among others, with isomorphisms of $S^{1}$-sectors $v_{\top}: H_{0} \rightarrow L^{2}\left(\mathcal{A}\left(S_{\top}^{1}\right)\right)$ and $v_{\vdash}: H_{0} \rightarrow L^{2}\left(\mathcal{A}\left(S_{\vdash}^{1}\right)\right)$. The isomorphism (B.5) is then the composite

$$
\begin{aligned}
u: H_{1} \bigotimes_{K} H_{2} & =L^{2} \mathcal{A}\left(S_{1}^{\top}\right) \bigotimes_{K} L^{2} \mathcal{A}\left(S_{2}^{\top}\right) \stackrel{L^{2} \mathcal{A}\left(f_{1}\right) \otimes L^{2} \mathcal{A}\left(f_{2}\right)}{\longrightarrow} \\
& \rightarrow L^{2} \mathcal{A}\left(S_{\top}^{1}\right) \bigotimes_{K} L^{2} \mathcal{A}\left(S_{\top}^{1}\right) \stackrel{1 \otimes v_{\top}^{-1}}{\longrightarrow} L^{2} \mathcal{A}\left(S_{\top}^{1}\right) \bigotimes_{K} H_{0} \\
& =L^{2} \mathcal{A}\left(S_{\top}^{1}\right) \bigotimes_{\mathcal{A}\left(S_{\vdash}^{1}\right)} H_{0} \stackrel{1 \bigotimes v_{\vdash}}{\longrightarrow} L^{2} \mathcal{A}\left(S_{\top}^{1}\right) \bigotimes_{\mathcal{A}\left(S_{\vdash}^{1}\right)} L^{2} \mathcal{A}\left(S_{\vdash}^{1}\right) \\
& \cong L^{2} \mathcal{A}\left(S_{\top}^{1}\right) \stackrel{L^{2} \mathcal{A}\left(f_{3}\right)^{-1}}{\longrightarrow} L^{2} \mathcal{A}\left(S_{3}^{\top}\right)=H_{3} .
\end{aligned}
$$

We still need to show that $u$ is independent of the choices made. For that, we pick new diffeomorphisms $\tilde{f}_{a}: S_{a} \rightarrow S^{1}$ with the same properties as the maps $f_{a}$, and define

$$
\tilde{u}:=\left(L^{2} \mathcal{A}\left(\tilde{f}_{3}\right)^{-1}\right) \circ\left(1 \otimes v_{\vdash} v_{\top}^{-1}\right) \circ\left(L^{2} \mathcal{A}\left(\tilde{f}_{1}\right) \otimes L^{2} \mathcal{A}\left(\tilde{f}_{2}\right)\right)
$$

as in (B.6). Let $g_{a}:=\left.\left(\tilde{f}_{a} \circ f_{a}^{-1}\right)\right|_{S_{\top}^{1}}$, and note that $g_{3}=g_{1} \circ g_{2}$. By the inner covariance axiom, there exist unitaries $w_{a} \in \mathcal{A}\left(S_{\top}^{1}\right)$ so that $\mathcal{A}\left(g_{a}\right)=\operatorname{Ad}\left(w_{a}\right)$. Moreover, we can chose $w_{3}=w_{1} w_{2}$. Let $j_{0}: S^{1} \rightarrow S^{1}$ denote complex conjugation, and let $W_{a}$ be the operator given by multiplication by $w_{1}$ followed by multiplication by $\mathcal{A}\left(j_{0}\right)\left(w_{1}^{*}\right)$. To show that $\tilde{u}=u$, we need to argue that following diagrams are commutative:
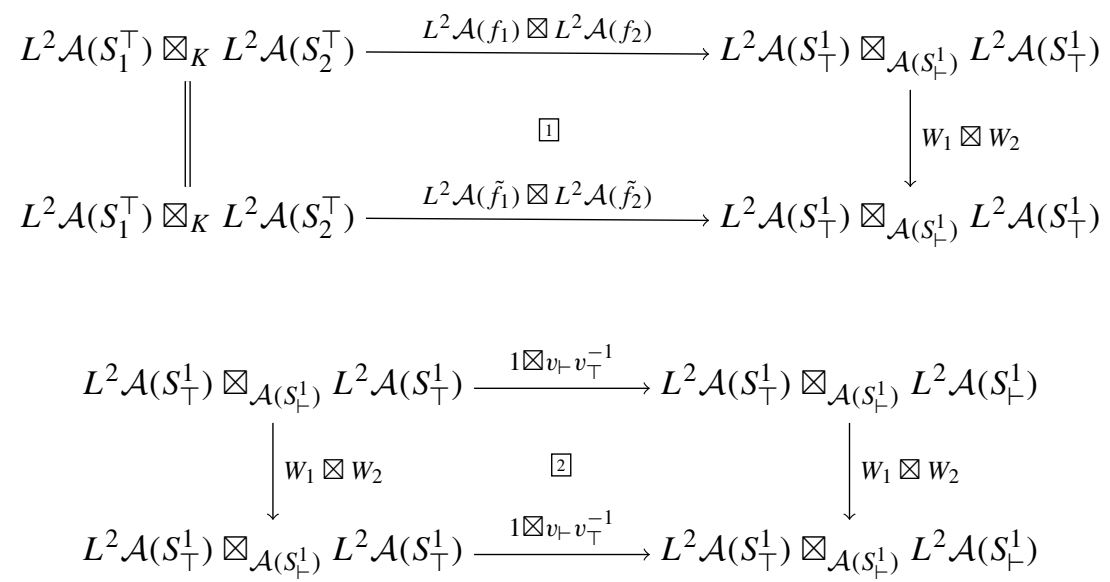


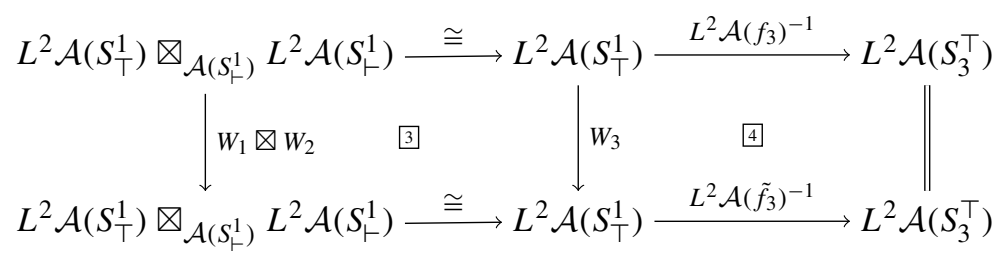

The commutativity of $\square$ and 3 is obvious. Finally, the commutativity of $\square$ and 4 follows from the following general fact: given a von Neumann algebra $A$, a unitary $u \in \mathrm{U}(A)$ and a vector $\xi \in L^{2}(A)$, one always has $L^{2}(\operatorname{Ad}(u))(\xi)=u \xi u^{*}$.

Note that despite its apparent asymmetry, the definition (B.6) is left-right symmetric. Indeed, we could have used

$$
L^{2} \mathcal{A}\left(S_{\top}^{1}\right) \otimes_{\mathcal{A}\left(S_{\vdash}^{1}\right)} L^{2} \mathcal{A}\left(S_{\top}^{1}\right) \stackrel{v_{\vdash} v_{\top}^{-1} \boxplus 1}{\longrightarrow} L^{2} \mathcal{A}\left(S_{\vdash}^{1}\right) \otimes_{\mathcal{A}\left(S_{\vdash}^{1}\right)} L^{2} \mathcal{A}\left(S_{\top}^{1}\right) \cong L^{2} \mathcal{A}\left(S_{\top}^{1}\right)
$$

instead of

$$
L^{2} \mathcal{A}\left(S_{\top}^{1}\right) \bigotimes_{\mathcal{A}\left(S_{\vdash}^{1}\right)} L^{2} \mathcal{A}\left(S_{\top}^{1}\right) \stackrel{1 \bigotimes v_{\vdash} v_{\top}^{-1}}{\longrightarrow} L^{2} \mathcal{A}\left(S_{\top}^{1}\right) \bigotimes_{\mathcal{A}\left(S_{\vdash}^{1}\right)} L^{2} \mathcal{A}\left(S_{\vdash}^{1}\right) \cong L^{2} \mathcal{A}\left(S_{\top}^{1}\right)
$$

in the middle of (B.6) as both are equal to

$$
\begin{gathered}
L^{2} \mathcal{A}\left(S_{\top}^{1}\right) \otimes_{\mathcal{A}\left(S_{\vdash}^{1}\right)} L^{2} \mathcal{A}\left(S_{\top}^{1}\right) \stackrel{v_{\vdash} v_{\top}^{-1} \nabla_{\vdash} v_{\top}^{-1}}{\longrightarrow} L^{2} \mathcal{A}\left(S_{\vdash}^{1}\right) \otimes_{\mathcal{A}\left(S_{\vdash}^{1}\right)} L^{2} \mathcal{A}\left(S_{\vdash}^{1}\right) \cong \\
\cong L^{2} \mathcal{A}\left(S_{\vdash}^{1}\right) \stackrel{v_{\uparrow} v_{\vdash}^{-1}}{\longrightarrow} L^{2} \mathcal{A}\left(S_{\top}^{1}\right) .
\end{gathered}
$$

Let $S_{1}, S_{2}, S_{3}, H_{1}, H_{2}, H_{3}$ be as in Lemma B.4. Letting $J_{a}$ denote the modular conjugation on $H_{a}=L^{2}\left(\mathcal{A}\left(S_{a}^{\top}\right)\right), a \in\{1,2,3\}$, we expect the isomorphism (B.5) to satisfy

$$
u \circ\left(J_{1} \bigotimes_{\alpha} J_{2}\right)=J_{3} \circ u,
$$

where $\alpha: \mathcal{A}(K) \rightarrow \mathcal{A}(K)$ is the anti-linear homomorphism given by $\alpha(x)=\mathcal{A}(j)\left(x^{*}\right)$. We only know how to prove the above equation up to sign:

Lemma B.7. Let $u, J_{a}$, and $\alpha$ be as above. Then we have

$$
u \circ\left(J_{1} \bigotimes_{\alpha} J_{2}\right)= \pm J_{3} \circ u .
$$

Proof. Recall from [8, Thm. 2.13] that $\varphi \mapsto H_{0}(\varphi)$ is a representation of the group of Möbius transformations of $S^{1}$ on the vacuum sector $H_{0}=H_{0}\left(S^{1}\right)$. The operator $H_{0}(\varphi)$ is unitary for $\varphi$ orientation preserving, and antiunitary for $\varphi$ orientation reversing. Let $j_{0}: S^{1} \rightarrow S^{1}$ denote complex conjugation, and let $\beta: \mathcal{A}\left(S_{\vdash}^{1}\right) \rightarrow \mathcal{A}\left(S_{\vdash}^{1}\right)$ be given by $\beta(x)=\mathcal{A}\left(j_{0}\right)\left(x^{*}\right)$. Recall the definition (B.6) of the isomorphism $u$. In order to prove 
(B.8), it is enough to show that the following squares labelled 1, 2, 3, 4 commute up to sign
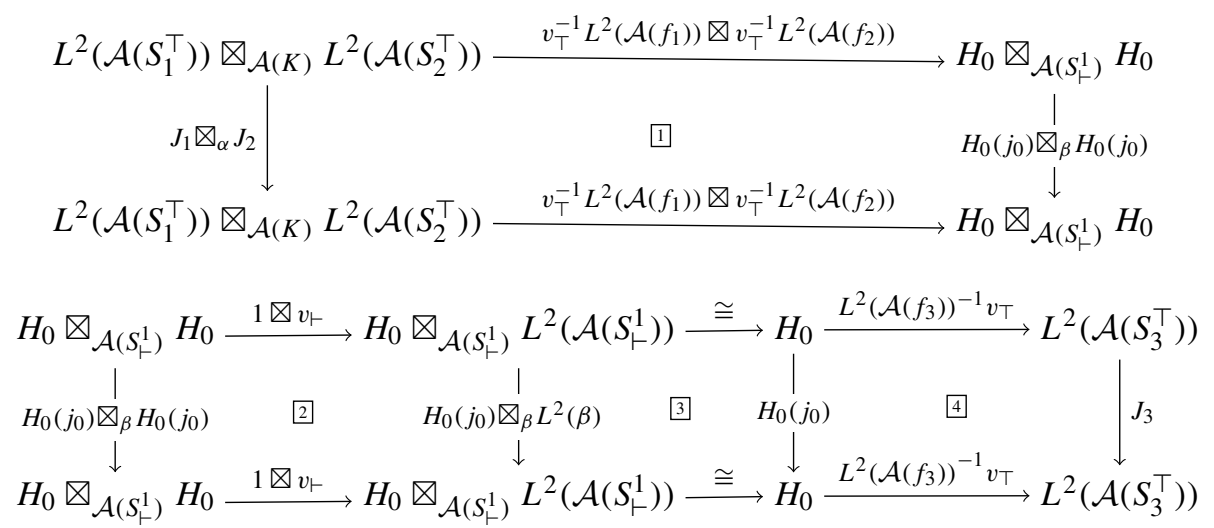

where $f_{1}, f_{2}, f_{3}$ are as in the proof of Lemma B.4. The squares 1 and 4 commute by [8, Thm. 2.13 (iv)], and 3 is easily seen to be commutative. So the only square that remains is 2 , which is equivalent to

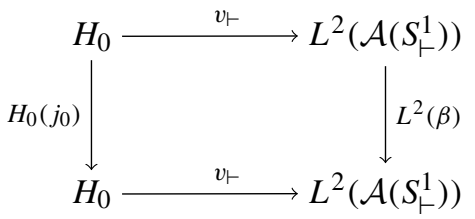

Note that by [8, Thm. 2.13 (ii)] (see also [8, Prop. 2.10]) and [8, Lem. 2.9] respectively, both $H_{0}\left(j_{0}\right)$ and $L^{2}(\beta)=L^{2}\left(\mathcal{A}\left(j_{0}\right)\right) \circ J$ implement $j_{0}$ ([8, Def. 2.1]). Since both of them are antilinear involutions that implement $j_{0}$, by Schur's lemma, they are equal up to sign.

The isomorphism $u: H_{1} \bigotimes_{K} H_{2} \cong H_{3}$ from (B.5) satisfies a certain version of associativity, which we now describe. Let $\left\{I_{1}, I_{2}, I_{3}\right\}$ be a $c$-cover of $I$. We call the boundary points $p, q, r, s$ and assume that they are arranged as follows:

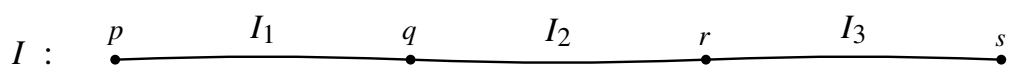

Consider the circles

$$
\begin{aligned}
S_{1}:=\partial\left(I_{1} \times[0,1]\right), & S_{2}:=\partial\left(I_{2} \times[0,1]\right), \quad S_{3}:=\partial\left(I_{3} \times[0,1]\right), \\
S_{12}:=\partial\left(\left(I_{1} \cup I_{2}\right) \times[0,1]\right), & S_{23}:=\partial\left(\left(I_{2} \cup I_{3}\right) \times[0,1]\right), \quad S_{123}:=\partial(I \times[0,1]),
\end{aligned}
$$

and let

$$
\begin{array}{cl}
H_{1}:=H_{0}\left(S_{1}\right), \quad H_{2}:=H_{0}\left(S_{2}\right), \quad H_{3}:=H_{0}\left(S_{3}\right), \\
H_{12}:=H_{0}\left(S_{12}\right), \quad H_{23}:=H_{0}\left(S_{23}\right), \quad H_{123}:=H_{0}\left(S_{123}\right)
\end{array}
$$


be the vacuum sectors associated the upper halves and to the involutions $j:(x, t) \mapsto$ $(x, 1-t)$. Let also $K:=\{q\} \times[0,1]$ and $L:=\{r\} \times[0,1]$. We then have the following four instances of the isomorphism (B.5):

$$
\begin{array}{cl}
u_{12}: H_{1} \bigotimes_{K} H_{2} \cong H_{12}, & u_{23}: H_{2} \bigotimes_{L} H_{3} \cong H_{23}, \\
u_{12,3}: H_{12} \bigotimes_{L} H_{3} \cong H_{123}, & u_{1,23}: H_{1} \bigotimes_{K} H_{23} \cong H_{123} .
\end{array}
$$

Lemma B.10. The above maps fit into a commutative diagram

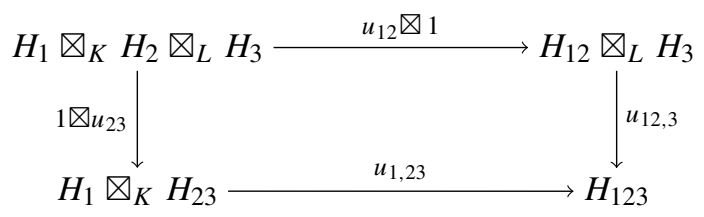

Proof. Letting $\psi_{\vdash}:[0,1] \rightarrow S_{\vdash}^{1}$ and $\psi_{\dashv}:[0,1] \rightarrow S_{\dashv}^{1}$ be as in the proof of Lemma B.4, and given diffeomorphisms $f: S_{1} \rightarrow S^{1}, g: S_{3} \rightarrow S^{1}$ subject to

$$
\begin{aligned}
& \exists \varepsilon>0: \forall t \in\left[\frac{1}{2}-\varepsilon, \frac{1}{2}+\varepsilon\right]: f(p, t)=\psi_{\vdash}(t), \quad g(s, t)=\psi_{\dashv}(t), \\
& \forall t \in[0,1]: \quad f(q, t)=\psi_{\dashv}(t), \quad g(r, t)=\psi_{\vdash}(t),
\end{aligned}
$$

one can write $u_{12,3} \circ\left(u_{12} \otimes 1\right)$ as

$$
\begin{array}{r}
H_{1} \otimes_{K} H_{2} \otimes_{L} H_{3} \stackrel{v_{\vdash} v_{\top}^{-1} L^{2} \mathcal{A}(f) \otimes 1 \otimes 1}{\longrightarrow} L^{2} \mathcal{A}\left(S_{\vdash}^{1}\right) \otimes_{\mathcal{A}\left(S_{\vdash}^{1}\right)} H_{2} \otimes_{L} H_{3} \\
\cong H_{2} \otimes_{L} H_{3} \stackrel{L^{2} \mathcal{A}\left(\left(f^{-1} \circ \psi_{\vdash}\right) \cup \mathrm{id}\right) \otimes 1}{\longrightarrow} H_{12} \otimes_{L} H_{3} \stackrel{1 \otimes v_{\vdash} v_{\top}^{-1} L^{2} \mathcal{A}(g)}{\longrightarrow} \\
\rightarrow H_{12} \otimes_{\mathcal{A}\left(S_{\vdash}^{1}\right)} L^{2} \mathcal{A}\left(S_{\vdash}^{1}\right) \cong H_{12} \stackrel{L^{2} \mathcal{A}\left(\mathrm{id} \cup\left(g^{-1} \circ \psi_{\dashv}\right)\right)}{\longrightarrow} H_{123}
\end{array}
$$

and $u_{1,23} \circ\left(1 \otimes u_{23}\right)$ as

$$
\begin{array}{r}
H_{1} \otimes_{K} H_{2} \otimes_{L} H_{3} \stackrel{1 \otimes 1 \otimes v_{\vdash} v_{\top}^{-1} L^{2} \mathcal{A}(g)}{\longrightarrow} H_{1} \otimes_{K} H_{2} \otimes_{\mathcal{A}\left(S_{\vdash}^{1}\right)} L^{2} \mathcal{A}\left(S_{\vdash}^{1}\right) \\
\cong H_{1} \otimes_{K} H_{2} \stackrel{1 \otimes L^{2} \mathcal{A}\left(\mathrm{id} \cup\left(g^{-1} \circ \psi_{-}\right)\right)}{\longrightarrow} H_{1} \otimes_{K} H_{23} \stackrel{v_{\vdash} v_{\top}^{-1} L^{2} \mathcal{A}(f) \otimes 1}{\longrightarrow} \\
\rightarrow L^{2} \mathcal{A}\left(S_{\vdash}^{1}\right) \otimes_{\mathcal{A}\left(S_{\vdash}^{1}\right)} H_{23} \cong H_{23} \stackrel{L^{2} \mathcal{A}\left(\left(f^{-1} \circ \psi_{\vdash}\right) \cup \mathrm{id}\right)}{\longrightarrow} H_{123} .
\end{array}
$$

Both composites are equal to

$$
\begin{gathered}
H_{1} \otimes_{K} H_{2} \bigotimes_{L} H_{3} \stackrel{v_{\vdash} v_{\top}^{-1} L^{2} \mathcal{A}(f) \otimes 1 \otimes v_{\vdash} v_{\top}^{-1} L^{2} \mathcal{A}(g)}{\longrightarrow} \\
L^{2} \mathcal{A}\left(S_{\vdash}^{1}\right) \bigotimes_{\mathcal{A}\left(S_{\vdash}^{1}\right)} H_{2} \otimes_{\mathcal{A}\left(S_{\vdash}^{1}\right)} L^{2} \mathcal{A}\left(S_{\vdash}^{1}\right) \cong H_{2} \stackrel{L^{2} \mathcal{A}\left(\left(f^{-1} \circ \psi_{\vdash}\right) \cup \mathrm{id} \cup\left(g^{-1} \circ \psi_{\dashv}\right)\right)}{\longrightarrow} H_{123}
\end{gathered}
$$

We can now prove that the Hilbert space associated to $\Sigma=S \times[0,1]$ is well defined up to unique unitary isomorphism. Given a $c$-cover $\mathcal{I}$ of $S$, let

$$
H_{\Sigma}^{(\mathcal{I})}:=, \ldots H_{1} \bigotimes_{A_{2}} \cdots \bigotimes_{A_{n}} H_{n} \bigotimes_{A_{1}-}, \in \operatorname{Sect}_{S \sqcup \bar{S}}(\mathcal{A})
$$

be the Hilbert space defined in (B.2). 
Proposition B.11. Given two c-covers $\mathcal{I}_{1}$ and $\mathcal{I}_{2}$ of a circle $S$, there is a canonical unitary isomorphism of $S \sqcup \bar{S}$-sectors

$$
u^{\left(\mathcal{I}_{1}, \mathcal{I}_{2}\right)}: H_{\Sigma}^{\left(\mathcal{I}_{1}\right)} \longrightarrow H_{\Sigma}^{\left(\mathcal{I}_{2}\right)}
$$

Moreover, given three c-covers $\mathcal{I}_{1}, \mathcal{I}_{2}, \mathcal{I}_{3}$ of $S$, the following diagram commutes:

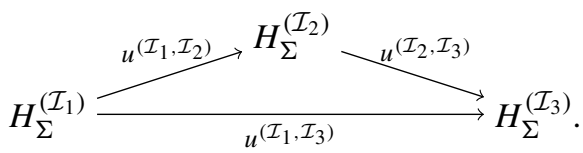

Proof. Let $\mathcal{I}_{1}$ and $\mathcal{I}_{2}$ be two $c$-covers of $S$. If $\mathcal{I}_{1}$ is a refinement of $\mathcal{I}_{2}$, then we may pick a sequence of $c$-covers $\mathcal{I}_{1}=\mathcal{J}_{1} \prec \mathcal{J}_{2} \prec \cdots \prec \mathcal{J}_{n}=\mathcal{I}_{2}$ such that each $\mathcal{J}_{n}$ is obtained from the next one $\mathcal{J}_{n+1}$ by subdividing some interval in two. Using (B.5) in (1.11) produces a canonical isomorphisms of $S \sqcup \bar{S}$-sectors $v_{i}: H_{\Sigma}^{\left(\mathcal{J}_{i}\right)} \rightarrow H_{\Sigma}^{\left(\mathcal{J}_{i+1}\right)}$. By Lemma B.10, the composite

$$
v^{\left(\mathcal{I}_{1}, \mathcal{I}_{2}\right)}:=v_{n-1} \circ \cdots \circ v_{2} \circ v_{1}: H_{\Sigma}^{\left(\mathcal{I}_{1}\right)} \longrightarrow H_{\Sigma}^{\left(\mathcal{I}_{2}\right)}
$$

is independent of the choice of intermediate $c$-covers and it is straightforward to check that the above isomorphisms satisfy $v^{\left(\mathcal{I}_{2}, \mathcal{I}_{3}\right)} \circ v^{\left(\mathcal{I}_{1}, \mathcal{I}_{2}\right)}=v^{\left(\mathcal{I}_{1}, \mathcal{I}_{3}\right)}$.

Now given two arbitrary $c$-covers $\mathcal{I}_{1}$ and $\mathcal{I}_{2}$, we proceed as in Lemma 1.9. Chose $c$-covers $\mathcal{J}, \mathcal{I}^{\prime}, \mathcal{J}^{\prime}$ so that $\mathcal{I}_{1} \succ \mathcal{J} \prec \mathcal{I}^{\prime} \succ \mathcal{J}^{\prime} \prec \mathcal{I}_{2}$ and set

$$
u^{\left(\mathcal{I}_{1}, \mathcal{I}_{2}\right)}:=v^{\left(\mathcal{J}^{\prime}, \mathcal{I}_{2}\right)}\left(v^{\left(\mathcal{J}^{\prime}, \mathcal{I}^{\prime}\right)}\right)^{-1} v^{\left(\mathcal{J}, \mathcal{I}^{\prime}\right)}\left(v^{\left(\mathcal{J}, \mathcal{I}_{1}\right)}\right)^{-1}: H_{\Sigma}^{\left(\mathcal{I}_{1}\right)} \longrightarrow H_{\Sigma}^{\left(\mathcal{I}_{2}\right)}
$$

It is then fairly easy to verify that $u^{\left(\mathcal{I}_{1}, \mathcal{I}_{2}\right)}$ is independent of the choice of intermediate $c$-covers $\mathcal{J}, \mathcal{I}^{\prime}, \mathcal{J}^{\prime}$, and that it satisfies $u^{\left(\mathcal{I}_{2}, \mathcal{I}_{3}\right)} \circ u^{\left(\mathcal{I}_{1}, \mathcal{I}_{2}\right)}=u^{\left(\mathcal{I}_{1}, \mathcal{I}_{3}\right)}$.

Given an interval $I$ with local coordinates around its endpoints, let us denote by $\partial(I \times[0,1])_{\top}:=\left(I \times\left[\frac{1}{2}, 1\right]\right) \cap \partial(I \times[0,1])$, the upper half of $\partial(I \times[0,1])$. As a corollary, we have the following result:

Theorem B.13. Let $S$ be a circle. Then the Hilbert space $H_{\Sigma} \in \operatorname{Sect}_{S \sqcup \bar{S}}(\mathcal{A})$ associated to the annulus $\Sigma=S \times[0,1]$ is well defined up to canonical unitary isomorphism.

For every c-cover $\mathcal{I}=\left\{I_{1}, \ldots, I_{n}\right\}$ (Definition 1.8) of the circle $S$, there is a canonical unitary isomorphism of $S \sqcup \bar{S}$-sectors

$$
u^{(\mathcal{I})}: H_{\Sigma} \longrightarrow H_{\Sigma}^{(\mathcal{I})}=,-H_{1} \bigotimes_{A_{2}} \cdots \bigotimes_{A_{n}} H_{n} \bigotimes_{A_{1}-} \text {; }
$$

where

$$
\begin{gathered}
H_{i}=L^{2} \mathcal{A}\left(\partial\left(I_{i} \times[0,1]\right) \top\right) \in \operatorname{Sect}_{\partial I_{i} \times[0,1]}(\mathcal{A}) \\
\text { and } \quad A_{i}=\mathcal{A}\left(\left(I_{i-1} \cap I_{i}\right) \times[0,1]\right)
\end{gathered}
$$

are as in (B.2).

Moreover, given two c-covers $\mathcal{I}_{1}, \mathcal{I}_{2}$, the composite $u^{\left(\mathcal{I}_{2}\right)} \circ\left(u^{\left(\mathcal{I}_{1}\right)}\right)^{-1}$ is the map $u^{\left(\mathcal{I}_{1}, \mathcal{I}_{2}\right)}$ from Proposition B.11. 
Proof. Let us define an element $\xi \in H_{\Sigma}$ to be a family of vectors $\left\{\xi^{(\mathcal{I})} \in H_{\Sigma}^{(\mathcal{I})}\right\}_{\mathcal{I}}$ indexed by all $c$-covers $\mathcal{I}$ of $S$, subject to the condition

$$
u^{\left(\mathcal{I}_{1}, \mathcal{I}_{2}\right)}\left(\xi^{\left(\mathcal{I}_{1}\right)}\right)=\xi^{\left(\mathcal{I}_{2}\right)} \quad \forall \mathcal{I}_{1}, \mathcal{I}_{2} .
$$

Addition and scalar multiplication are defined pointwise. The inner product is given by $\left\langle\left\{\xi^{(\mathcal{I})}\right\},\left\{\eta^{(\mathcal{I})}\right\}\right\rangle:=\left\langle\xi^{\left(\mathcal{I}_{0}\right)}, \eta^{\left(\mathcal{I}_{0}\right)}\right\rangle$ for any $c$-cover $\mathcal{I}_{0}$, and is well defined because the maps $u^{\left(\mathcal{I}_{1}, \mathcal{I}_{2}\right)}$ are unitary. Finally, the map $u^{(\mathcal{J})}$ sends a family $\left\{\xi^{(\mathcal{I})}\right\}_{\mathcal{I}}$ to its $\mathcal{J}$-th element $\xi^{(\mathcal{J})}$, and the inverse map sends $\eta \in H_{\Sigma}^{(\mathcal{J})}$ to the family $\left\{u^{(\mathcal{J}, \mathcal{I})}(\eta)\right\}_{\mathcal{I}}$. The latter sign satisfies (B.15) by (B.12).

As a consequence of Lemma B.7, the Hilbert space $H_{\Sigma}$ is equipped with an antilinear involution $J_{\Sigma}: H_{\Sigma} \rightarrow H_{\Sigma}$ given by

$$
\begin{aligned}
& J_{\Sigma}: H_{\Sigma} \stackrel{u^{(\mathcal{I})}}{\longrightarrow}, . H_{1} \bigotimes_{A_{2}} \ldots \bigotimes_{A_{n}} H_{n} \bigotimes_{A_{1}} \text {; } \\
& \stackrel{\epsilon^{n} \cdot J_{1} \bigotimes \cdots \bigotimes J_{n}}{\longrightarrow}, H_{1} \bigotimes_{A_{2}} \ldots \bigotimes_{A_{n}} H_{n} \bigotimes_{A_{1} ;}, \stackrel{\left(u^{(\mathcal{I})}\right)^{*}}{\longrightarrow} H_{\Sigma},
\end{aligned}
$$

where $J_{i}$ is the modular conjugation on $H_{i}=L^{2}\left(\mathcal{A}\left(S_{i}^{\top}\right)\right)$, and $\epsilon \in\{ \pm 1\}$ is the sign that appears in Lemma B.7 (the latter only depends on the conformal net $\mathcal{A}$, and is conjecturally equal to 1 ).

Our next task is to construct a canonical isomorphism (B.1) between $H_{\Sigma}$ and $L^{2} \mathcal{A}(S)$. This will occupy us for the remainder of this appendix. Recall that $\Sigma=S \times[0,1]$, and that $H_{\Sigma}$ is the associated Hilbert space, constructed in B.13.

Theorem B.17. There is a canonical isomorphism of $S \sqcup \bar{S}$-sectors

$$
w: L^{2} \mathcal{A}(S) \rightarrow H_{\Sigma}
$$

that intertwines the modular conjugation on $L^{2} \mathcal{A}(S)$ and the involution $J_{\Sigma}$ on $H_{\Sigma}$.

Proof. Recall from Theorem 1.20 that there is a canonical isomorphism $\mathcal{A}(S) \cong \bigoplus_{\lambda \in \Delta} \mathbf{B}\left(H_{\lambda}(S)\right)$. After applying the functor $L^{2}$, this becomes an isomorphism

$$
L^{2} \mathcal{A}(S) \cong \bigoplus_{\lambda} \mathbf{H S}\left(H_{\lambda}(S)\right) \cong \bigoplus_{\lambda} H_{\lambda}(S) \otimes \overline{H_{\lambda}(S)}
$$

where HS stands for the Hilbert space of Hilbert-Schmidt operators. Note that, by Lemma 1.17, the right hand side is non-canonically isomorphic to $\bigoplus_{\lambda} H_{\lambda}(S) \otimes H_{\bar{\lambda}}(\bar{S})$.

Let us simplify the notation and write $H_{\lambda}$ instead of $H_{\lambda}(S)$. We therefore have a canonical isomorphism $L^{2} \mathcal{A}(S) \cong \bigoplus_{\lambda} H_{\lambda} \otimes \overline{H_{\lambda}}$. We know from (1.18) that the $S \sqcup \bar{S}$ sectors $H_{\Sigma}$ and $L^{2} \mathcal{A}(S)$ are isomorphic. Therefore, in order to make the isomorphism canonical, it is enough to identify $\operatorname{hom}\left(H_{\lambda} \otimes \overline{H_{\lambda}}, H_{\Sigma}\right)$ and $\mathbb{C}$ for every $\lambda \in \Delta$. Once canonical isomorphisms

$$
\varpi_{\lambda}: \operatorname{hom}\left(H_{\lambda} \otimes \overline{H_{\lambda}}, H_{\Sigma}\right) \rightarrow \mathbb{C}
$$

are constructed, we may consider the isometries

$$
w_{\lambda}:=\frac{\varpi_{\lambda}^{-1}(1)}{\left\|\varpi_{\lambda}^{-1}(1)\right\|}: H_{\lambda} \otimes \overline{H_{\lambda}} \rightarrow H_{\Sigma} .
$$


Taking the sum over $\lambda$ will then give us the desired unitary isomorphism

$$
w:=\bigoplus_{\lambda} w_{\lambda}: L^{2} \mathcal{A}(S) \cong \bigoplus_{\lambda} H_{\lambda} \otimes \overline{H_{\lambda}} \longrightarrow H_{\Sigma} .
$$

As a first step towards (B.19), we construct an isomorphism

$$
\varpi_{I}=\varpi_{\lambda, I}: \operatorname{hom}\left(H_{\lambda} \otimes \overline{H_{\lambda}}, H_{\Sigma}\right) \rightarrow \mathbb{C}
$$

that depends on the choice of an interval $I \subset S$ and on the choice of local coordinates at the two endpoints of $I$. We will show later that this map is in fact independent of the choice of interval and of local coordinates. Let $I^{\prime}$ be the closure of $S \backslash I$, and let

$$
S_{I}:=\partial(I \times[0,1]) \quad S_{I^{\prime}}:=\partial\left(I^{\prime} \times[0,1]\right) \quad S_{I}^{+}:=\partial\left(I \times\left[\frac{1}{2}, 1\right]\right) \quad S_{I}^{-}:=\partial\left(I \times\left[0, \frac{1}{2}\right]\right)
$$

be as in the following picture:
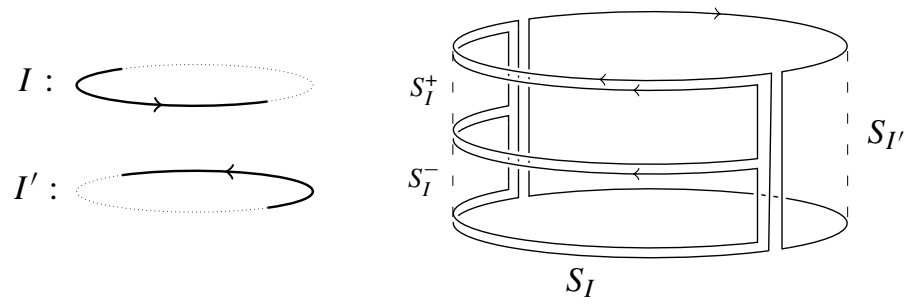

Recall that $\partial(I \times[0,1]) \top$ denotes the upper half of $S_{I}$, and let us write $\partial(I \times[0,1])_{\perp}$ for the corresponding lower half.

Let also $H_{I}:=H_{0}\left(S_{I}\right), H_{I}^{\prime}:=H_{0}\left(S_{I^{\prime}}\right), H_{I}^{+}:=H_{0}\left(S_{I}^{+}\right), H_{I}^{-}:=H_{0}\left(S_{I}^{-}\right)$be the correspdonding vacuum sectors. More precisely, we take $H_{I}$ to be the vacuum sector associated to the circle $S_{I}$, its upper half $\partial(I \times[0,1])_{\top}$, and the involution $j:(x, t) \mapsto$ $(x, 1-t)$. Similarly, we let $H_{I}^{\prime}$ be the vacuum sector associated to $S_{I^{\prime}}$, its upper half, and $j$. The vacuum sector $H_{I}^{+} \in \operatorname{Sect}_{S_{I}^{+}}(\mathcal{A})$ is chosen arbitrarily in its isomorphism class. Finally, we take $H_{I}^{-}:=j^{*}\left(H_{I}^{+}\right) \cdot{ }^{10}$ Let also

$$
\begin{gathered}
A:=\mathcal{A}\left(I^{\prime} \times\{0,1\}\right), \quad B:=\mathcal{A}(I \times\{0,1\}), \quad C:=\mathcal{A}(\partial I \times[0,1]), \\
B_{0}:=\mathcal{A}(I \times\{0\}), \quad B_{1}:=\mathcal{A}(I \times\{1\}), \quad B_{1 / 2}:=\mathcal{A}(I \times\{1 / 2\}), \\
D_{0}:=\mathcal{A}\left(\partial(I \times[0,1])_{\perp}\right), \quad D_{1}:=\mathcal{A}(\partial(I \times[0,1]) \top)
\end{gathered}
$$

with orientations as indicated here:

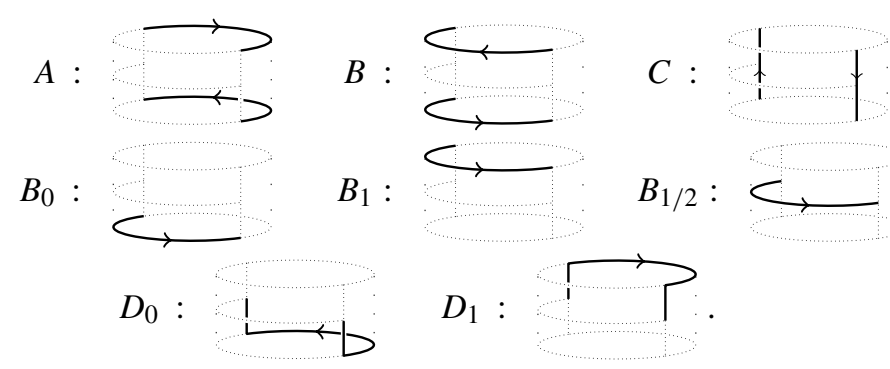

$\overline{10}$ The functor $j^{*}: \operatorname{Sect}_{S_{I}^{+}}(\mathcal{A}) \rightarrow \operatorname{Sect}_{S_{I}^{-}}(\mathcal{A})$ is defined in [8, Eq. (1.12)]. 
Finally, let us denote by $D$ the algebra $\mathcal{A}\left(\left[0, \frac{1}{2}\right] \cup I^{\prime} \cup\left[0, \frac{1}{2}\right]\right)$, which we identify with $D_{1}$ in the obvious way. With all those preliminaries in place, we can now define the map (B.21) as the composite of the following isomorphisms:

$$
\begin{aligned}
\varpi_{I}: \operatorname{hom}\left(H_{\lambda} \otimes \overline{H_{\lambda}}, H_{\Sigma}\right) & \rightarrow \operatorname{hom}_{A \vee B}\left(H_{\lambda} \otimes \overline{H_{\lambda}}, H_{I} \otimes_{C} H_{I}^{\prime}\right) \\
& \rightarrow \operatorname{hom}_{A \vee C}\left(\left(H_{\lambda} \otimes \overline{H_{\lambda}}\right) \bigotimes_{B} \overline{H_{I}}, H_{I}^{\prime}\right) \\
& \rightarrow \operatorname{hom}_{A \vee C}\left(\left(H_{\lambda} \otimes \overline{H_{\lambda}}\right) \bigotimes_{B} H_{I}, H_{I}^{\prime}\right) \\
& \rightarrow \operatorname{hom}_{A \vee C}\left(H_{\lambda} \otimes_{B_{0}} H_{I} \bigotimes_{B_{1}} \overline{H_{\lambda}}, H_{I}^{\prime}\right) \\
& \rightarrow \operatorname{hom}_{A \vee C}\left(H_{\lambda} \otimes_{B_{0}} H_{I}^{-} \otimes_{B_{1 / 2}} H_{I}^{+} \otimes_{B_{1}} \overline{H_{\lambda}}, H_{I}^{\prime}\right) \\
& =\operatorname{hom}_{D_{0} \vee D_{1}}\left(H_{\lambda} \otimes_{B_{0}} H_{I}^{-} \bigotimes_{B_{1 / 2}} H_{I}^{+} \bigotimes_{B_{1}} \overline{H_{\lambda}}, H_{I}^{\prime}\right) \\
& =\operatorname{hom}_{D, D}\left(H_{\lambda} \otimes_{B_{0}} H_{I}^{-} \bigotimes_{B_{1 / 2}} H_{I}^{+} \bigotimes_{B_{1}} \overline{H_{\lambda}}, L^{2}(D)\right) \\
& \rightarrow \mathbb{C} .
\end{aligned}
$$

The first map is the isomorphism (B.14) associated to the $c$-cover $\left\{I, I^{\prime}\right\}$ of $S$. The second map is a duality isomorphism, using that $\mathcal{A}$ has finite index. The third map is the modular conjugation of $H_{I}=L^{2} \mathcal{A}(\partial(I \times[0,1]) \top)$. The fourth map is an instance Lemma A.4. The fifth map is the counit of the duality between $H_{I}^{-}$and $H_{I}^{+}$. Finally, the sixth and last map sends the counit of the duality between $H_{\lambda} \otimes_{B_{0}} H_{I}^{-}$and $H_{I}^{+} \otimes_{B_{1}} \overline{H_{\lambda}}$ to the complex number $1 \in \mathbb{C}$.

At this point, we can check that (B.20) intertwines the modular conjugation $J$ on $L^{2} \mathcal{A}(S)$ and the involution $J_{\Sigma}$ on $H_{\Sigma}$. Note that the restriction of $J$ to $H_{\lambda} \otimes \overline{H_{\lambda}}$ is the map that exchanges the two factors. We need to show that $\varpi_{\lambda}^{-1}(1): H_{\lambda} \otimes \bar{H}_{\lambda} \rightarrow H_{\Sigma}$ intertwines that involution with $J_{\Sigma}$. In other words, we need to show that $\varpi_{\lambda}^{-1}(1)$ is invariant under the natural involution on $\operatorname{hom}\left(H_{\lambda} \otimes \overline{H_{\lambda}}, H_{\Sigma}\right)$. All the spaces in (B.22) are equipped with their own antilinear involution, and all the maps are compatible with the involutions. The invariance of $\varpi_{\lambda}^{-1}(1)$ then follows from the invariance of $1 \in \mathbb{C}$ under complex conjugation.

Given two intervals $I_{1}, I_{2} \subset S$, each one with local coordinates at their boundary, we still need to show that $\varpi_{I_{1}}=\varpi_{I_{2}}$. Without loss of generality, we may assume that $I_{1} \subset I_{2}$, that the two intervals share a common boundary point $p$, and that the local coordinates agree at that point. Let $\varphi \in \operatorname{Diff}_{+}(S)$ be a diffeomorphism that sends $I_{1}$ to $I_{2}$ and that is compatible with the local coordinates. In particular, $\varphi$ fixes a neighborhood of the point $p$. Pick an interval $J$ that contains $\operatorname{supp}(\varphi)$ in its interior and that misses the point $p$, and let $v \in \mathcal{A}(J)$ be a unitary that implements $\varphi$, that is, such that $\operatorname{Ad}(v)=\mathcal{A}(\varphi)$.

Let $H_{1}:=H_{I_{1}}, H_{1}^{\prime}:=H_{I_{1}}^{\prime}, H_{1}^{+}:=H_{I_{1}}^{+}, H_{1}^{-}:=H_{I_{1}}^{-}$be the Hilbert spaces that enter in the definition of $\varpi_{I_{1}}$, and let $\mathrm{H}_{2}, \mathrm{H}_{2}^{\prime}, \mathrm{H}_{2}^{+}, \mathrm{H}_{2}^{-}$be the corresponding Hilbert spaces for $\varpi_{I_{2}}$. By evaluating $L^{2}(\mathcal{A}(-))$ on the diffeomorphisms that $\varphi$ induces between the upper halves of the appropriate circles, we get unitary isomorphisms $a: H_{1} \rightarrow H_{2}$ and $a^{\prime}: H_{1}^{\prime} \rightarrow H_{2}^{\prime}$. Let $b^{+}: H_{1}^{+} \rightarrow \varphi^{*}\left(H_{2}^{+}\right)$be an arbitrary $S_{I_{1}}^{+}$-sector isomorphism, and let $b^{-}:=j^{*}\left(b^{+}\right): H_{1}^{-} \rightarrow \varphi^{*}\left(H_{2}^{-}\right)$. Finally, let $c: H_{\lambda} \rightarrow H_{\lambda}$ and $\bar{c}: \overline{H_{\lambda}} \rightarrow \overline{H_{\lambda}}$ be the maps induced by multiplication by $v$. We then get a diagram 


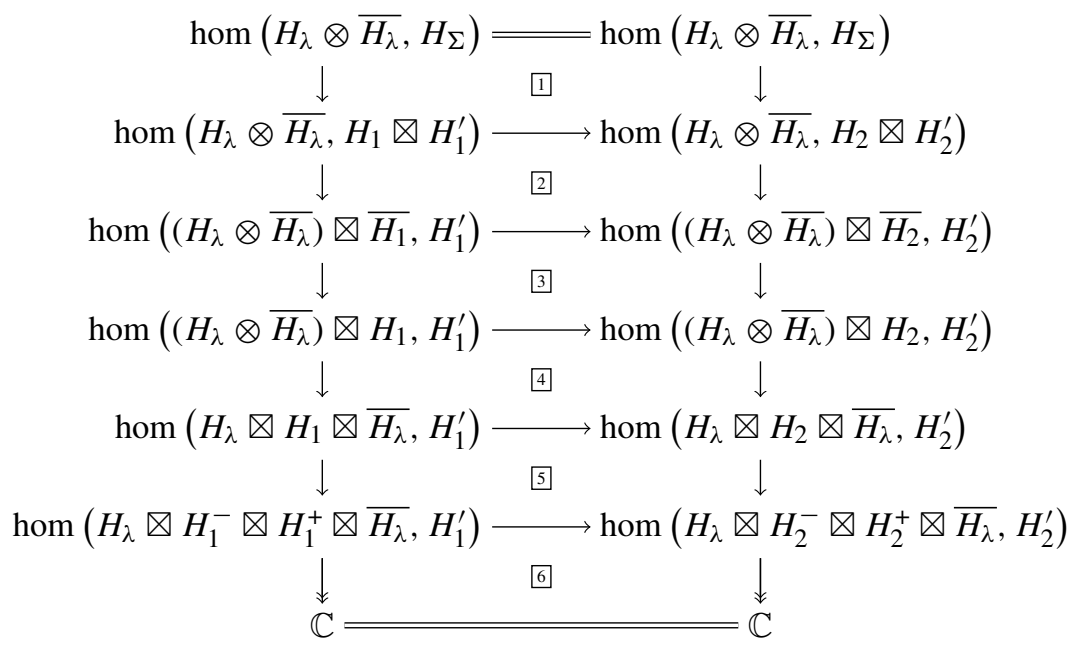

where various subscripts are left implicit, the vertical arrows compose to $\varpi_{I_{1}}$ and $\varpi_{I_{2}}$, and the horizontal arrows are induced by $a, a^{\prime}, b^{+}, b^{-}, c, \bar{c}$. The squares $2,3, \ldots, 6$ are easily seen to commute. Finally, the commutativity of 1 is the content of Lemma B.23.

Recall that $H_{1}=H_{I_{1}}, H_{2}=H_{I_{2}}, H_{1}^{\prime}=H_{I_{1}}^{\prime}, H_{2}^{\prime}=H_{I_{2}}^{\prime}$ are the vacuum sectors associated to the boundaries of $I_{1} \times[0,1], I_{2} \times[0,1], I_{1}^{\prime} \times[0,1]$, and $I_{2}^{\prime} \times[0,1]$.

Lemma B.23. Let $H_{\lambda}, H_{\Sigma}, H_{I_{1}}, H_{I_{1}}^{\prime}, H_{I_{2}}, H_{I_{2}}^{\prime}, a, a^{\prime}, c, \bar{c}$ be as above, and let $u_{1}$ : $H_{\Sigma} \rightarrow, H_{I_{1}} \otimes H_{I_{1}}^{\prime} \bigotimes_{j}$; and $u_{2}: H_{\Sigma} \rightarrow, H_{I_{2}} \otimes H_{I_{2}}^{\prime} \otimes$; be instances of (B.14). Then the triangle

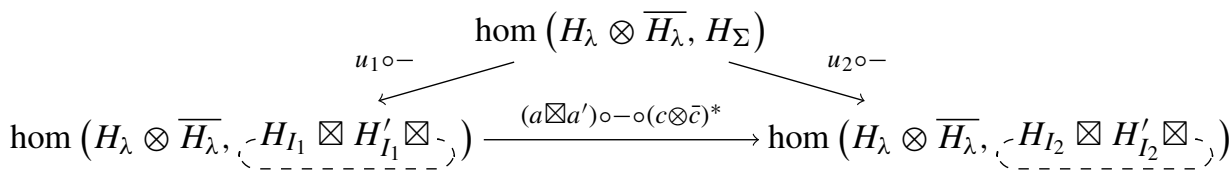
is commutative, where the hom are taken in the category of $S \sqcup \bar{S}$-sectors.

Proof. Given a morphism of $S \sqcup \bar{S}$-sectors $f: H_{\lambda} \otimes \overline{H_{\lambda}} \rightarrow H_{\Sigma}$, we need to show that $\left(a \otimes a^{\prime}\right) \circ u_{1} \circ f \circ(c \otimes \bar{c})^{*}=u_{2} \circ f$. Recall that $c$ is the operator of multiplication by the unitary $v$, defined above. The operator

$$
c \otimes \bar{c}: H_{\lambda} \otimes \overline{H_{\lambda}} \rightarrow H_{\lambda} \otimes \overline{H_{\lambda}}
$$

is therefore given by multiplication by $v \otimes \bar{v}$. Since $u_{1} \circ f$ commutes with the action of that element, we have

$$
\left(a \otimes a^{\prime}\right) \circ u_{1} \circ f \circ(c \otimes \bar{c})^{*}=\left(a \otimes a^{\prime}\right) \circ(v \otimes \bar{v})^{*} \circ u_{1} \circ f,
$$

and so it is enough to show that $\left(a \otimes a^{\prime}\right)(v \otimes \bar{v})^{*} u_{1}=u_{2}$. In other words, we need to show that the following triangle commutes:

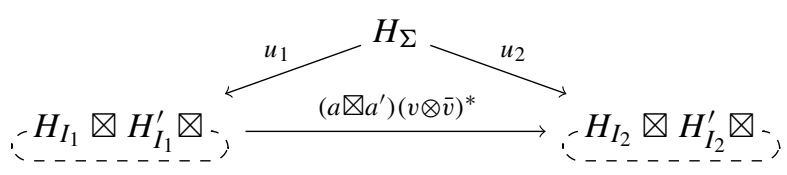


Recall that $I_{1}$ is contained in $I_{2}$, and that $p$ is their common boundary point. Recall that $\varphi$ is a diffeomorphism that satisfies $\varphi\left(I_{1}\right)=I_{2}$, and that it is the identity in a neighborhood of $p$. Finally, recall that $\operatorname{Ad}(v)=\mathcal{A}(\varphi)$. Let $q \in I_{2}^{\prime}$ be such that the interval $K:=[q, p] \subset S$ is disjoint from $\operatorname{supp}(\varphi)$, and let $J_{1}$ and $J_{2}$ be the closures of $I_{1}^{\prime} \backslash K$ and $I_{2}^{\prime} \backslash K$.
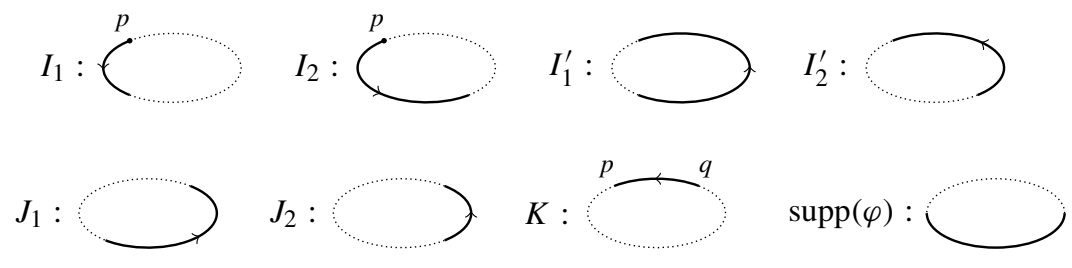

Chose a local coordinate at the point $q$, and let $H_{J_{1}}, H_{J_{2}}, H_{K}$ be the vacuum sectors associated to the boundaries of $J_{1} \times[0,1], J_{2} \times[0,1], K \times[0,1]$, their upper halves, and the involution $j$. We then have the following two commutative triangles

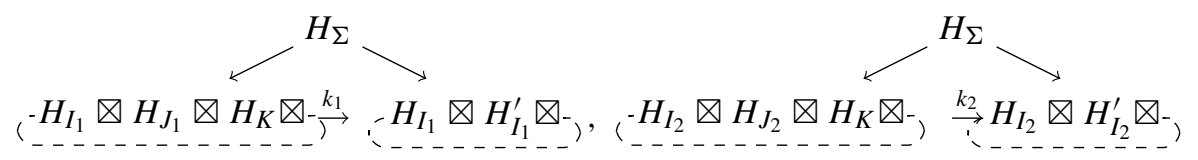

where the horizontal maps $k_{1}$ and $k_{2}$ are induced by (B.5), and the maps from $H_{\Sigma}$ are instances of (B.14). There is also a commutative square

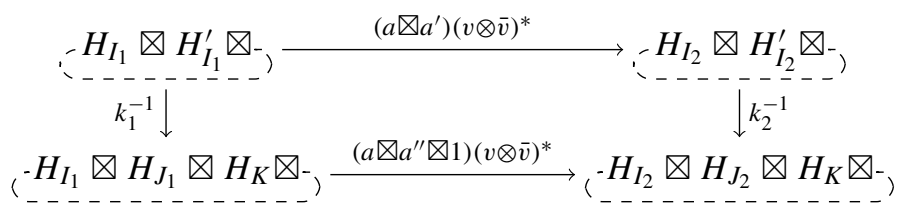

where $a^{\prime \prime}: H_{J_{1}} \rightarrow H_{J_{2}}$ is the value of $L^{2}(\mathcal{A}(-))$ on the diffeomorphism that $\varphi$ induces from the upper half of $\partial\left(J_{1} \times[0,1]\right)$ to the upper half of $\partial\left(J_{2} \times[0,1]\right)$. In view of (B.25) and (B.26), the commutativity of (B.24) is equivalent to that of

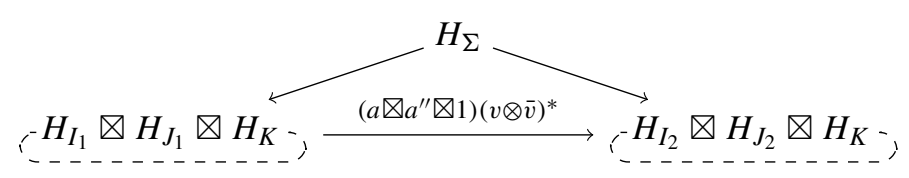

where the maps down from $H_{\Sigma}$ are again instances of (B.14). Consider now the following two commutative triangles

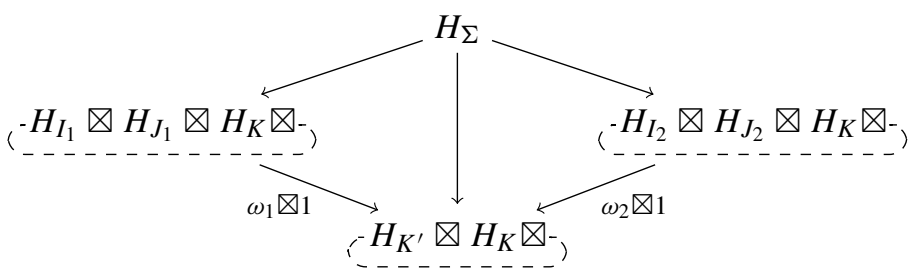


where $\omega_{1}: H_{I_{1}} \otimes H_{J_{1}} \rightarrow H_{K^{\prime}}$ and $\omega_{2}: H_{I_{2}} \otimes H_{J_{2}} \rightarrow H_{K^{\prime}}$ are instances of (B.5), and the maps from $H_{\Sigma}$ are as in (B.14). Using (B.28), we can further reduce (B.27) to the commutativity of this triangle:

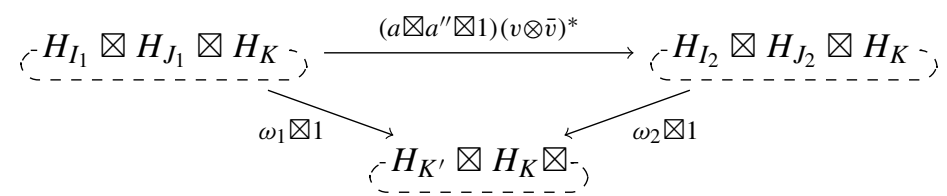

Recall that the support of $\varphi$ is disjoint from $K$. It follows that $v \in \mathcal{A}\left(K^{\prime}\right)$, and that the operator $v \otimes \bar{v}$ only acts on $H_{I_{1}} \otimes H_{J_{1}}$. The commutativity of (B.29) therefore boils down to the commutativity of this diagram:

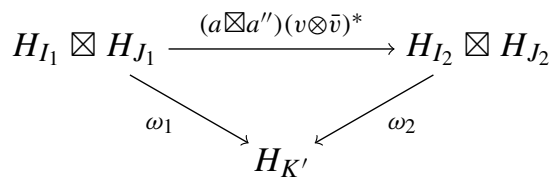

Write

$$
\begin{gathered}
\varphi_{I}: \partial\left(I_{1} \times[0,1]\right)_{\top} \rightarrow \partial\left(I_{2} \times[0,1]\right)_{\top} \quad \varphi_{J}: \partial\left(J_{1} \times[0,1]\right)_{\top} \rightarrow \partial\left(J_{2} \times[0,1]\right)_{\top} \\
\varphi_{K}: \partial\left(K^{\prime} \times[0,1]\right) \top \rightarrow \partial\left(K^{\prime} \times[0,1]\right)_{\top}
\end{gathered}
$$

for the maps induced by $\varphi$, and recall that $a=L^{2} \mathcal{A}\left(\varphi_{I}\right)$ and $a^{\prime \prime}=L^{2} \mathcal{A}\left(\varphi_{J}\right)$. Pick

$f_{I}: \partial\left(I_{1} \times[0,1]\right)_{\top} \rightarrow S_{\top}^{1} \quad f_{J}: \partial\left(J_{1} \times[0,1]\right)_{\top} \rightarrow S_{\top}^{1} \quad f_{K}: \partial\left(K^{\prime} \times[0,1]\right)_{\top} \rightarrow S_{\top}^{1}$

with the same properties as the maps $f_{1}, f_{2}, f_{3}$ that enter (B.6), and let

$g_{I}: \partial\left(I_{2} \times[0,1]\right)_{\top} \rightarrow S_{\top}^{1} \quad g_{J}: \partial\left(J_{2} \times[0,1]\right)_{\top} \rightarrow S_{\top}^{1} \quad g_{K}: \partial\left(K^{\prime} \times[0,1]\right)_{\top} \rightarrow S_{\top}^{1}$

be given by $g_{\alpha}=f_{\alpha} \circ \varphi_{\alpha}^{-1}, \alpha \in\{I, J, K\}$. By definition, we have

$$
\begin{aligned}
& \omega_{1}=\left(L^{2} \mathcal{A}\left(f_{K}\right)^{-1}\right)\left(1 \otimes v_{\vdash} v_{\top}^{-1}\right)\left(L^{2} \mathcal{A}\left(f_{I}\right) \otimes L^{2} \mathcal{A}\left(f_{J}\right)\right) \\
& \omega_{2}=\left(L^{2} \mathcal{A}\left(g_{K}\right)^{-1}\right)\left(1 \otimes v_{\vdash} v_{\top}^{-1}\right)\left(L^{2} \mathcal{A}\left(g_{I}\right) \otimes L^{2} \mathcal{A}\left(g_{J}\right)\right) .
\end{aligned}
$$

For $w:=\mathcal{A}\left(f_{K}\right)(v)$, it follows from $\operatorname{Ad}(v)=\mathcal{A}\left(\varphi_{K}\right)$ that $\operatorname{Ad}(w)=\mathcal{A}\left(f_{K} \circ g_{K}^{-1}\right)$. Writing $H_{0}$ for $L^{2} \mathcal{A}\left(S_{\top}^{1}\right)$, the triangle (B.30) can then be decomposed as

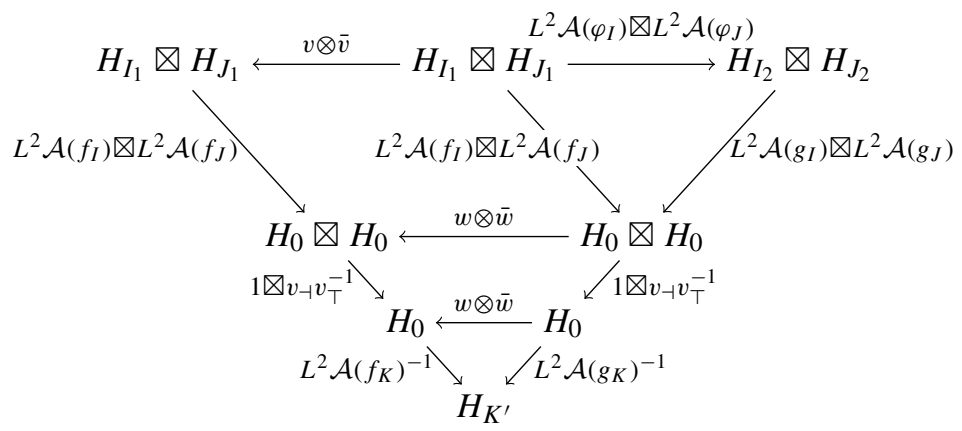

and it is now clear that each one of the pieces commutes. 


\section{References}

1. Andersen, J.E., Ueno, K.: Abelian conformal field theory and determinant bundles. Int. J. Math. 18(8), 919-993 (2007)

2. Andersen, J.E., Ueno, K.: Geometric construction of modular functors from conformal field theory. J. Knot Theory Ramifications 16(2), 127-202 (2007)

3. Andersen, J.E., Ueno, K.: Modular functors are determined by their genus zero data. Quantum Topol. 3(34), 255-291 (2012)

4. Andersen, J.E., Ueno, K.: Construction of the Reshetikhin-Turaev TQFT from conformal field theory. Invent. Math. 201(2), 519-559 (2015)

5. Axelrod, S., Della Pietra, S., Witten, E.: Geometric quantization of Chern-Simons gauge theory. J. Differ. Geom. 33(3), 787-902 (1991)

6. Bakalov, B., Kirillov, A. Jr.: Lectures on Tensor Categories and Modular Functors, University Lecture Series, vol. 21. American Mathematical Society, Providence (2001)

7. Bartels, A., Douglas, C.L., Henriques, A.: Dualizability and index of subfactors. Quantum Topol., 5, 289-345 (2014). arXiv:1110.5671

8. Bartels, A., Douglas, C.L., Henriques, A.: Conformal nets I: coordinate-free nets. Int. Math. Res. Not., 13, 4975-5052 (2015). arXiv:1302.2604v2

9. Bartels, A., Douglas, C.L., Henriques, A.: Conformal nets V: dualizability (2017) (in preparation)

10. Beauville, A., Laszlo, Y.: Conformal blocks and generalized theta functions. Commun. Math. Phys. 164(2), 385-419 (1994)

11. Belavin, A.A., Polyakov, A.M., Zamolodchikov, A.B.: Infinite conformal symmetry in two-dimensional quantum field theory. Nuclear Phys. B 241(2), 333-380 (1984)

12. Beliakova, A., Blanchet, C.: Modular categories of types $B, C$ and D. Comment. Math. Helv. 76(3), 467500 (2001)

13. Bisch, D.: Bimodules, higher relative commutants and the fusion algebra associated to a subfactor. In: Operator Algebras and Their Applications (Waterloo, ON, 1994/1995), Fields Institute Communications, vol. 13, pp. 13-63. American Mathematical Society, Providence, (1997)

14. Blanchet, C.: Hecke algebras, modular categories and 3-manifolds quantum invariants. Topology 39(1), 193-223 (2000)

15. Brunetti, R., Guido, D., Longo, R.: Modular structure and duality in conformal quantum field theory. Commun. Math. Phys. 156(1), 201-219 (1993)

16. Carpi, S., Conti, R., Hillier, R., Weiner, M.: Representations of conformal nets, universal C*-algebras and K-theory. Commun. Math. Phys. 320(1), 275-300 (2013)

17. Carpi, S., Kawahigashi, Y., Longo, R., Weiner, M.: From Vertex Operator Algebras to Conformal Nets and Back. arXiv:1503.01260 (2015)

18. Connes, A.: Noncommutative Geometry. Academic Press Inc., San Diego (1994)

19. Dong, C., Lin, X.: Unitary vertex operator algebras. J. Algebra 397, 252-277 (2014)

20. Faltings, G.: A proof for the Verlinde formula. J. Algebr. Geom. 3(2), 347-374 (1994)

21. Frenkel, E., Ben-Zvi, D.: Vertex algebras and algebraic curves. In: Mathematical Surveys and Monographs, vol. 88, 2nd edn. American Mathematical Society, Providence (2004)

22. Friedan, D., Shenker, S.: The analytic geometry of two-dimensional conformal field theory. Nuclear Phys. B, 281(3-4), 509-545 (1987)

23. Gabbiani, F., Fröhlich, J.: Operator algebras and conformal field theory. Commun. Math. Phys. 155(3), 569-640 (1993)

24. Hitchin, N.J.: Flat connections and geometric quantization. Commun. Math. Phys. 131(2), 347-380 (1990)

25. Huang, Y.-Z., Lepowsky, J.: Tensor categories and the mathematics of rational and logarithmic conformal field theory. J. Phys. A 46, 494009 (2013)

26. Kawahigashi, Y., Longo, R., Müger, M.: Multi-interval subfactors and modularity of representations in conformal field theory. Commun. Math. Phys. 219(3), 631-669 (2001)

27. Kumar, S., Narasimhan, M.S., Ramanathan, A.: Infinite Grassmannians and moduli spaces of $G$ bundles. Math. Ann. 300(1), 41-75 (1994)

28. Laszlo, Y.: Hitchin's and WZW connections are the same. J. Differ. Geom. 49(3), 547-576 (1998)

29. Longo, R.: Lectures on Conformal Nets II. http://www.mat.uniroma2.it/ longo/Lecture\%20Notes.html (2008)

30. Looijenga, E.: Unitarity of SL(2)-conformal blocks in genus zero. J. Geom. Phys. 59(5), 654-662 (2009)

31. Moore, G., Seiberg, N.: Classical and quantum conformal field theory. Commun. Math. Phys. 123(2), 177254 (1989)

32. Moore, G., Seiberg, N.: Naturality in conformal field theory. Nuclear Phys. B 313(1), 16-40 (1989)

33. Müger, M.: From subfactors to categories and topology. II. The quantum double of tensor categories and subfactors. J. Pure Appl. Algebra 180(1-2), 159-219 (2003)

34. Posthuma, H.: The Heisenberg group and conformal field theory. Q. J. Math. 63(2), 423-465 (2012) 
35. Ramadas, T.R.: The "Harder-Narasimhan trace" and unitarity of the KZ/Hitchin connection: genus 0 . Ann. Math. (2) 169(1), 1-39 (2009)

36. Rehren K.-H.: Braid group statistics and their superselection rules. In: The Algebraic Theory of Superselection Sectors (Palermo, 1989), pp. 333-355. World Scientific Publishing, River Edge (1990)

37. Rowell, E.C.: From quantum groups to unitary modular tensor categories. In: Representations of Algebraic Groups, Quantum Groups, and Lie Algebras, Contemporary Mathematics, vol. 413, pp. 215-230. American Mathematical Society, Providence (2006)

38. Sauvageot, J.-L.: Sur le produit tensoriel relatif d'espaces de Hilbert. J. Oper. Theory 9(2), 237-252 (1983)

39. Segal, G.: The definition of conformal field theory. In: Topology, geometry and quantum field theory, volume 308 of London Mathematical Society Lecture Note Serie, pp. 421-577. Cambridge University Press, Cambridge (2004)

40. Teleman, C.: Borel-Weil-Bott theory on the moduli stack of $G$-bundles over a curve. Invent. Math. 134(1), 1-57 (1998)

41. Tsuchiya, A., Ueno, K., Yamada, Y.: Conformal field theory on universal family of stable curves with gauge symmetries. In: Integrable Systems in Quantum Field Theory and Statistical Mechanics, vol. 19 Advanced Studies in Pure Mathematics, pp. 459-566. Academic Press, Boston (1989)

42. Wassermann, A.: Operator algebras and conformal field theory. III. Fusion of positive energy representations of $\mathrm{LSU}(N)$ using bounded operators. Invent. Math. 133(3), 467-538 (1998)

43. Wenzl, H.: $C^{*}$ tensor categories from quantum groups. J. Am. Math. Soc. 11(2), 261-282 (1998)

44. Xu, F.: Jones-Wassermann subfactors for disconnected intervals. Commun. Contemp. Math. 2(3), 307$347(2000)$

Communicated by Y. Kawahigashi 